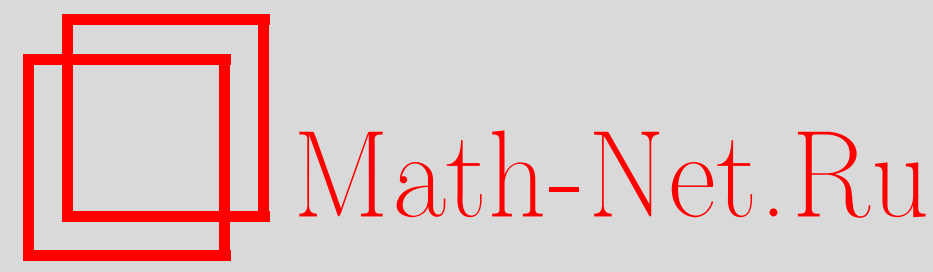

Ю. Н. Дрожжинов, Б. И. Завьялов, Тауберовы теоремы для обобщенных функций со значениями в банаховых пространствах, Изв. РАН. Сер. матем., 2002, том 66, выпуск 4, 47-118

DOI: https://doi.org/10.4213/im395

Использование Общероссийского математического портала Math-Net.Ru подразумевает, что вы прочитали и согласны с пользовательским соглашением

http://www.mathnet.ru/rus/agreement

Параметры загрузки:

IP : 54.210 .77 .194

26 апреля 2023 г., 07:05:36 
УДК 517.5

Ю.Н. Дрожжинов, Б.И. Завьялов

\title{
Тауберовы теоремы для обобщенных функций со значениями в банаховых пространствах
}

\begin{abstract}
Сформулированы и доказаны тауберовы теоремы нового типа. В этих теоремах указаны достаточные условия, при которых обобщенная функция, принимаюшая априори значения в локально выпуклом топологическом пространстве, на самом деле, принимает значения в более узком, банаховом, пространстве. Эти условия формулируются в терминах "общих оценок класса" для стандартного усреднения этой обобщенной функции с фиксированным ядром из основного пространства.

Применение такого рода теорем, в частности, основано на том, что асимптотические и некоторые другие свойства рассматриваемых обобщенных функций можно описать в терминах принадлежности некоторым банаховым пространствам. Доказанные теоремы применяются для изучения асимптотических свойств решений задачи Коши для уравнения теплопроводности в классе медленно растущих обобщенных функций и к изучению банаховых пространств типа БесоваНикольского.

Библиографиия: 13 наименований.
\end{abstract}

\section{Введение}

Пусть $f(x) \in \mathscr{S}^{\prime}$, т.е. является обобщенной функцией медленного роста и

$$
L_{f}^{e}(x, y)=\left(f(\xi), \frac{1}{y} e\left(\frac{x-\xi}{y}\right)\right)
$$

- ее стандартное усреднение с некоторым ядром $e(x) \in \mathscr{S}$. Пусть $f(x)$ и $e(x)$ таковы, что

$$
\operatorname{supp} \tilde{f}(t) \subset[0,+\infty), \quad \widetilde{e}(t)=e^{-t}, \quad t \geqslant 0
$$

где волной обозначено преобразование Фурье. Тогда $L_{f}^{e}(x, y)$ - обычное преобразование Лапласа обобщенной функции $\tilde{f}(t)$. Имеет место следующее утверждение, которое является простой переформулировкой общей тауберовой теоремы, рассмотренной в работе [3].

Пусть вьполнены условия (0.2); тогда следуюшие два утверждения эквивалентны:

1) $f(x)$ квазиасимптотически ограничена в нуле относительно правильно меняющейся функции $\rho(k)$, т.е. $\frac{1}{\rho(k)}\left|\left(f\left(\frac{x}{k}\right), \varphi(x)\right)\right|<C_{\varphi}$ при $k>1$ и всех $\varphi \in \mathscr{S}$;

Работа выполнена при финансовой поддержке РФФИ (гранты № 02-01-00076, 00-15-96073).

(С) Ю. Н. Дрожжинов, Б. И. ЗАвьялов, 2002 
2) сушествуют $C$ и $b$ такие, что

$$
\frac{1}{\rho(k)}\left|L_{f}^{e}\left(\frac{x}{k}, \frac{y}{k}\right)\right| \leqslant \frac{C}{y^{b}}, \quad x^{2}+y^{2}=1, \quad y>0, \quad k>1 .
$$

Отметим, что если на $f$ наложено добавочное тауберово условие (например, $\tilde{f}$ есть положительная мера, как в теореме Харди-Литтлвуда, или $f(x+i y)$ имеет неотрищательную мнимую часть, как в общей тауберовой теореме работы [3]), то условие (0.3) может быть заменено на более простое условие

$$
\left|\frac{y}{\rho\left(\frac{1}{y}\right)} L_{f}^{e}(0, y)\right|<C, \quad 0<y<1 \text {. }
$$

В этой формулировке условия (0.2) выглядят весьма обременительными (они и только они содержат преобразование Фурье). Как будет показано в настоящей работе, они являются излишними.

На самом деле, эти и другие сходные проблемы могут быть рассмотрены с более общей позиции, где квазиасимптотические и другие свойства рассматриваемых функций описываются в терминах принадлежности некоторым банаховым пространствам. Пусть $f$ - обобщенная функция из $\mathscr{S}^{\prime}$ со значениями в банаховом пространстве $\mathbb{B}$, т.е. линейное непрерывное отображение из $\mathscr{S}$ в $\mathbb{B}(f \in \mathbb{L}(\mathscr{S} \rightarrow \mathbb{B}))$. Для ее стандартного усреднения с любым фиксированным ядром $\varphi \in \mathscr{S}$ справедлива оценка

$$
\sup _{0<y \leqslant 1}\left(y^{b}\left\|L_{f}^{\varphi}(x, y)\right\|_{\mathbb{B}}\right) \leqslant C(1+|x|)^{a}, \quad x \in \mathbb{R}
$$

называемая общей оценкой класса, для некоторых постоянных $a, b$ и $C$. Пусть $\mathbb{B}$ вложено в некоторое локально выпуклое топологическоепространство $\mathscr{L}$ и $f$ априори есть линейное непрерывное отображение из $\mathscr{S}$ в $\mathscr{L}$, т.е. $f \in \mathbb{L}(\mathscr{S} \rightarrow \mathscr{L})$. Тогда условие (0.5) можно рассматривать как необходимое условие принадлежности функции $f$ классу $\mathbb{L}(\mathscr{S} \rightarrow \mathbb{B})$. Представляет интерес выяснить, насколько это условие является достаточным. Настоящая работа, в основном, повящена этому. В частности, будет показано, что таким достаточным условием является оценка $(0.5)$, выполненная лишь для одной основной функции $\varphi$ такой, что $\int \varphi(\xi) d \xi \neq 0$. Отметим также, что пространство $\mathscr{S}$ можно заменить на другие пространства основных функций, такие как $\mathscr{D}, E^{\uparrow}, E^{\downarrow}$ (см. ниже).

Кроме того, для приложений весьма важно наряду с функционалом $\sup _{y}(\cdot)$ pacсматривать и другие функционалы. Для этой цели введем некий специальньй класс функционалов, которые назовем автомодельными. Он является естественным для данного круга задач и включает в себя стандартные функционалы типа норм пространств $L^{p}$ с весом.

Рассмотрим случай, когда в локально выпуклом пространстве $\mathscr{L}$ задано представление $\left\{T_{a}: \mathscr{L} \rightarrow \mathscr{L}, a \in \mathbb{R}^{1}\right\}$ группы сдвигов, относительно которой наше банахово пространство $\mathbb{B}$ инвариантно. Это позволит усилить некоторые основные теоремы. В частности, если потребовать ковариантность рассматриваемой функции, т.е.

$$
(f(\xi), \varphi(\xi-a))=T_{a}(f(\xi), \varphi(\xi)) \quad \forall \varphi \in \mathscr{S}
$$


то оценки типа (0.3) можно заменить оценками типа (0.4). Тем самым условие (0.6) можно рассматривать как тауберово условие.

Полученные теоремы применяются для исследования асимптотических свойств решений задачи Коши для уравнения теплопроводности и для изучения пространств типа Бесова-Никольского в $\mathbb{R}^{1}$. Пространствам такого типа посвящена весьма обширная литература (см., например, [12], [13] и цитируемую там библиографию). Наше рассмотрение предполагает несколько другой подход, который, как нам кажется, содержит ряд интересных моментов. Из их числа упомянем следуюшие. Во-первых, наши построения являются естественным применением доказанных в настоящей работе основных теорем. Во-вторых, при построении этих пространств традиционно в качестве базового пространства использовались пространства $L^{p}$. В рассматриваемом случае базовым пространством может быть функциональное пространство достаточно общего вида, при этом гладкость функций соответствуюших пространств определяется некоторым автомодельным функционалом. В-третьих, при построении этих пространств ранее использовались усреднения либо с одной специальной функцией (ядро Пуассона [13]), либо с семейством основных функций (см. [8]). Покажем, что достаточно рассматривать усреднения лишь с одной более или менее произвольной основной функцией.

В приложение мы вынесли несколько лемм, которые являются некоторыми вариантами классической теоремы Карлесона о короне. Их доказательство в основном тексте сильно перегрузило бы изложение и затруднило восприятие основных результатов.

\section{§ 1. Основные обозначения и определения}

Через $\mathscr{D}$ обозначаем пространство бесконечно дифференцируемых финитных функций. Точнее, $\mathscr{D}$ - локально выпуклое пространство, являющееся индуктивным пределом (по $a>0)$ пространств $\mathscr{D}_{a}$, состоящих из функций $\varphi \in C^{\infty}(\mathbb{R})$ таких, что $\operatorname{supp} \varphi \subset[-a, a]$, с топологией, задаваемой системой полунорм:

$$
\mathscr{P}_{N}[\varphi]=\max _{0 \leqslant j \leqslant N} \sup _{-\infty<\xi<\infty}\left(1+\xi^{2}\right)^{\frac{N}{2}}\left|\varphi^{(j)}(\xi)\right|, \quad N=0,1, \ldots
$$

Аналогично, $\mathscr{S}$ - пространство быстроубывающих вместе со всеми производными функщий $\varphi(\xi)$ с топологией, задаваемой счетной системой полунорм (1.1).

Соответственно, $\mathscr{D}^{\prime}$ и $\mathscr{S}^{\prime}$ - сопряженные пространства (пространство обобщенных функций и пространство обобщенных функций медленного роста).

Введем пространство $E_{a}$, состоящее из бесконечно дифференцируемых на всей оси функций $\varphi(\xi)$, для которых

$$
P_{a, N}[\varphi]=\max _{0 \leqslant j \leqslant N} \sup _{-\infty<\xi<\infty} e^{a|\xi|}(1+|\xi|)^{N}\left|\varphi^{(j)}(\xi)\right|<\infty, \quad N=0,1, \ldots
$$

Эта система полунорм задает в $E_{a}$ топологию. При любом $a>0$ это пространство изоморфно пространству $\mathscr{S}$, а при $a=0$ оно совпадает с $\mathscr{S}$. Функции $\varphi(\xi)$ принадлежат $E_{a}$ тогда и только тогда, когда $e^{a \xi} \varphi(\xi)$ и $e^{-a \xi} \varphi(\xi)$ принадлежат $\mathscr{S}$. Через $E^{\uparrow}$ обозначаем индуктивный предел пространств $E_{a}$ по $a>0$, так что 
$E^{\uparrow}=\bigcup_{a>0} E_{a}$. Как обычно, $E^{\uparrow^{\prime}}-$ пространство линейных непрерывных функционалов на $E^{\uparrow}$.

Через $E^{\downarrow}$ обозначаем проективный предел пространств $E_{a}$ по $a>0$, так что $E^{\downarrow}=\bigcap_{a>0} E_{a}$, и $E^{\downarrow^{\prime}}-$ пространство линейных непрерывных функционалов на $E^{\downarrow} . Я_{\text {сно }}{ }^{1}$, что $\mathscr{D} \subset E^{\downarrow} \subset E^{\uparrow} \subset \mathscr{S}$.

Совокупность основных функций из $\mathscr{D}_{a}, \mathscr{S}$ или $E_{a}$, все моменты которых вплоть до $n$-го равны нулю, обозначаем $\stackrel{n}{\mathscr{D}} a, \stackrel{n}{\mathscr{S}}$ и $\stackrel{n}{E}_{a}$. Условимся считать, что $\stackrel{n}{\mathscr{D}}=\mathscr{D}$ при $n<0$ (соответственно, $\left.\stackrel{n}{\mathscr{S}}=\mathscr{S}, \stackrel{n}{E_{a}}=E_{a}\right)$. Таким образом,

$$
\stackrel{n}{\mathscr{D}}_{a}=\left\{\varphi(\xi) \in \mathscr{D}_{a}: \int \xi^{j} \varphi(\xi) d \xi=0, \quad j=0,1, \ldots, n\right\} .
$$

Например, $\varphi(\xi) \in \stackrel{n}{\mathscr{D}} \backslash \stackrel{n+1}{\mathscr{D}}$ означает, что

$$
\varphi(\xi) \in \mathscr{D}, \quad \int \xi^{j} \varphi(\xi) d \xi=0, \quad j=0,1, \ldots, n ; \quad \int \xi^{n+1} \varphi(\xi) d \xi \neq 0
$$

В частности, $\varphi(\xi) \in \mathscr{D} \backslash \stackrel{0}{\mathscr{D}}$ означает, что $\varphi(\xi) \in \mathscr{D}$ и $\int \varphi(\xi) d \xi \neq 0$.

Преобразование Фурье осушествляет изоморфизм пространства $E_{a}$ на пространство $\widetilde{E}_{a}$, состояшее из аналитических в полосе

$$
\Pi_{-a}^{a}=\{z \in \mathbb{C}: z=x+i y,|x|<\infty,-a<y<a\}
$$

функций $\widetilde{\varphi}(z)$, непрерывных вместе со всеми производными в замкнутой полосе $\bar{\Pi}_{-a}^{a}$ и удовлетворяющих оценке

$$
\left|\left(\frac{d}{d z}\right)^{\ell} \widetilde{\varphi}(z)\right| \leqslant \frac{c_{\ell, m}}{1+|z|^{m}}, \quad z \in \bar{\Pi}_{-a}^{a}, \quad m=1,2, \ldots, \quad \ell=0,1, \ldots
$$

Другими словами, $F\left[E_{a}\right]=\widetilde{E}_{a}$.

Напомним также, что $F[\mathscr{S}]=\mathscr{S}, F[\mathscr{D}]=Z, F\left[\mathscr{D}_{a}\right]=Z_{a}$. Пространство $Z_{a}$ состоит из целых аналитических функций. Топология в нем определяется системой полунорм:

$$
\widetilde{\mathscr{P}}_{a, N}[\widetilde{\varphi}(z)]=\sup _{|x|<\infty}(1+|z|)^{N} e^{a|y|}|\widetilde{\varphi}(z)|, \quad z=x+i y, \quad N=0,1, \ldots
$$

Пусть $\underset{n}{Z}=F[\stackrel{n}{\mathscr{D}}]$, так что для любого $a>0$

$$
Z_{n}=\left\{\widetilde{\varphi}(z) \in Z_{a}:\left.\left(\frac{d}{d z}\right)^{j} \widetilde{\varphi}(z)\right|_{z=0}=0, j=0,1, \ldots, n\right\}
$$

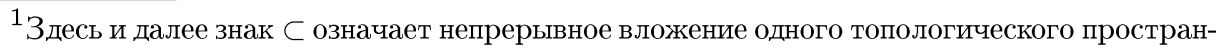
ства в другое.
} 


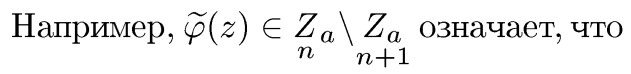

$$
\widetilde{\varphi}(z)=z^{n+1} \widetilde{\varphi}_{1}(z), \quad \widetilde{\varphi}_{1} \in Z_{a}, \quad \widetilde{\varphi}_{1}(0) \neq 0
$$

Подробнее о свойствах пространства $Z$ см.[2].

Через $A_{Q}\left(\Pi_{-a}^{a}\right)$, где $-\infty<Q<+\infty$, будем обозначать пространство аналитических в полосе $\Pi_{-a}^{a}$ функций $\widetilde{\varphi}(z)$ таких, что

$$
\|\widetilde{\varphi}(z)\|_{A_{Q}\left(\Pi_{-a}^{a}\right)}=\sup _{z \in \Pi_{-a}^{a}}(1+|z|)^{Q}|\widetilde{\varphi}(z)|<\infty .
$$

Через $A\left(\bar{\Pi}_{-a}^{a}\right)$ обозначается множество аналитических в полосе $\Pi_{-a}^{a}$ фуннкцй $\widetilde{\varphi}(z)$, непрерывных вместе со всеми производными вплоть до границы, причем для любого $j=0,1, \ldots$ существуют $Q_{j}$ такие, что

$$
\widetilde{\varphi}^{(j)}(z) \in A_{Q_{j}}\left(\Pi_{-a}^{a}\right)
$$

Отметим, что функции из $A\left(\bar{\Pi}_{-a}^{a}\right)$ при любом $a>0$ являются мультипликаторами в $\widetilde{E}_{a}$.

Всюду далее $\chi_{[a, b]}(\xi)$ - характеристическая функция отрезка $[a, b]$,

$$
\chi_{[a, b]}(\xi)=\Theta(b-\xi)-\Theta(a-\xi)
$$

где $\Theta(\xi)$ - функция Хевисайда, равная нулю при $\xi<0$ и еденице при $\xi>0$.

Пространство $C_{0}(\mathbb{R})$ - банахово пространство непрерывных функций, стремящихся к нулю на бесконечности, с нормой $\|\varphi(\xi)\|=\sup _{\xi}|\varphi(\xi)|$. Будем также пользоваться пространством $C_{0}^{\infty}(\mathbb{R})$, состоящим из бесконечно дифференцируемых функций $\varphi(\xi)$, которые вместе со всеми своими производными стремятся к нулю при $|\xi| \rightarrow \infty$. Топология в нем задается системой полунорм:

$$
Q_{N}[\varphi]=\max _{0 \leqslant j \leqslant N} \sup _{\xi}\left|\varphi^{(j)}(\xi)\right|, \quad N=0,1, \ldots
$$

Пусть $\mathscr{F}$ и $\mathscr{L}$ - произвольные локально выпуклые топологические пространства. Через $\mathbb{L}(\mathscr{F} \rightarrow \mathscr{L})$ обозначим пространство всех линейных непрерывных отображений из $\mathscr{F}$ в $\mathscr{L}$. В частности, $\mathscr{D}^{\prime}=\mathbb{L}(\mathscr{D} \rightarrow \mathbb{C})$, а $\mathscr{S}^{\prime}=\mathbb{L}(\mathscr{S} \rightarrow \mathbb{C})$. Если $\mathscr{L}$ - произвольное локально вьпуклое пространство, то пространство $\mathbb{L}(\mathscr{D} \rightarrow \mathscr{L})$ можно трактовать как пространство обобщенных функций над $\mathscr{D}$ со значениями в $\mathscr{L}$. Так же можно интерпретировать пространства $\mathbb{L}\left(E^{\uparrow} \rightarrow \mathscr{L}\right), \mathbb{L}(\mathscr{S} \rightarrow \mathscr{L})$ и т.д.

Пусть $\mathscr{L}$ - некоторое локально выпуклое топологическое пространство и $f \in$ $\mathbb{L}(\mathscr{D} \rightarrow \mathscr{L})$. Для $\varphi \in \mathscr{D}$ положим

$$
L_{f}^{\varphi}(x, y)=\left(f(\xi), \frac{1}{y} \varphi\left(\frac{x-\xi}{y}\right)\right), \quad(x, y) \in \mathbb{R}^{1} \times(0,1] .
$$

Эта формула также имеет смысл, если $f \in \mathbb{L}\left(E^{\uparrow} \rightarrow \mathscr{L}\right), \mathbb{L}(\mathscr{S} \rightarrow \mathscr{L}), \mathbb{L}\left(E^{\downarrow} \rightarrow \mathscr{L}\right)$, а $\varphi \in E^{\uparrow}, \mathscr{S}, E^{\downarrow}$ соответственно. Очевидно, что $L_{f}^{\varphi}(x, y)$ - непрерывная функция в 
$\mathbb{R}^{1} \times(0,1]$ со значениями в $\mathscr{L}$. В частном случае, когда $\mathscr{L}=\mathbb{B}$, где $\mathbb{B}$ - банахово пространство, следуюшие оценки являются прямым следствием конечности порядка функционала на пространствах $\mathscr{D}, E^{\uparrow}, E^{\downarrow}, \mathscr{S}$ и называются общими оценками класса:

1) пусть $f(\xi) \in \mathbb{L}(\mathscr{D} \rightarrow \mathbb{B})$; тогда для любых $a>0$ и $a_{1}>0$ сушествуют $C, N$ и $b$ такие, что

$$
\left\|L_{f}^{\varphi}(x, y)\right\|_{\mathbb{B}} \leqslant \frac{C}{y^{b}} \mathscr{P}_{N}[\varphi], \quad 0<y \leqslant 1, \quad|x|<a_{1}, \quad \forall \varphi \in \mathscr{D}_{a}
$$

2) пусть $f(\xi) \in \mathbb{L}\left(E^{\uparrow} \rightarrow \mathbb{B}\right) ;$ тогда для любых $\varepsilon>0$ и $\varepsilon_{1}>0$ сушествуют $C, N$ и $b$ такие, что

$$
\left\|L_{f}^{\varphi}(x, y)\right\|_{\mathbb{B}} \leqslant \frac{C}{y^{b}} e^{\varepsilon_{1}|x|} P_{\varepsilon, N}[\varphi], \quad 0<y \leqslant 1, \quad x \in \mathbb{R}, \quad \forall \varphi \in E_{\varepsilon}
$$

3) пусть $f \in \mathbb{L}\left(E^{\downarrow} \rightarrow \mathscr{L}\right)$; тогда сушествуют $C, N, a$ и $b$ такие, что

$$
\left\|L_{f}^{\varphi}(x, y)\right\|_{\mathbb{B}} \leqslant \frac{C}{y^{b}} e^{a|x|} P_{a, N}[\varphi], \quad 0<y \leqslant 1, \quad x \in \mathbb{R}, \quad \forall \varphi \in E^{\downarrow}
$$

4) пусть $f(\xi) \in \mathbb{L}(\mathscr{S} \rightarrow \mathbb{B})$; тогда существуют $C, N, a$ и $b$ такие, что

$$
\left\|L_{f}^{\varphi}(x, y)\right\|_{\mathbb{B}} \leqslant \frac{C}{y^{b}}(1+|x|)^{a} \mathscr{P}_{N}[\varphi], \quad 0<y \leqslant 1, \quad x \in \mathbb{R}, \quad \forall \varphi \in \mathscr{S} .
$$

Для доказательства (1.13) см. лемму П.5, изложенную в приложении. Оценки (1.14)-(1.16) проверяются аналогично.

Положительная и непрерывная при $k \geqslant k_{0}$ функция $\rho(k)$ называется автомодельной (правильно меняющейся), если существует предел

$$
\lim _{k \rightarrow+\infty} \frac{\rho(k t)}{\rho(k)}=\psi(t)
$$

причем сходимость равномерная по $t$ на каждом компакте полуоси $(0, \infty)$. Легко видеть, что $\psi(t)=t^{\alpha}$ при некотором $\alpha$. В этом случае функцию $\rho(k)$ называем автомодельной порядка $\alpha$.

Отметим следуюшее свойство этих функций: для любого $\varepsilon>0$ существуют $C_{\varepsilon}$ и $c_{\varepsilon}$ такие, что

$$
c_{\varepsilon} \varkappa^{\alpha \mp \varepsilon} \leqslant \frac{\rho(\varkappa k)}{\rho(k)} \leqslant C_{\varepsilon} \varkappa^{\alpha \pm \varepsilon}, \quad k>k_{0}, \quad k \varkappa>k_{0}
$$

Здесь верхний знак берется при $\varkappa \geqslant 1$, а нижний - при $\varkappa<1$ (см. [3], [4]).

Автомодельную функцию можно доопределить на отрезке $\left[0, k_{0}\right]$ так, чтобы она стала непрерывной и положительной на всей замкнутой положительной полуоси. 
Тогда ясно, что в (1.17) можно считать $k_{0}=1$, кроме того, для любого $\varepsilon>0$ существует $C_{\varepsilon}$ такое, что при $k>1$

$$
\frac{\rho(\varkappa k)}{\rho(k)} \leqslant C_{\varepsilon} \gamma(\varkappa), \quad \gamma(\varkappa)= \begin{cases}\varkappa^{\alpha+\varepsilon}, & \varkappa \geqslant 1, \\ \varkappa^{-|\alpha|-\varepsilon}, & \varkappa<1 .\end{cases}
$$

Напомним некоторые сведения из теории интегралов Бохнера. Пусть $\mathbb{B}-$ банахово пространство и $\mu(x)$ - мера на открытом множестве $\Omega \subset \mathbb{R}^{n}$, абсолютно непрерывная относительно меры Лебега. Функция $h(x)$ называется простой, если

$$
h(x)=\sum_{i=1}^{N} b_{i} \chi_{\Omega_{i}}(x), \quad \bigcup_{i} \Omega_{i}=\Omega, \quad \Omega_{j} \cap \Omega_{i}=\varnothing,
$$

где $\Omega_{i}$ - измеримые множества, а $\chi_{\Omega_{i}}(x)$ - их характеристические функции. Функция $f(x)$ со значениями в $\mathbb{B}$ называется (сильно) измеримой, если она есть предел простых функций для почти всех $x \in \Omega$. Множество измеримых функций обозначим через $\mathscr{M}(\Omega \rightarrow \mathbb{B})$. Если $f \in \mathscr{M}(\Omega \rightarrow \mathbb{B})$, то $\|f(x)\|_{\mathbb{B}}$ - обычная измеримая функция. Обозначим

$$
L^{1}(\Omega \rightarrow \mathbb{B}, \mu)=\left\{f(x) \in \mathscr{M}(\Omega \rightarrow \mathbb{B}): \int_{\Omega}\|f(x)\|_{\mathbb{B}} d \mu(x)<\infty\right\} .
$$

Это - банахово пространство, и множество простых функций плотно в нем. Для простой функции $h(x)$ интеграл Бохнера определяется по формуле

$$
\int_{\Omega} h(x) d \mu(x)=\sum_{i=1}^{N} b_{i} \mu\left(\Omega_{i}\right) .
$$

Если последовательность простых функций $h_{n}(x) \rightarrow f(x)$ при $n \rightarrow \infty$ в $L^{1}(\Omega \rightarrow$ $\mathbb{B}, \mu)$, то последовательность $\int_{\Omega} h_{n}(x) d \mu(x)$ фундаментальна в $\mathbb{B}$ и ее предельный элемент, обозначаемый как $\int_{\Omega} f(x) d \mu(x)$, называют интегралом Бохнера. Имеют место следуюшие свойства интеграла Бохнера:

$$
\left\|\int_{\Omega} f(x) d \mu(x)\right\|_{\mathbb{B}} \leqslant \int_{\Omega}\|f(x)\|_{\mathbb{B}} d \mu(x) .
$$

Теорема ФУБини. Пусть $\Omega=\Omega_{1} \times \Omega_{2}$, где $\Omega_{1} \subset \mathbb{R}^{n}, \Omega_{2} \subset \mathbb{R}^{m}$, a $d \mu=$ $d \mu_{1}(x) \times d \mu_{2}(y)$, где $\mu_{i}-$ меры на $\Omega_{i}, \quad i=1,2$. Eсли $f(x, y) \in L^{1}(\Omega \rightarrow \mathbb{B})$, то для почти всех $x \in \Omega_{1}$ функция $f(x, y)$ интегрируема по Бохнеру на $\Omega_{2}$ по мере $\mu_{2}(y)$, функция $g(x)=\int_{\Omega_{2}} f(x, y) d \mu_{2}(y)$ измерима и интегрируема по Бохнеру на $\Omega_{1}$ по мере $\mu_{1}(x)$, причем

$$
\int_{\Omega} f(x, y) d \mu=\int_{\Omega_{1}} d \mu_{1}(x) \int_{\Omega_{2}} f(x, y) d \mu_{2}(y)
$$

(cM. $[10] u[11])$. 
Пусть $\mathbb{B}=A^{*}$, где $A$ - сепарабельное банахово пространство. Функция $f(x)$, $x \in \Omega$, принимающая значения в банаховом пространстве $\mathbb{B}$, называется $*$-слабо измеримой, если для любого $a \in A$ функция $\langle f(x), a\rangle$ измерима. Множество *-слабо измеримых функций обозначим * $\mathscr{M}(\Omega \rightarrow \mathbb{B})$. Если $f(x) \in{ }_{*} \mathscr{M}(\Omega \rightarrow \mathbb{B})$, то $\|f(x)\|_{\mathbb{B}}$ - обычная измеримая функция. Пусть

$$
{ }_{*} L^{1}(\Omega \rightarrow \mathbb{B}, \mu)=\left\{f(x) \in{ }_{*} \mathscr{M}(\Omega \rightarrow \mathbb{B}): \int_{\Omega}\|f(x)\|_{\mathbb{B}} d \mu(x)<\infty\right\} .
$$

Для $f(x) \in{ }_{*} L^{1}(\Omega \rightarrow \mathbb{B}, \mu)$ формула

$$
\langle I, a\rangle=\int_{\Omega}\langle f(x), a\rangle d \mu(x) \quad \forall a \in A
$$

определяет линейный непрерьвньй функционал на $A$, т.е. элемент из $\mathbb{B}$. Этот элемент называется $*$-слабым интегралом Бохнера и обозначается $\int_{\Omega} f(x) d \mu(x)$. Для $*$-слабого интеграла Бохнера справедлива теорема Фубини. Ясно, что интеграл Бохнера (если он существует) будет и $*$-слабым интегралом Бохнера.

Замыкание $\mathscr{D}$ по норме банахова пространства $V$ будем обозначать

$$
\left.(V)_{0} \quad \text { (иногда еще } \overline{\mathscr{D}}^{V}\right) .
$$

\section{§ 2. Автомодельные функционалы}

Пространство измеримых неотрицательных функций на положительной полуоси обозначим $\mathscr{M}_{+}$.

Пусть $\mathbb{J}(\varphi(k))$ - нетривиальный функционал на $\mathscr{M}_{+}$с носителем на $[1,+\infty)$ со значениями в $[0,+\infty]$, принимающий конечные значения на финитных ограниченных функциях со свойствами:

1) обобщенное неравенство треугольника:

$$
\mathbb{J}\left(\int \varphi(k, y) d y\right) \leqslant \int \mathbb{J}(\varphi(k, y)) d y
$$

для любой неотрицательной измеримой по $(k, y)$ функции $\varphi(k, y)$;

2) однородность: $\mathbb{J}(\lambda \varphi(k))=\lambda \mathbb{J}(\varphi(k)), \lambda \geqslant 0$;

$3)$ монотонность: если $\varphi_{1}(k) \leqslant \varphi_{2}(k)$ п.в., то $\mathbb{J}\left(\varphi_{1}(k)\right) \leqslant \mathbb{J}\left(\varphi_{2}(k)\right)$;

$4)$ если $\varphi_{n}(k) \nearrow \varphi(k)$ п.в., то $\mathbb{J}\left(\varphi_{n}(k)\right) \longrightarrow \mathbb{J}(\varphi(k)), n \rightarrow \infty$;

$5)$ сушествует $\alpha$ такое, что для любого $\varepsilon>0$ существует постоянная $C_{\varepsilon}$ такая, что

$$
\mathbb{J}(\varphi(\varkappa k)) \leqslant \begin{cases}C_{\varepsilon} \varkappa^{\alpha+\varepsilon} \mathbb{J}(\varphi(k)), & \text { если } \varkappa \geqslant 1, \\ C_{\varepsilon} \varkappa^{\alpha-\varepsilon} \mathbb{J}(\varphi(k)), & \text { если } \varkappa<1,\end{cases}
$$

для любой функции $\varphi(k)$ с носителем в $[1,+\infty)$.

Функционалы, удовлетворяюшие аксиомам 1)-5), будем называть автомодельныц.ми функционалами порядка $\alpha$. 
Ясно, что из обобщенного неравенства треугольников (аксиома 1)) вытекает обычное неравенство

$\left.1^{\prime}\right) \mathbb{J}\left(\varphi_{1}(k)+\varphi_{2}(k)\right) \leqslant \mathbb{J}\left(\varphi_{1}(k)\right)+\mathbb{J}\left(\varphi_{2}(k)\right), \varphi_{1}, \varphi_{2} \in \mathscr{M}_{+}$.

Представляет интерес выяснить, при каких условиях из $1^{\prime}$ ) вытекает 1).

ОПРЕДЕЛЕНИЕ 2.1. Будем говорить, что функционал $\mathbb{J}(\varphi)$, для которого выполнены условия $\left.\left.\left.1^{\prime}\right), 2\right)-5\right)$, удовлетворяет теореме Лебега, если из того, что $\varphi_{n}(k) \underset{n \rightarrow \infty}{\longrightarrow} \varphi(k)$ п.в. и из сушествования функции $\varphi_{0}(k)$ такой, что $\varphi_{n}(k) \leqslant$ $\varphi_{0}(k), \mathbb{J}\left(\varphi_{0}(k)\right)<\infty$, следует, что

$$
\mathbb{J}\left(\varphi_{n}(k)\right) \underset{n \rightarrow \infty}{\longrightarrow} \mathbb{J}(\varphi(k)) .
$$

Нетрудно доказать, что справедлива следующая

ЛЕмма 2.1. Пусть функционал $\mathbb{J}(\varphi)$ со свойствами 1'), 2)-5) удовлетворяет теореме Лебега. Тогда $\mathbb{J}-$ автомодельный функционал.

Приведем примеры автомодельных функционалов порядка $\alpha=0$. Положим

$$
\mathbb{J}^{p}(\varphi(k))=\left(\int_{1}^{\infty} \varphi^{p}(k) \frac{d k}{k}\right)^{\frac{1}{p}}, \quad 1 \leqslant p<\infty, \quad \mathbb{J}^{\infty}(\varphi(k))=\underset{k>1}{\operatorname{ess} \sup } \varphi(k) .
$$

Функционалы $\rrbracket^{p}(\varphi)$ при $1 \leqslant p<\infty$ удовлетворяют теореме Лебега. Функционал $\mathbb{J}^{\infty}(\varphi)$ не удовлетворяет теореме Лебега, но, тем не менее, для него выполняется обобщенное неравенство треугольника.

Приведем некоторые свойства автомодельных функционалов. Непосредственно из аксиомы 5) следует, что для любого $\varepsilon>0$ существует $C_{\varepsilon}$ такое, что

$$
\frac{1}{C_{\varepsilon}} \varkappa^{\alpha \mp \varepsilon} \mathbb{J}(\varphi(k)) \leqslant \mathbb{J}(\varphi(\varkappa k)), \quad \operatorname{supp} \varphi \subset[\varkappa,+\infty) .
$$

Здесь берется знак - , если $\varkappa \geqslant 1$, и знак + , если $\varkappa<1$. В частности, если $\varkappa<1$ и $\operatorname{supp} \varphi \subset[1,+\infty)$, то для любого $\varepsilon>0$ сушествует $C_{\varepsilon}$ такое, что

$$
\frac{1}{C_{\varepsilon}} \varkappa^{\alpha+\varepsilon} \mathbb{J}(\varphi(k)) \leqslant \mathbb{J}(\varphi(\varkappa k)) \leqslant C_{\varepsilon} \varkappa^{\alpha-\varepsilon} \mathbb{J}(\varphi(k)) .
$$

Отсюда следует, что порядок автомодельности функционала $\alpha$ определяется однозначно.

Отметим, что если $\varkappa \geqslant 1$, то аксиома 5) выполняется для любой функции $\varphi(k) \in$ $\mathscr{M}_{+}$. Действительно, пользуясь тождеством $1=\chi_{[0,1]}(\varkappa k)+\chi_{[1, \infty)}(\varkappa k)$, имеем

$$
\begin{aligned}
\mathbb{J}(\varphi(\varkappa k)) & \leqslant \mathbb{J}\left(\chi_{[1, \infty)}(\varkappa k) \varphi(\varkappa k)\right)+\mathbb{J}\left(\chi_{[0,1]}(\varkappa k) \varphi(\varkappa k)\right) \\
& \leqslant C_{\varepsilon} \varkappa^{\alpha+\varepsilon} \mathbb{J}(\varphi(k)) .
\end{aligned}
$$

Здесь мы воспользовались тем, что $\chi_{[0,1]}(\varkappa k)=0$ при $\varkappa \geqslant 1, k>1, \operatorname{a~supp} \mathbb{J}(\varphi) \subset$ $[1, \infty)$.

Как следствие аксиом 3) и 4) справедлив следующий аналог теоремы Фату. 
Teopema 2.1. Пусть $\varphi_{n} \in \mathscr{M}_{+} u \varphi_{n}(k) \rightarrow \varphi(k), \quad n \rightarrow+\infty$, почти всюду; mогда $\underline{\lim }_{n \rightarrow \infty} \mathbb{J}\left(\varphi_{n}\right) \geqslant \mathbb{J}(\varphi)$.

ЛЕмма 2.2. Пусть $\rho(k)$ - автомодельная функиия порядка $\beta$, а $\mathbb{J}(\varphi(k))-$ автомодельный функционал порядка $\alpha$. Положим $\mathbb{J}_{1}(\varphi(k))=\mathbb{J}\left(\frac{1}{\rho(k)} \varphi(k)\right)$. Тогда $\mathbb{J}_{1}-$ автомодельный функиионал порядка $\alpha+\beta$.

Действительно, достаточно проверить аксиому 5). Пусть $\operatorname{supp} \varphi \subset[1,+\infty)$. Пользуясь свойствами правильно меняющихся функций (см. (1.17)), имеем

$$
\begin{aligned}
\mathbb{J}_{1}(\varphi(\varkappa k)) & =\mathbb{J}\left(\frac{1}{\rho(k)} \varphi(\varkappa k)\right)=\mathbb{J}\left(\frac{\rho(\varkappa k)}{\rho(k)} \frac{1}{\rho(\varkappa k)} \varphi(\varkappa k)\right) \\
& \leqslant \mathbb{J}\left(C_{\varepsilon}^{1} \varkappa^{\beta \pm \varepsilon} \frac{1}{\rho(\varkappa k)} \varphi(\varkappa k)\right)=C_{\varepsilon}^{1} \varkappa^{\beta \pm \varepsilon} \mathbb{J}\left(\frac{1}{\rho(\varkappa k)} \varphi(\varkappa k)\right) \\
& \leqslant C_{\varepsilon}^{1} \varkappa^{\beta \pm \varepsilon} C_{\varepsilon}^{2} \varkappa^{\alpha \pm \varepsilon} \mathbb{J}\left(\frac{\varphi(k)}{\rho(k)}\right) \leqslant C_{\varepsilon} \varkappa^{\beta+\alpha \pm 2 \varepsilon} \mathbb{J}_{1}(\varphi(k)) .
\end{aligned}
$$

Здесь берется знак + , если $\varkappa \geqslant 1$, и знак - , если $\varkappa<1, C_{\varepsilon}^{1}$ берется из (1.17), а $C_{\varepsilon}^{2}-$ из аксиомы 5$), C_{\varepsilon}-$ некоторая постоянная.

ЛЕмма 2.3. Пусть $\mathbb{I}(\varphi(k))$ - автомодельный функционал порядка $\alpha ;$ тогда для любого $\varepsilon>0$ существует постоянная $C_{\varepsilon}$ такая, что

$$
\mathbb{J}(\varphi(k)) \leqslant C_{\varepsilon} \operatorname{ess~sup}_{k>1} \frac{\varphi(k)}{k^{\alpha-\varepsilon}}, \quad \varphi \in \mathscr{M}_{+} .
$$

ДокАЗАТЕЛЬСТво. Предположим, что $\alpha>0$. Тогда покажем, что $\mathbb{J}(1)<\infty$. Действительно, для любого $\varkappa>1$ при $n>\log _{2}([\varkappa]+1)$ в силу аксиом 3$)$ и 5$)$ имеем

$$
\begin{aligned}
\mathbb{J}\left(\chi_{[1, \varkappa]}(k)\right) & \leqslant \mathbb{J}\left(\chi_{\left[1,2^{n}\right]}(k)\right)=\mathbb{J}\left(\sum_{j=0}^{n-1} \chi_{[1,2]}\left(\frac{k}{2^{j}}\right)\right) \\
& \leqslant \sum_{j=0}^{n-1} \mathbb{J}\left(\chi_{[1,2]}\left(\frac{k}{2^{j}}\right)\right) \leqslant C_{\varepsilon} \sum_{j=0}^{n-1}\left(\frac{1}{2^{j}}\right)^{\alpha-\varepsilon} \mathbb{J}\left(\chi_{[1,2]}(k)\right) .
\end{aligned}
$$

В последнем неравенстве мы считаем, что $\alpha-\varepsilon>0$. Переходя к пределу при $\varkappa \rightarrow+\infty$, получим

$$
\mathbb{J}(1) \leqslant C_{\varepsilon} \sum_{j=0}^{\infty}\left(\frac{1}{2^{\alpha-\varepsilon}}\right)^{j} \mathbb{J}\left(\chi_{[1,2]}(k)\right)<\infty .
$$

Пусть теперь $\alpha$ произвольно. Порядок функционала $\mathbb{J}_{1}(\varphi(k))=\mathbb{J}\left(k^{\alpha-\varepsilon} \varphi(k)\right)$ согласно лемме 2.1 будет равен $\varepsilon>0$, а потому

$$
\mathbb{J}\left(k^{\alpha-\varepsilon} \psi(k)\right)=\mathbb{J}_{1}(1 \cdot \psi(k)) \leqslant \mathbb{J}_{1}(1) \operatorname{ess~sup}_{k>1} \psi(k) \leqslant C_{\varepsilon} \underset{k>1}{\operatorname{esssup}} \psi(k) .
$$

Полагая в этом неравенстве $\varphi(k)=k^{\alpha-\varepsilon} \psi(k)$, получим $(2.5)$. 
ЛЕмма 2.4 (неравенства Харди). Пусть Ј( $($ ) - автомодельный функиионал порядка $\alpha$. Тогда существует постоянная $C$ такая, ито

$$
C \mathbb{J}(\varphi(k)) \geqslant \begin{cases}\mathbb{J}\left(\int_{k}^{+\infty} \varphi(\varkappa) \frac{d \varkappa}{\varkappa}\right) & \text { nрu } \alpha<0, \\ \mathbb{J}\left(\int_{1}^{k} \varphi(\varkappa) \frac{d \varkappa}{\varkappa}\right) & \text { nрu } \alpha>0\end{cases}
$$

для любой функиии $\varphi(k) \in \mathscr{M}_{+}$.

ДоКАЗАТЕЛЬСтво. Без ограничения общности можно считать, что $\operatorname{supp} \varphi \subset$ $[1,+\infty)$. Пусть $\alpha>0$. Из аксиомы 5) при $0<y<1$ для достаточно малого $\varepsilon$ имеем

$$
\mathbb{J}\left(\frac{1}{y} \varphi(k y)\right) \leqslant C_{\varepsilon} \frac{1}{y} y^{\alpha-\varepsilon} \mathbb{J}(\varphi(k)) .
$$

Отсюда согласно обобщенной аксиоме треугольника получаем

$$
\begin{aligned}
\mathbb{J}\left(\int_{1}^{k} \varphi(\varkappa) \frac{d \varkappa}{\varkappa}\right) & =\mathbb{J}\left(\int_{0}^{k} \varphi(\varkappa) \frac{d \varkappa}{\varkappa}\right)=\mathbb{J}\left(\int_{0}^{1} \varphi(k y) \frac{d y}{y}\right) \\
& \leqslant \int_{0}^{1} \mathbb{J}\left(\frac{1}{y} \varphi(k y)\right) d y \leqslant C_{\varepsilon} \mathbb{J}(\varphi(k)) \int_{0}^{1} y^{\alpha-\varepsilon} \frac{d y}{y} \leqslant C \mathbb{J}(\varphi(k)) .
\end{aligned}
$$

Пусть теперь $\alpha<0$. Из аксиомы 5) при $1<y<+\infty$ для достаточно малого $\varepsilon$ имеем

$$
\mathbb{J}\left(\frac{1}{y} \varphi(k y)\right) \leqslant C_{\varepsilon} \frac{1}{y} y^{\alpha+\varepsilon} \mathbb{J}(\varphi(k)) .
$$

Отсюда, аналогично предыдущему, получаем

$$
\mathbb{J}\left(\int_{k}^{\infty} \varphi(\varkappa) \frac{d \varkappa}{\varkappa}\right)=\mathbb{J}\left(\int_{1}^{\infty} \varphi(k y) \frac{d y}{y}\right) \leqslant C_{\varepsilon} \mathbb{J}(\varphi(k)) \int_{1}^{\infty} y^{\alpha+\varepsilon} \frac{d y}{y} \leqslant C \mathbb{J}(\varphi(k)) .
$$

Лемма доказана.

ЛЕмма 2.5. Пусть $\mathbb{J}(\varphi)$ - автомодельньй функционал порядка $\alpha$. Тогда для любого $\varepsilon>0$ существует постоянная $C$ такая, что

$$
\int_{1}^{\infty} \frac{\varphi(k)}{k^{\alpha+\varepsilon}} \frac{d k}{k} \leqslant C \mathbb{J}(\varphi(k))
$$

для любой функции $\varphi(k) \in \mathscr{M}_{+}$.

ДоКАЗАТЕЛЬСТвО. Пусть $\alpha>0$. Фиксируя $\varepsilon>0$, заметим, что при $\varkappa>1$

$$
\chi_{[\varkappa, \infty]}(k) \int_{1}^{\infty} \chi_{[1, \varkappa]}(\xi) \varphi(\xi) \frac{d \xi}{\xi} \leqslant \int_{1}^{k} \varphi(\xi) \frac{d \xi}{\xi},
$$

а потому

$$
\int_{1}^{\infty} \chi_{[1, \varkappa]}(\xi) \varphi(\xi) \frac{d \xi}{\xi} \mathbb{J}\left(\chi_{[\varkappa, \infty]}(k)\right) \leqslant \mathbb{J}\left(\int_{1}^{k} \varphi(\xi) \frac{d \xi}{\xi}\right) \leqslant C \mathbb{J}(\varphi(k)),
$$


здесь и далее $C$ обозначает некоторую постоянную, которая может меняться от формулы к формуле. В последнем неравенстве мы воспользовались леммой 2.4. Согласно формуле $(2.3)$, меняя $\varepsilon$ на $\frac{\varepsilon}{2}$ и $\varkappa$ на $\frac{1}{\varkappa}$, имеем

$$
\mathbb{J}\left(\chi_{[\varkappa, \infty]}(k)\right) \geqslant \mathbb{J}\left(\chi_{[1,2]}\left(\frac{k}{\varkappa}\right)\right) \geqslant C\left(\frac{1}{\varkappa}\right)^{\alpha+\frac{\varepsilon}{2}} \mathbb{J}\left(\chi_{[1,2]}(k)\right) .
$$

Теперь из (2.8) получаем оценку

$$
\int_{1}^{\infty} \chi_{[1, \varkappa]}(\xi) \varphi(\xi) \frac{d \xi}{\xi} \leqslant C \varkappa^{\alpha+\frac{\varepsilon}{2}} \mathbb{J}(\varphi(k)) .
$$

Пользуясь ею, имеем

$$
\begin{aligned}
\int_{1}^{\infty} \chi_{\left[2^{j}, 2^{j+1}\right]}(\xi) \frac{\varphi(\xi)}{\xi^{\alpha+\varepsilon}} \frac{d \xi}{\xi} & \leqslant \frac{1}{2^{j(\alpha+\varepsilon)}} \int_{1}^{\infty} \chi_{\left[1,2^{j+1}\right]}(\xi) \varphi(\xi) \frac{d \xi}{\xi} \\
& \leqslant C_{1} \frac{1}{2^{j(\alpha+\varepsilon)}} 2^{(j+1)\left(\alpha+\frac{\varepsilon}{2}\right)} \mathbb{J}(\varphi(k))=\frac{2^{\alpha+\frac{\varepsilon}{2}} C_{2}}{\left(2^{\frac{\varepsilon}{2}}\right)^{j}} \mathbb{J}(\varphi(k)) \\
& \leqslant \frac{C_{3}}{\left(2^{\frac{\varepsilon}{2}}\right)^{j}} \mathbb{J}(\varphi(k)),
\end{aligned}
$$

где $C_{1}, C_{2}, C_{3}$ - некоторые постоянные. Далее получаем

$$
\begin{aligned}
\int_{1}^{\infty} \frac{\varphi(k)}{k^{\alpha+\varepsilon}} \frac{d k}{k} & =\int_{1}^{\infty} \sum_{j=0}^{\infty} \chi_{\left[2^{j}, 2^{j+1}\right]}(\xi) \frac{\varphi(\xi)}{\xi^{\alpha+\varepsilon}} \frac{d \xi}{\xi} \\
& =\sum_{j=0}^{\infty} \int_{1}^{\infty} \chi_{\left[2^{j}, 2^{j+1}\right]}(\xi) \frac{\varphi(\xi)}{\xi^{\alpha+\varepsilon}} \frac{d \xi}{\xi} \leqslant \sum_{j=0}^{\infty} \frac{C}{\left(2^{\frac{\varepsilon}{2}}\right)^{j}} \mathbb{J}(\varphi(k)) \leqslant C \mathbb{J}(\varphi(k))
\end{aligned}
$$

Пусть теперь $\alpha \leqslant 0$. Возьмем $\beta>0$ и положим $\mathbb{J}_{1}(\varphi)=\mathbb{J}\left(\frac{1}{k^{\beta-\alpha}} \varphi(k)\right)$. Из леммы 2.2 вытекает, что $\mathbb{J}_{1}(\varphi)$ - автомодельный функционал порядка $\beta>0$. А потому, пользуясь уже доказанньм, для любой неотрищательной измеримой функции $\psi(k)$ имеем

$$
\int_{1}^{\infty} \frac{\psi(k)}{k^{\beta+\varepsilon}} \frac{d k}{k} \leqslant C \mathbb{J}_{1}(\psi(k))=C \mathbb{J}\left(\frac{1}{k^{\beta-\alpha}} \psi(k)\right) .
$$

Полагая здесь $\psi(k)=\frac{1}{k^{\alpha-\beta}} \varphi(k)$, заканчиваем доказательство леммы.

Суммируем полученные результаты в виде следующего утверждения.

УТВЕРЖДЕНИЕ 2.1. Пусть $\mathbb{J}(\varphi)$ - автомодельный функиионал порядка $\alpha$. Тогда для любого є> 0 существуют постоянные $C$ и с такие, ито

$$
c \mathbb{J}^{1}\left(\frac{\varphi(k)}{k^{\alpha+\varepsilon}}\right) \leqslant \mathbb{J}(\varphi(k)) \leqslant C \mathbb{J}^{\infty}\left(\frac{\varphi(k)}{k^{\alpha-\varepsilon}}\right) \quad \forall \varphi \in \mathscr{M}_{+} .
$$

Наряду с автомодельным функционалом $\mathbb{J}(\cdot)$ удобно рассматривать соответствующий ему функционал $\hat{\mathbb{J}}(\cdot)$. 
ОПРЕДЕЛЕНИЕ 2.2. Пусть $\mathbb{J}(\varphi(k))$ - автомодельньй функционал. Тогда для любой $\psi(y) \in \mathscr{M}_{+}$положим

$$
\hat{\mathbb{J}}(\psi(y))=\mathbb{J}\left(\psi\left(\frac{1}{k}\right)\right) .
$$

Очевидно, что $\hat{\mathbb{J}}(\cdot)$ сосредоточен на полуинтервале $(0,1]$, т.е. $\hat{\mathbb{J}}(\psi(y))=0$, если $\operatorname{supp} \psi(y) \subset[1,+\infty)$.

ЗАмечАниЕ 2.1. Пусть $\mathbb{B}$ - банахово пространство, $\mathbb{J}$ - автомодельньй функционал. Пусть

$$
L^{\mathbb{J}}([1,+\infty) \rightarrow \mathbb{B})=\left\{f(k) \in \mathscr{M}([1,+\infty) \rightarrow \mathbb{B}): \mathbb{J}\left(\|f(k)\|_{\mathbb{B}}\right)<\infty\right\} .
$$

Пользуясь оценками $(2.9)$, нетрудно показать, что $L^{\mathbb{J}}([1,+\infty) \rightarrow \mathbb{B})-$ банахово пространство.

Если $f_{n}(\eta, k) \underset{n \rightarrow+\infty}{\longrightarrow} f_{\infty}(\eta, k)$ в $L^{\mathbb{J}}([1,+\infty) \rightarrow \mathbb{B})$, то можно выделить подпоследовательность $f_{n_{m}}(\eta, k) \underset{m \rightarrow+\infty}{\longrightarrow} f_{\infty}(\eta, k)$ в $\mathbb{B}$ при почти всех $k \in[1,+\infty)$.

Ясно, что $L^{\mathbb{J}}([1,+\infty) \rightarrow \mathbb{B})$ изоморфно пространству $L^{\hat{\mathbb{J}}}((0,1] \rightarrow \mathbb{B})$, состоящему из функций $f(\eta, y)$, измеримых по $y \in(0,1)$, со значениями в банаховом пространстве $\mathbb{B}$, и таких, что $\hat{J}\left(\|f(\cdot, y)\|_{\mathbb{B}}\right)<\infty$.

ЗАмЕчАниЕ 2.2. Если порядок автомодельного функционала $\mathbb{ل}(\cdot)$ больше нуля, то из $(2.9)$ следует, что $\mathbb{J}\left(\frac{1}{k^{j}}\right)<\infty$ при $j \geqslant 0$.

Для обобшенных функций со значениями в банаховом пространстве $\mathbb{B}$ справедливы общие оченки класса относительно автомодельного функционала $\mathbb{J}$. А именно, пусть $\mathbb{J}(\cdot)$ - автомодельный функционал.

1) Если $f \in \mathbb{L}(\mathscr{D} \rightarrow \mathbb{B})$, то для любых $a>0$ и $a_{1}>0$ существуют $C, N$ и $b$ такие, что

$$
\hat{\mathbb{J}}\left(y^{b}\left\|L_{f}^{\varphi}(x, y)\right\|_{\mathbb{B}}\right)=\mathbb{J}\left(\frac{1}{k^{b}}\left\|L_{f}^{\varphi}\left(x, \frac{1}{k}\right)\right\|_{\mathbb{B}}\right) \leqslant C \mathscr{P}_{N}[\varphi],|x|<a_{1}, \forall \varphi \in \mathscr{D}_{a} .
$$

2) Если $f \in \mathbb{L}\left(E^{\uparrow} \rightarrow \mathbb{B}\right)$, то для любых $\varepsilon>0$ и $\varepsilon_{1}>0$ сушествуют $C, N$ и $b$ такие, что

$$
\hat{\mathbb{J}}\left(y^{b}\left\|L_{f}^{\varphi}(x, y)\right\|_{\mathbb{B}}\right)=\mathbb{J}\left(\frac{1}{k^{b}}\left\|L_{f}^{\varphi}\left(x, \frac{1}{k}\right)\right\|_{\mathbb{B}}\right) \leqslant C e^{\varepsilon_{1}|x|} P_{\varepsilon, N}[\varphi], \quad x \in \mathbb{R}, \quad \forall \varphi \in E_{\varepsilon} .
$$

3) Если $f \in \mathbb{L}\left(E^{\downarrow} \rightarrow \mathbb{B}\right)$, то сушествуют $a, b, C$ и $N$ такие, что

$$
\hat{\mathbb{J}}\left(y^{b}\left\|L_{f}^{\varphi}(x, y)\right\|_{\mathbb{B}}\right)=\mathbb{J}\left(\frac{1}{k^{b}}\left\|L_{f}^{\varphi}\left(x, \frac{1}{k}\right)\right\|_{\mathbb{B}}\right) \leqslant C e^{a|x|} P_{a, N}[\varphi], \quad x \in \mathbb{R}, \quad \forall \varphi \in E^{\downarrow} .
$$

4) Если $f \in \mathbb{L}(\mathscr{S} \rightarrow \mathbb{B})$, то существуют $a, b, C$ и $N$ такие, что

$$
\hat{\mathbb{J}}\left(y^{b}\left\|L_{f}^{\varphi}(x, y)\right\|_{\mathbb{B}}\right)=\mathbb{J}\left(\frac{1}{k^{b}}\left\|L_{f}^{\varphi}\left(x, \frac{1}{k}\right)\right\|_{\mathbb{B}}\right) \leqslant C(1+|x|)^{a} \mathscr{P}_{N}[\varphi], \quad x \in \mathbb{R}, \quad \forall \varphi \in \mathscr{S} .
$$

Доказательство этих оценок следует из неравенств (1.13)-(1.16) и утверждения 2.1 (см., например, лемму П.5). 


\section{§ 3. Основные теоремы}

В этом параграфе докажем тауберовы теоремы, касаюшиеся обобшенных функций над $\mathscr{D}$ со значениями в банаховом пространстве $\mathbb{B}$, т.е. для $f \in \mathbb{L}(\mathscr{D} \rightarrow \mathbb{B})$. Аналогичные теоремы справедливы и для пространств $\mathbb{L}\left(E^{\uparrow} \rightarrow \mathbb{B}\right), \mathbb{L}\left(E^{\downarrow} \rightarrow \mathbb{B}\right)$ и $\mathbb{L}(\mathscr{S} \rightarrow \mathbb{B})$. Далее будут приведены их формулировки.

Пусть $\mathscr{L}$ - некоторое локально выпуклое топологическое пространство. Напомним, что для $\omega \in \mathscr{D}$

$$
L_{f}^{\omega}(x, y)=\left(f(\xi), \frac{1}{y} \omega\left(\frac{x-\xi}{y}\right)\right), \quad(x, y) \in \mathbb{R}^{1} \times(0,1] .
$$

Очевидно, что $L_{f}^{\varphi}(x, y)$ - непрерьвная функция в $\mathbb{R}^{1} \times(0,1]$ со значениями в $\mathscr{L}$.

ТЕОремА 3.1. Пусть $\mathbb{J}$ - автомодельный функционал, $\mathbb{B}$ - банахово и $\mathscr{L}$ локально выпуклое пространства, причем $\mathbb{B} \subset \mathscr{L}$ линейно и непрерывно. Пусть $f \in \mathbb{L}(\mathscr{D} \rightarrow \mathscr{L})$ и для некоторой $\omega(\xi) \in \stackrel{n}{\mathscr{D} \backslash} \stackrel{n}{\mathscr{D}}^{+1}$ выполнены условия: $L_{f}^{\omega}(x, y) \in \mathbb{B}$ при п.в. $(x, y) \in \mathbb{R}^{1} \times(0,1]$ и является измеримой функиией со значениями в $\mathbb{B}$, причем для любого $d>0$ найдется $b$ такое, что

$$
\underset{|x|<d}{\operatorname{ess} \sup } \hat{\mathbb{J}}\left(y^{b}\left\|L_{f}^{\omega}(x, y)\right\|_{\mathbb{B}}\right)<\infty
$$

Тогда

$$
(f(\xi), \varphi(\xi)) \in \mathbb{B} \quad \forall \varphi \in \stackrel{n}{\mathscr{D}} .
$$

Более того, для любого $а>0$ существует $a_{1}$ такое, что для любого $b$ найдутся $C$ и $N$ такие, что

$$
\|(f(\xi), \varphi(\xi))\|_{\mathbb{B}} \leqslant C \underset{|x|<a_{1}}{\operatorname{ess} \sup } \hat{\mathbb{J}}\left(y^{b}\left\|L_{f}^{\omega}(x, y)\right\|_{\mathbb{B}}\right) \mathscr{P}_{N}[\varphi] \quad \forall \varphi \in \stackrel{n}{\mathscr{D}_{a}}
$$

әде $a_{1}, C$ и $N$ не зависят от $\varphi, \mathbb{B} u f$.

Если $n \geqslant 0$ и для некоторой функиии $\omega_{0} \in \mathscr{D} \backslash \stackrel{0}{\mathscr{D}}$ функция $L_{f}^{\omega_{0}}(x, 1)$ измерима по $x$ как функиия со значениями в $\mathbb{B} u$

$$
\underset{|x|<d}{\operatorname{ess} \sup }\left\|L_{f}^{\omega_{0}}(x, 1)\right\|_{\mathbb{B}}<\infty \quad \forall d>0,
$$

mo $f \in \mathbb{L}(\mathscr{D} \rightarrow \mathbb{B})$.

Более того, для любого $a>0$ существует $a_{1}$ такое, что для любого $b$ найдутся $C$ и $N$ такие, что

$$
\begin{aligned}
& \|(f(\xi), \varphi(\xi))\|_{\mathbb{B}} \leqslant \\
& \leqslant C\left[\underset{|x|<a_{1}}{\operatorname{ess} \sup _{\mathbb{J}}} \hat{\mathbb{J}}\left(y^{b}\left\|L_{f}^{\omega}(x, y)\right\|_{\mathbb{B}}\right)+\underset{|x|<a_{1}}{\operatorname{ess} \sup _{f}}\left\|L_{f}^{\omega_{0}}(x, 1)\right\|_{\mathbb{B}}\right] \mathscr{P}_{N}[\varphi] \quad \forall \varphi \in \mathscr{D}_{a},(3
\end{aligned}
$$

әде $a_{1}, C$ и $N$ не зависят от $\varphi, \mathbb{B} u f$. 
ДокАЗАТЕЛЬСТво. Пусть $\alpha$ - порядок автомодельности функционала ل. Фиксируем любое $d>0$. Делая замену переменных $y=\frac{1}{k}$, учитывая (2.9) и (3.2), получаем

$$
\begin{aligned}
\int_{0}^{1} y^{\alpha+b+\varepsilon}\left\|L_{f}^{\omega}(x, y)\right\|_{\mathbb{B}} \frac{d y}{y} & =\int_{1}^{\infty} \frac{1}{k^{\alpha+b+\varepsilon}}\left\|L_{f}^{\omega}\left(x, \frac{1}{k}\right)\right\|_{\mathbb{B}} \frac{d k}{k} \\
& \leqslant C \mathbb{J}\left(\frac{1}{k^{b}}\left\|L_{f}^{\omega}\left(x, \frac{1}{k}\right)\right\|_{\mathbb{B}}\right)=C \hat{\mathbb{J}}\left(y^{b}\left\|L_{f}^{\omega}(x, y)\right\|_{\mathbb{B}}\right) .
\end{aligned}
$$

Отсюда, полагая $s>\alpha+b+1$ и $0<\beta \leqslant 1$, для п.в. $|x|<d$ имеем

$$
\left\|\int_{0}^{\beta} y^{s} L_{f}^{\omega}(x, y) d y\right\|_{\mathbb{B}} \leqslant \int_{0}^{\beta} y^{s}\left\|L_{f}^{\omega}(x, y)\right\|_{\mathbb{B}} d y<\underset{|x|<d}{\operatorname{ess} \sup } \hat{\mathbb{J}}\left(y^{s}\left\|L_{f}^{\omega}(x, y)\right\|_{\mathbb{B}}\right) .
$$

Здесь первый интеграл есть интеграл Бохнера, и так как

$$
\int_{-d}^{d} d x \int_{0}^{\beta} y^{s}\left\|L_{f}^{\omega}(x, y)\right\|_{\mathbb{B}} d y<\infty
$$

то $\int_{0}^{\beta} y^{s} L_{f}^{\omega}(x, y) d y \in L^{1}((-d, d) \rightarrow \mathbb{B})$. Поэтому для любой функции $\chi(x) \in \mathscr{D}_{d}$ интеграл Бохнера $\int \chi(x) \int_{0}^{\beta} y^{s} L_{f}^{\omega}(x, y) d y d x$ принадлежит $\mathbb{B}$ и справедлива оценка

$$
\left\|\int \chi(x) \int_{0}^{\beta} y^{s} L_{f}^{\omega}(x, y) d y d x\right\|_{\mathbb{B}} \leqslant C \underset{|x|<d}{\operatorname{ess} \sup } \hat{\mathbb{J}}\left(y^{b}\left\|L_{f}^{\omega}(x, y)\right\|_{\mathbb{B}}\right) \int|\chi(x)| d x .
$$

Пользуясь теоремой Фубини для интегралов Бохнера (для функций со значениями в банаховом пространстве), имеем

$$
\begin{aligned}
\int \chi(x) \int_{0}^{\beta} y^{s} L_{f}^{\omega}(x, y) d y d x & =\int_{0}^{\beta} y^{s} \int \chi(x) L_{f}^{\omega}(x, y) d x d y \\
& =\int_{0}^{\beta} y^{s} d y \int \chi(x)\left(f(\xi), \frac{1}{y} \omega\left(\frac{x-\xi}{y}\right)\right) d x \\
& =\int_{0}^{\beta} y^{s} d y \int \chi(x)\left(\tilde{f}(t), e^{i x t} \widetilde{\omega}(y t)\right) d x
\end{aligned}
$$

В частности, при почти всех $y \in(0,1]$

$$
\int \chi(x)\left(\tilde{f}(t), e^{i x t} \widetilde{\omega}(y t)\right) d x \in \mathbb{B} \quad \forall \chi(x) \in \mathscr{D}_{d}
$$

Здесь мы пользовались формулой (3.1) и в последнем равенстве (3.7) перешли к преобразованию Фурье, учитывая формулу

$$
\left(f(\xi), \frac{1}{y} \omega\left(\frac{x-\xi}{y}\right)\right)=\left(F[f(\xi)](t), F\left[\frac{1}{y} \omega\left(\frac{x-\xi}{y}\right)\right](t)\right)=\left(\tilde{f}(t), e^{i x t} \widetilde{\omega}(y t)\right) .
$$


Волной обозначаем преобразование Фурье, так что $\tilde{f}(t) \in Z^{\prime}$ и $\widetilde{\omega}(t) \in Z_{n}$, где $\operatorname{supp} \omega(\xi) \subset[-\gamma, \gamma]$.

Теперь покажем, что почти всюду по $y$ (при тех $y \in(0,1]$, при которых $(3.8)$ имеет место) выполняется равенство

$$
\int \chi(x)\left(\tilde{f}(t), e^{i x t} \widetilde{\omega}(y t)\right) d x=\left(\tilde{f}(t), \int \chi(x) e^{i x t} \widetilde{\omega}(y t) d x\right)
$$

для элементов пространства $\mathscr{L}$.

Действительно, достаточно показать, что для любой функции $\psi \in \mathscr{L}^{*}$

$$
\left\langle\int \chi(x)\left(\tilde{f}(t), e^{i x t} \widetilde{\omega}(y t)\right) d x, \psi\right\rangle=\left\langle\left(\tilde{f}(t), \int \chi(x) e^{i x t} \widetilde{\omega}(y t) d x\right), \psi\right\rangle .
$$

Так как слева стоит интеграл Бохнера в $\mathbb{B}$, а $\psi \in \mathscr{L}^{*} \subset \mathbb{B}^{*}($ напомним, что $\mathbb{B} \subset \mathscr{L}$ ), то

$$
\left\langle\int \chi(x)\left(\tilde{f}(t), e^{i x t} \widetilde{\omega}(y t)\right) d x, \psi\right\rangle=\int \chi(x)\left\langle\left(\tilde{f}(t), e^{i x t} \widetilde{\omega}(y t)\right), \psi\right\rangle d x .
$$

Рассмотрим выражение, стоящее справа в (3.9). Так как для интегральных сумм выполенно соотношение

$$
\sum_{j} \chi\left(x_{j}\right) e^{i x_{j} t} \widetilde{\omega}(y t) \Delta_{j} \underset{\Delta \rightarrow 0}{\longrightarrow} \chi \chi(x) e^{i x t} \widetilde{\omega}(y t) d x \quad \text { в } Z
$$

то имеем

$$
\left(\tilde{f}(t), \sum_{j} \chi\left(x_{j}\right) e^{i x_{j} t} \widetilde{\omega}(y t) \Delta_{j}\right) \underset{\Delta \rightarrow 0}{\longrightarrow}\left(\tilde{f}(t), \int \chi(x) e^{i x t} \widetilde{\omega}(y t) d x\right) \text { в } \mathscr{L} .
$$

Поэтому

$$
\begin{aligned}
\left\langle\left(\tilde{f}(t), \int \chi(x) e^{i x t} \widetilde{\omega}(y t) d x\right), \psi\right\rangle & =\left\langle\lim _{\Delta \rightarrow 0}\left(\tilde{f}(t), \sum_{j} \chi\left(x_{j}\right) e^{i x_{j} t} \widetilde{\omega}(y t) \Delta_{j}\right), \psi\right\rangle \\
& =\lim _{\Delta \rightarrow 0}\left\langle\left(\tilde{f}(t), \sum_{j} \chi\left(x_{j}\right) e^{i x_{j} t} \widetilde{\omega}(y t) \Delta_{j}\right), \psi\right\rangle \\
& =\lim _{\Delta \rightarrow 0} \sum_{j} \chi\left(x_{j}\right)\left\langle\left(\tilde{f}(t), e^{i x_{j} t} \widetilde{\omega}(y t)\right), \psi\right\rangle \Delta_{j} \\
& =\int \chi(x)\left\langle\left(\tilde{f}(t), e^{i x t} \widetilde{\omega}(y t)\right), \psi\right\rangle d x
\end{aligned}
$$

Сравнивая (3.11) и (3.12), получаем равенство (3.9), в котором слева стоит элемент пространства $\mathbb{B}$. Следовательно, и элемент, стоящий справа,

$$
\left(\tilde{f}(t), \int \chi(x) e^{i x t} \widetilde{\omega}(y t) d x\right)=(\tilde{f}(t), \widetilde{\chi}(t) \widetilde{\omega}(y t))
$$


также принадлежит пространству $\mathbb{B}$. Здесь $\widetilde{\chi}(t)=F[\chi(x)](t) \in Z_{d}$. Точно так же покажем, что интеграл по $y$ можно вынести за знак функционала. Из соотношений (3.7) получим

$$
\begin{aligned}
\int \chi(x) \int_{0}^{\beta} y^{s} L_{f}^{\omega}(x, y) d y d x & =\int_{0}^{\beta} y^{s} \int \chi(x)\left(\tilde{f}(t), e^{i x t} \widetilde{\omega}(y t)\right) d x d y \\
& =\int_{0}^{\beta} y^{s}(\tilde{f}(t), \widetilde{\chi}(t) \widetilde{\omega}(y t)) d y \\
& =\left(\tilde{f}(t), \widetilde{\chi}(t) \int_{0}^{\beta} y^{s} \widetilde{\omega}(y t) d y\right) .
\end{aligned}
$$

Отсюда и из (3.6) имеем

$$
\left\|\left(\tilde{f}(t), \widetilde{\chi}(t) \int_{0}^{\beta} y^{s} \widetilde{\omega}(y t) d y\right)\right\|_{\mathbb{B}} \leqslant C \underset{|x|<d}{\operatorname{ess} \sup } \hat{\mathbb{J}}\left(y^{b}\left\|L_{f}^{\omega}(x, y)\right\|_{\mathbb{B}}\right) \int_{-d}^{d}|\chi(x)| d x .
$$

Отметим, что

$$
\int_{0}^{\beta} y^{s} \widetilde{\omega}(y t) d y=\beta^{s+1} \widetilde{\eta}(\beta t), \quad \widetilde{\eta}(\zeta)=\frac{1}{\zeta^{s+1}} \int_{0}^{\zeta} \xi^{s} \widetilde{\omega}(\xi) d \xi
$$

и положим $\widetilde{\eta}_{\beta}(t)=\frac{1}{t^{n+1}} \beta^{s+1} \widetilde{\eta}(t \beta)$. Таким образом, соотношение (3.13) можно переписать в виде

$$
\left\|\left(\tilde{f}(t), \widetilde{\chi}(t) t^{n+1} \widetilde{\eta}_{\beta}(t)\right)\right\|_{\mathbb{B}} \leqslant C \underset{|x|<d}{\operatorname{ess} \sup } \hat{\mathbb{J}}\left(y^{b}\left\|L_{f}^{\omega}(x, y)\right\|_{\mathbb{B}}\right) \int_{-d}^{d}|\chi(x)| d x .
$$

Покажем, что семейство функций $\left\{\widetilde{\eta}_{\beta}(t), t=\sigma+i \tau \in \mathbb{C}, 0<\beta \leqslant 1\right\}$ удовлетворяет всем условиям леммы П.1 о короне (см. приложение).

Действительно, так как $\widetilde{\omega}(\xi) \in Z_{n} \backslash_{n+1} Z_{\gamma}$, то $\widetilde{\omega}(\xi)=\xi^{n+1} \widetilde{\omega}_{1}(\xi)$, где $\widetilde{\omega}_{1}(0) \neq 0$, и выполнены оценки

$$
|\widetilde{\omega}(\zeta)| \leqslant C_{N} e^{\gamma|\operatorname{Im} \zeta|}(1+|\operatorname{Re} \zeta|)^{-N}, \quad N=0,1, \ldots
$$

Отсюда и из (3.14) вытекает, что для всех $0<\beta \leqslant 1$ функции $\widetilde{\eta}_{\beta}(t)$ - целые аналитические, $\widetilde{\eta}_{\beta}(0) \neq 0$ и выполнены оценки

$$
\begin{aligned}
\left|\widetilde{\eta}_{\beta}(t)\right| & \leqslant \frac{\beta^{s+1}}{|t|^{n+1}} \frac{1}{\beta^{s+1}|t|^{s+1}}\left|\int_{0}^{t \beta} \zeta^{s} \widetilde{\omega}(\zeta) d \zeta\right| \leqslant C_{\varepsilon} e^{(\gamma+\varepsilon) \beta|\tau|}(1+\beta|\sigma|)^{Q_{2}} \\
& \leqslant C_{\varepsilon}^{1} e^{(\gamma+\varepsilon)|\tau|}(1+|\sigma|)^{Q_{2}}
\end{aligned}
$$

с некоторыми $C_{\varepsilon}^{1}$ и $Q_{2}$, не зависящими от $0<\beta \leqslant 1$. Покажем, что выполняется также и приведенная в приложении оценка (П.2). Пусть $|t| \geqslant 1$; тогда

$$
\sup _{0<\beta \leqslant 1}\left|\widetilde{\eta}_{\beta}(t)\right| \geqslant\left|\widetilde{\eta}_{\frac{1}{(1+\delta)|t|}}(t)\right|=\frac{1}{[(1+\delta)|t|]^{s+1}}\left|\widetilde{\eta}\left(\frac{1}{1+\delta} \frac{t}{|t|}\right)\right| \geqslant \frac{C}{|t|^{s+1}},
$$


так как всегда можно выбрать $\delta$ таким, чтобы аналитическая функция $\widetilde{\eta}(t)$ не обращалась в нуль на окружности радиуса $\frac{1}{1+\delta}$. Если теперь $|t|<1$, то всегда можно выбрать $\beta_{1}$ и $\beta_{2}$ так, чтобы $\widetilde{\eta}\left(\beta_{1} t\right)$ и $\widetilde{\eta}\left(\beta_{2} t\right)$ не имели обших нулей внутри еденичного круга. Поэтому

$$
\sup _{0<\beta \leqslant 1}\left|\widetilde{\eta}_{\beta}(t)\right| \geqslant \text { const }>0, \quad|t|<1 .
$$

Из (3.18) и (3.19) вытекает (П.2).

По лемме П.1 сушествуют счетное множество индексов $I$, числа $\lambda_{\beta}>0, \beta \in I$, $\sum_{\beta \in I} \lambda_{\beta}=1$, и целые аналитические функции $\left\{g_{\beta}(t), \beta \in I\right\}$ такие, что

$$
\left|g_{\beta}(t)\right| \leqslant A e^{a_{2}|\tau|}\left(1+|\sigma|^{Q}\right), \quad t \in \mathbb{C},
$$

для некоторых $A, a_{2}, Q$ и

$$
\sum_{\beta \in I} \lambda_{\beta} g_{\beta}(t) \widetilde{\eta}_{\beta}(t)=1, \quad t \in \mathbb{C}
$$

Пусть $\psi(\xi) \in \stackrel{D}{\mathscr{D}}_{a} ;$ тогда $\widetilde{\psi}(t) \in Z_{n}$ и $\widetilde{\psi}(t)=t^{n+1} \widetilde{\psi}_{1}(t)$, где $\widetilde{\psi}_{1} \in Z_{a}$. Из $(3.21)$ следует, что

$$
\widetilde{\psi}(t)=\sum_{\beta \in I} \lambda_{\beta} g_{\beta}(t) \widetilde{\eta}_{\beta}(t) \widetilde{\psi}(t)=\sum_{\beta \in I} \lambda_{\beta} g_{\beta}(t) \widetilde{\psi}_{1}(t) t^{n+1} \widetilde{\eta}_{\beta}(t)
$$

В силу оценок (3.17) и (3.20) множество $\left\{g_{\beta}(t) \widetilde{\psi}_{1}(t) t^{n+1} \widetilde{\eta}_{\beta}(t), \beta \in I\right\}$ ограничено в $Z_{\gamma+a_{2}+\varepsilon+a}$, следовательно, и в $Z$, поэтому ряд

$$
\sum_{\beta \in I} \lambda_{\beta}\left(\tilde{f}(t), g_{\beta}(t) \widetilde{\psi}_{1}(t) t^{n+1} \widetilde{\eta}_{\beta}(t)\right)
$$

сходится в $\mathscr{L}$ к $(\tilde{f}(t), \widetilde{\psi}(t))$. Покажем, что он сходится в $\mathbb{B}$, откуда, в частности, будет следовать, что $(f(\xi), \psi(\xi)) \in \mathbb{B}$. Для этого достаточно показать, что множество $\left\{\left(\tilde{f}(t), g_{\beta}(t) \widetilde{\psi}_{1}(t) t^{n+1} \widetilde{\eta}_{\beta}(t)\right), \beta \in I\right\}$ ограничено в $\mathbb{B}$. Согласно соотношению $(3.15)$, в котором $\widetilde{\chi}(t)=g_{\beta}(t) \widetilde{\psi}_{1}(t)$, а $d$ выбрано равным $a_{1}=\gamma+a_{2}+\varepsilon+a$, имеем

$$
\begin{aligned}
\left\|\left(\tilde{f}(t), g_{\beta}(t) \widetilde{\psi}_{1}(t) t^{n+1} \widetilde{\eta}_{\beta}(t)\right)\right\|_{\mathbb{B}} \\
\leqslant \underset{|x|<a_{1}}{\operatorname{ess} \sup _{\mathbb{\mathbb { J }}}}\left(y^{b}\left\|L_{f}^{\omega}(x, y)\right\|_{\mathbb{B}}\right) \int\left|F^{-1}\left[g_{\beta}(t) \widetilde{\psi}_{1}(t)\right](\xi)\right| d \xi \\
\leqslant C_{1} \underset{|x|<a_{1}}{\operatorname{ess} \sup _{1} \hat{\mathbb{J}}\left(y^{b}\left\|L_{f}^{\omega}(x, y)\right\|_{\mathbb{B}}\right) \mathscr{P}_{N_{1}}\left[\psi_{1}(\xi)\right]} \\
\leqslant \underset{|x|<a_{1}}{C_{2}} \operatorname{essup}_{\mid x \sup } \hat{\mathbb{J}}\left(y^{b}\left\|L_{f}^{\omega}(x, y)\right\|_{\mathbb{B}}\right) \mathscr{P}_{N}[\psi(\xi)] .
\end{aligned}
$$


Мы учли $(3.20)$ и тот факт, что $\mathscr{P}_{N_{1}}\left[\psi_{1}(\xi)\right] \leqslant C \mathscr{P}_{N}[\psi(\xi)]$ при достаточно большом $N$. Таким образом,

$$
(f(\xi), \psi(\xi))=\sum_{\beta \in I} \lambda_{\beta}\left(\tilde{f}(t), g_{\beta}(t) \widetilde{\psi}_{1}(t) t^{n+1} \widetilde{\eta}_{\beta}(t)\right),
$$

где ряд справа сходится в $\mathbb{B}$. Отсюда и из оценки (3.23) следует (3.4).

Пусть теперь $n \geqslant 0$ и нашлась функция $\omega_{0}(\xi) \in \mathscr{D}$, удовлетворяющая условиям теоремы; тогда, рассуждая, как и ранее, имеем

$$
\begin{aligned}
& \left\|\int \chi(x) L_{f}^{\omega_{0}}(x, 1) d x\right\|_{\mathbb{B}}=\left\|\left(\tilde{f}(t), \widetilde{\chi}(t) \widetilde{\omega}_{0}(t)\right)\right\|_{\mathbb{B}} \\
& \leqslant \underset{|x|<d}{\operatorname{ess} \sup _{\mid}}\left\|L_{f}^{\omega_{0}}(x, 1)\right\|_{\mathbb{B}} \int|\chi(x)| d x .
\end{aligned}
$$

Поскольку $\widetilde{\omega}_{0}(0) \neq 0$, очевидно, что семейство функций $\left\{t^{n+1} \widetilde{\eta}_{\beta}(t), \beta \in I\right\} \cup$ $\left\{\widetilde{\omega}_{0}(t)\right\}$ удовлетворяет всем условиям леммы П.1. Поэтому существуют числа $\nu_{\beta}>0, \mu>0$ и функции $g_{\beta}^{1}(t), g_{0}^{1}(t)$ с надлежашими свойствами такие, что

$$
1=\sum_{\beta \in I} \nu_{\beta} g_{\beta}^{1}(t) t^{n+1} \widetilde{\eta}_{\beta}(t)+\mu \widetilde{\omega}_{0}(t) g_{0}^{1}(t)
$$

Поэтому для любой $\psi \in \mathscr{D}$ имеем

$$
\widetilde{\psi}(t)=\sum_{\beta \in I} \nu_{\beta} g_{\beta}^{1}(t) \widetilde{\psi}(t) t^{n+1} \widetilde{\eta}_{\beta}(t)+\mu \widetilde{\omega}_{0}(t) g_{0}^{1}(t) \widetilde{\psi}(t)
$$

Теперь, используя оценки (3.23) и (3.25), аналогично предыдущему приходим к оценке (3.5). Теорема доказана.

Отметим, что условие измеримости в теореме 3.1 не является экзотическим. (Существуют простые примеры неизмеримых функций со значениями в банаховых пространствах.) Проверка этого условия иногда бывает достаточно затруднительной, поэтому в некоторых случаях полезны следуюшие замечания.

ЗАмЕчАнИЕ 3.1 . Пусть семейство банаховых пространств $\left\{\mathbb{B}_{\varepsilon}, \varepsilon>0\right\}$ таково, что $\mathbb{B} \subset \mathbb{B}_{\varepsilon} \subset \mathscr{L}$, причем

$$
\mathbb{B}=\left\{\eta \in \bigcap_{\varepsilon>0} \mathbb{B}_{\varepsilon}: \sup _{\varepsilon>0}\|\eta\|_{\mathbb{B}_{\varepsilon}}<\infty\right\}, \quad \text { кроме того, } \quad\|\eta\|_{\mathbb{B}_{\varepsilon}} \nearrow\|\eta\|_{\mathbb{B}}, \quad \varepsilon \rightarrow+0 .
$$

Тогда теорема 3.1 останется верной, если вместо измеримости функций $L_{f}^{\omega}(x, y)$ и $L_{f}^{\omega_{0}}(x, 1)$ как функций со значениями в $\mathbb{B}$ требовать измеримости этих функций как функций со значениями в $\mathbb{B}_{\varepsilon}$ при любых $\varepsilon>0$. 
Действительно, так как

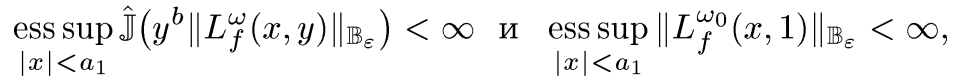

то, пользуясь теоремой 3.1 , для любых $\varepsilon>0$ и $\varphi \in \stackrel{n}{\mathscr{D}} \underset{a}{ }$ получаем

$$
\begin{aligned}
& \|(f(\xi), \varphi(\xi))\|_{\mathbb{B}_{\varepsilon}} \leqslant \\
& \leqslant C\left[\operatorname{ess} \sup _{|x|<a_{1}} \hat{\mathbb{J}}\left(y^{b}\left\|L_{f}^{\omega}(x, y)\right\|_{\mathbb{B}_{\varepsilon}}\right)+\underset{|x|<a}{\operatorname{ess} \sup _{f}}\left\|L_{f}^{\omega_{0}}(x, 1)\right\|_{\mathbb{B}_{\varepsilon}}\right] \mathscr{P}_{N}[\varphi] .
\end{aligned}
$$

Переходя здесь к пределу при $\varepsilon \rightarrow 0$ и учитьвая, что $a_{1}, C$ и $N$ не зависят от банаховых пространств $\mathbb{B}_{\varepsilon}$, получим (3.5).

ЗАмЕчаниЕ 3.2 . Если в условиях теоремы $3.1 \mathbb{B}=A^{*}$, где $A$ - сепарабельное банахово пространство, то условие (сильной) измеримости $L_{f}^{\omega}(x, y)$ и $L_{f}^{\omega_{0}}(x, 1)$ можно заменить условием *-слабой измеримости (см. $\S 1$ ). При этом в доказательстве вместо (сильного) интеграла Бохнера следует пользоваться *-слабым интегралом Бохнера.

Приведем теперь формулировки аналогичных теорем для пространств $E^{\uparrow}, E^{\downarrow}$ и $\mathscr{S}$.

ТЕОРемА 3.1'. Пусть $\mathbb{J}-$ автомодельный функиионал, $\mathbb{B}$ - банахово и $\mathscr{L}$ локально выпуклое пространства, причем $\mathbb{B} \subset \mathscr{L}$ линейно и непрерывно. Пусть $f \in \mathbb{L}\left(E^{\uparrow} \rightarrow \mathscr{L}\right)$ и для некоторой $\omega(\xi) \in \stackrel{n}{E^{\uparrow}} \backslash^{n+1} \stackrel{1}{E}^{\uparrow}$ выполнено условие: $L_{f}^{\omega}(x, y)$ - измеримая при $(x, y) \in \mathbb{R}^{1} \times(0,1]$ функиия со значениями в $\mathbb{B}$, причем для любого $\varepsilon>0$ найдется $b$ такое, что

$$
\underset{x \in \mathbb{R}}{\operatorname{ess} \sup ^{-\varepsilon|x|} \hat{\mathbb{J}}\left(y^{b}\left\|L_{f}^{\omega}(x, y)\right\|_{\mathbb{B}}\right)<\infty .}
$$

Тогда

$$
(f(\xi), \varphi(\xi)) \in \mathbb{B} \quad \forall \varphi \in \stackrel{n}{E}^{\uparrow} .
$$

Более того, для любого $\varepsilon>0$ существует $\varepsilon_{1}$ такое, что для любого $b$ найдутся $C$ и $N$ такие, ито

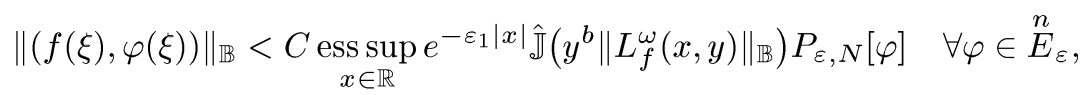

где $\varepsilon_{1}, C$ и $N$ не зависят от $f, \varphi$ и $\mathbb{B}$.

Если $n \geqslant 0$ и для некоторой основной функции $\omega_{0} \in E^{\uparrow}$ такой, что $\int \omega_{0}(\xi) d \xi \neq 0$, выполнено следующее: $L_{f}^{\omega_{0}}(x, 1)$ - измеримая функция по $x$ со значениями в $\mathbb{B}$ и для любого $\varepsilon>0$

$$
\underset{x \in \mathbb{R}}{\operatorname{ess} \sup ^{-\varepsilon|x|}}\left\|L_{f}^{\omega_{0}}(x, 1)\right\|_{\mathbb{B}}<\infty,
$$

mо $f \in \mathbb{L}\left(E^{\uparrow} \rightarrow \mathbb{B}\right)$. Более того, для любого $\varepsilon>0$ существует $\varepsilon_{1}$ такое, что для любого $b$ найдутся $C$ и $N$ такие, что

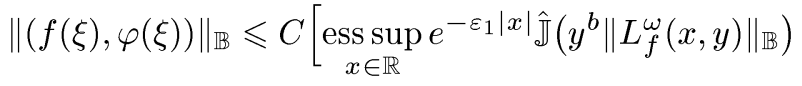

$$
\begin{aligned}
& \left.+\underset{x \in \mathbb{R}}{\operatorname{ess} \sup ^{-\varepsilon_{1}|x|}}\left\|L_{f}^{\omega_{0}}(x, 1)\right\|_{\mathbb{B}}\right] P_{\varepsilon, N}[\varphi] \quad \forall \varphi \in E_{\varepsilon},
\end{aligned}
$$

әде $\varepsilon_{1}, C$ и $N$ не зависят от $f, \varphi$ и $\mathbb{B}$. 
ТЕОРема $3.1^{\prime \prime}$. Пусть $\mathbb{J}$ - автомодельный функиионал, $\mathbb{B}$ - банахово и $\mathscr{L}-$ локально выпуклое пространства, причем $\mathbb{B} \subset \mathscr{L}$ линейно и непрерывно. Пусть $f \in \mathbb{L}\left(E^{\downarrow} \rightarrow \mathscr{L}\right)$ и для некоторой $\omega(\xi) \in \stackrel{n}{E^{\downarrow} \backslash \stackrel{n+1}{E} \downarrow}$ выполнено условие: $L_{f}^{\omega}(x, y)$ - измеримая при $(x, y) \in \mathbb{R}^{1} \times(0,1]$ функция со значениями в $\mathbb{B}$, причем существуют $d$ и $\ell$ такие, что

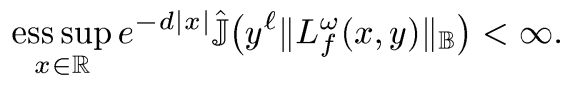

Тогда $(f(\xi), \varphi(\xi)) \in \mathbb{B} \quad \forall \varphi \in \stackrel{n}{E} \downarrow$.

Более того, для любих а и $b$ существуют $a_{1}, C$ и $N$ такие, что

$$
\|(f(\xi), \varphi(\xi))\|_{\mathbb{B}}<C \underset{x \in \mathbb{R}}{\operatorname{ess} \sup } e^{-a|x| \hat{\mathbb{J}}}\left(y^{b}\left\|L_{f}^{\omega}(x, y)\right\|_{\mathbb{B}}\right) P_{a_{1}, N}[\varphi] \quad \forall \varphi \in \stackrel{n}{E}^{\downarrow}
$$

где $a_{1}, C$ и $N$ не зависят от $f, \varphi$ и $\mathbb{B}$.

Если $n \geqslant 0$ и для некоторой основной функции $\omega_{0} \in E^{\downarrow}$ такой, что $\int \omega_{0}(\xi) d \xi \neq 0$, выполнено: $L_{f}^{\omega_{0}}(x, 1)$ - измеримая функция по $x$ со значениями в $\mathbb{B}$ и существует а такое, что

$$
\underset{x \in \mathbb{R}}{\operatorname{ess} \sup ^{-a|x|}}\left\|L_{f}^{\omega_{0}}(x, 1)\right\|_{\mathbb{B}}<\infty
$$

mо $f \in \mathbb{L}\left(E^{\downarrow} \rightarrow \mathbb{B}\right)$. Более того, для любьх а и такие, что

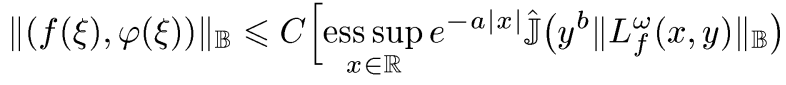

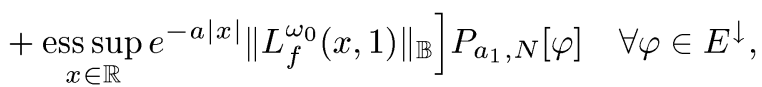

где $a_{1}, C$ и $N$ не зависят от $f, \varphi$ и $\mathbb{B}$.

ТЕОРемА $3.1^{\prime \prime \prime}$. Пусть $\mathbb{J}$ - автомодельный функиионал, $\mathbb{B}$ - банахово и $\mathscr{L}$ локально выпуклое пространства, причем $\mathbb{B} \subset \mathscr{L}$ линейно и непрерывно, $f \in \mathbb{L}(\mathscr{S} \rightarrow \mathscr{L}), \omega(\xi) \in \stackrel{n}{\mathscr{S}} \backslash^{n+1} \mathscr{S}$, и пусть $L_{f}^{\omega}(x, y)-$ измеримая $п р и(x, y) \in \mathbb{R}^{1} \times$ $(0,1]$ функция со значениями в $\mathbb{B}$, причем существуют $d$ и $\ell$ такие, что

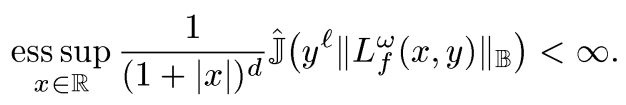

Тогда $(f(\xi), \varphi(\xi)) \in \mathbb{B} \forall \varphi \in \stackrel{n}{\mathscr{S}}$ и для любых а и в существуют $N$ и $C$ такие что

$$
\|(f(\xi), \varphi(\xi))\|_{\mathbb{B}}<C \operatorname{ess}_{x \in \mathbb{R}} \frac{1}{(1+|x|)^{a}} \hat{\mathbb{J}}\left(y^{b}\left\|L_{f}^{\omega}(x, y)\right\|_{\mathbb{B}}\right) \mathscr{P}_{N}[\varphi] \quad \forall \varphi \in \stackrel{n}{\mathscr{S}}
$$


причем $C$ и не зависят от $f, \varphi$ и $\mathbb{B}$.

Если $n \geqslant 0$ и найдется функиия $\omega_{0} \in \mathscr{S} \backslash \stackrel{0}{\mathscr{S}}$ такая, ито $L_{f}^{\omega_{0}}(x, 1)-$ измеримая функиия по $x$ со значениями в $\mathbb{B}$ и для некоторого $d$

$$
\operatorname{ess}_{x \in \mathbb{R}} \frac{1}{(1+|x|)^{d}}\left\|L_{f}^{\omega_{0}}(x, 1)\right\|_{\mathbb{B}}<\infty
$$

mо $f \in \mathbb{L}(\mathscr{S} \rightarrow \mathbb{B})$. Более того, для любьх а и ь существуют $C$ и $N$ такие, чmo

$$
\begin{aligned}
& \|(f(\xi), \varphi(\xi))\|_{\mathbb{B}} \leqslant C\left[\operatorname{ess}_{x \in \mathbb{R}} \frac{1}{(1+|x|)^{a}} \hat{\mathbb{J}}\left(y^{b}\left\|L_{f}^{\omega}(x, y)\right\|_{\mathbb{B}}\right)\right. \\
& \left.+\operatorname{ess}_{x \in \mathbb{R}} \frac{1}{(1+|x|)^{a}}\left\|L_{f}^{\omega_{0}}(x, 1)\right\|_{\mathbb{B}}\right] \mathscr{P}_{N}[\varphi] \quad \forall \varphi \in \mathscr{S},
\end{aligned}
$$

причем $C$ и $N$ не зависят от $f, \varphi$ и $\mathbb{B}$.

Теоремы $3.1^{\prime}-3.1^{\prime \prime \prime}$ доказываются аналогично теореме 3.1 , только надо воспользоваться соответствующими общими оценками класса для соответствующих пространств (см. (2.12)-(2.14)) и леммами П.2-П.4 (см. приложение). Замечания 3.1, 3.2 остаются справедливыми и для этих теорем.

Для дальнейшего нам понадобятся несколько лемм.

Лемма 3.1. Пусть $\mathbb{B}$ - банахово пространство и обобщенная функиия $f \in$ $\mathbb{L}(\mathscr{D} \rightarrow \mathbb{B})$ квазиасимптотически ограничена относительно автомодельного функционала $\mathbb{J}$ на $\stackrel{\ell}{\mathscr{D}}$ для некоторого щелого $\ell$. Другими словами,

$$
\mathbb{J}\left(\left\|\left(f\left(\frac{\xi}{k}\right), \varphi(\xi)\right)\right\|_{\mathbb{B}}\right)<\infty \quad \forall \varphi \in \stackrel{\ell}{\mathscr{D}}
$$

Тогда для любого а $>0$ существуют $C$ и $N$ такие, что

$$
\mathbb{J}\left(\left\|\left(f\left(\frac{\xi}{k}\right), \varphi(\xi)\right)\right\|_{\mathbb{B}}\right) \leqslant C \mathscr{P}_{N}[\varphi(\xi)] \quad \forall \varphi \in \stackrel{\ell}{\mathscr{D}}_{a}
$$

(Напомним, что если $\ell<0$, то $\stackrel{\ell}{\mathscr{D}}=\mathscr{D}$.

ДокАЗАТЕЛЬСТВО. По аксиоме 3$)$ для любой $\varphi$ из $\stackrel{\ell}{\mathscr{D}}$ имеем

$$
\mathbb{J}\left(\left\|\left(f\left(\frac{\xi}{k}\right), \varphi(\xi)\right)\right\|_{\mathbb{B}}\right)=\lim _{\varkappa \rightarrow+\infty} \mathbb{J}\left(\chi_{[1, \varkappa]}(k)\left\|\left(f\left(\frac{\xi}{k}\right), \varphi(\xi)\right)\right\|_{\mathbb{B}}\right) .
$$

Выражение, стояшее справа, есть предел монотонно возрастающей последовательности непрерывных выпуклых функционалов на $\stackrel{\ell}{\mathscr{D}}$. Действительно, их выпуклость 
очевидна, а их непрерывность означает непрерывность на любом $\stackrel{\ell}{\mathscr{D}} a, a>0$. Замечая, что $\varphi(k \xi) \in \stackrel{\ell}{\mathscr{D}}_{a}$, если $\varphi(\xi) \in \stackrel{\ell}{\mathscr{D}}_{a}$ и $k>1$, и пользуясь леммой 2.3, для $\varphi \in \stackrel{\ell}{\mathscr{D}}_{a}$ имеем

$$
\begin{aligned}
\mathbb{J}\left(\chi_{[1, \varkappa]}(k) \|\left(f\left(\frac{\xi}{k}\right), \varphi(\xi)\right)\right. & \left.\|_{\mathbb{B}}\right) \\
& \leqslant C_{\varepsilon} \sup _{1 \leqslant k \leqslant \varkappa} \frac{1}{k^{\alpha-\varepsilon}}\left\|\left(f\left(\frac{\xi}{k}\right), \varphi(\xi)\right)\right\|_{\mathbb{B}} \\
& \leqslant C_{\varepsilon} \sup _{1 \leqslant k \leqslant \varkappa} \frac{k}{k^{\alpha-\varepsilon}}\|(f(\xi), \varphi(k \xi))\|_{\mathbb{B}} \leqslant C_{\varepsilon}^{1} \sup _{1 \leqslant k \leqslant \varkappa} \frac{k}{k^{\alpha-\varepsilon}} \mathscr{P}_{N}[\varphi(k \xi)] \\
& =C_{\varepsilon}^{2} \sup _{1 \leqslant k \leqslant \varkappa} \frac{k^{2 N+2}}{k^{\alpha-\varepsilon}} \mathscr{P}_{N}[\varphi(\xi)] \leqslant C_{\varepsilon, \varkappa}^{1} \mathscr{P}_{N}[\varphi],
\end{aligned}
$$

где $N$ зависит от $a$. Таким образом, функционал, стоящий слева в (3.34), непрерывен снизу на $\stackrel{\ell}{\mathscr{D}}$. В силу бочечности $\stackrel{\ell}{\mathscr{D}}$ он будет непрерывен на $\stackrel{\ell}{\mathscr{D}}$. Отсюда и следует (3.33).

СлЕДСТВИЕ 3.1. В условиях лем.мы для любых а и $a_{1}$ существуют $A, N u$ b такие, что

$$
\sup _{\substack{|x|<a_{1} \\ 0<y \leqslant 1}} y^{b} \mathbb{J}\left(\left\|L_{f}^{\varphi}\left(\frac{x}{k}, \frac{y}{k}\right)\right\|_{\mathbb{B}}\right) \leqslant A \mathscr{P}_{N}[\varphi] \quad \forall \varphi \in \stackrel{\ell}{\mathscr{D}}_{a} .
$$

Действительно, фиксируя $a$ и $a_{1}$ и подставляя в (3.33) функцию $\frac{1}{y} \varphi\left(\frac{x-\xi}{y}\right)$, где $\operatorname{supp} \varphi(\xi) \subset[-a, a]$, имеем

$$
\begin{aligned}
\mathbb{J}\left(\left\|L_{f}^{\varphi}\left(\frac{x}{k}, \frac{y}{k}\right)\right\|_{\mathbb{B}}\right) & =\mathbb{J}\left(\left\|\left(f\left(\frac{\xi}{k}\right), \frac{1}{y} \omega\left(\frac{x-\xi}{y}\right)\right)\right\|_{\mathbb{B}}\right) \\
& \leqslant C \mathscr{P}_{N}\left[\frac{1}{y} \varphi\left(\frac{x-\xi}{y}\right)\right] \leqslant \frac{A}{y^{b}} \mathscr{P}_{N}[\varphi(\xi)] \forall \varphi \in \stackrel{\mathscr{D}}{a}_{a}, \quad|x|<a_{1},
\end{aligned}
$$

что и требовалось доказать.

ЗАмЕчАнИЕ 3.3. Лемма 3.1 и следствие 3.1 останутся справедливыми, если в (3.32) и (3.33) заменить $k$ на $\frac{1}{k}$.

ЗАмечАнИЕ 3.4. Лемма 3.1, следствие 3.1 и замечание 3.3 останутся справедливыми, если вместо пространства $\mathscr{D}$ рассматривать пространства $E^{\uparrow}, E^{\downarrow}$ или $\mathscr{S}$, следует только в оценках (3.33) и следствии заменить правую часть на соответствующую общую оценку класса (см. 1 1). 
ЛЕмма 3.2. Пусть $\mathbb{B}$ - банахово пространство и обобщенная функиия $\tilde{g}(t)$ принадлежит $\mathbb{L}(Z \rightarrow \mathbb{B})$. Тогда для любого иелого $q \geqslant 0$ справедливо соотношение

$$
\begin{aligned}
& k(\tilde{g}(k t), \tilde{\varphi}(t))= \\
& \quad=\sum_{j=0}^{q}(-1)^{j}\left(\tilde{g}(t), t^{j} \widetilde{\varphi}^{(j)}(t)\right) \int_{1}^{k} \frac{1}{k_{1}} \frac{d k_{1}}{k_{1}} \int_{1}^{k_{1}} \cdots \frac{1}{k_{j-1}} \frac{d k_{j-1}}{k_{j-1}} \int_{1}^{k_{j-1}} \frac{1}{k_{j}} \frac{d k_{j}}{k_{j}} \\
& \quad+(-1)^{q+1} \int_{1}^{k} \frac{1}{k_{1}} \frac{d k_{1}}{k_{1}} \int_{1}^{k_{1}} \cdots \frac{1}{k_{q}} \frac{d k_{q}}{k_{q}} \int_{1}^{k_{q}} \frac{d \xi}{\xi} \xi^{q+1}\left(\tilde{g}(t \xi), t^{q+1} \tilde{\varphi}^{(q+1)}(t)\right) .
\end{aligned}
$$

Если $\mathbb{J}(\cdot)$ - автомодельный функционал порядка $\alpha>0$, то

$$
\begin{aligned}
& \mathbb{J}\left(k\|(\tilde{g}(k t), \widetilde{\varphi}(t))\|_{\mathbb{B}}\right) \leqslant \sum_{j=0}^{q} C_{j} \cdot \mathbb{J}\left(\frac{1}{k^{j}}\right) \cdot\left\|\left(t^{j} \tilde{g}(t), \widetilde{\varphi}^{(j)}(t)\right)\right\|_{\mathbb{B}} \\
& \quad+C_{q+1} \cdot \mathbb{J}\left(\frac{1}{k^{q+1}} k\left\|\left((k t)^{q+1} \tilde{g}(k t),\left(\frac{d}{d t}\right)^{q+1} \widetilde{\varphi}(t)\right)\right\|_{\mathbb{B}}\right) \quad \forall \widetilde{\varphi} \in Z .
\end{aligned}
$$

Здесь $C_{j}$ - постоянные, зависящие только от $\mathbb{J}$, причем $C_{0}=1$.

ДокАЗАтЕЛЬСтво. Положим $t \tilde{g}(t)=\tilde{f}(t) \in \mathbb{L}(Z \rightarrow \mathbb{B})$. Сперва заметим, что

$$
\begin{aligned}
\frac{1}{k} \int_{1}^{k}\left(\tilde{f}(\varkappa t), \widetilde{\varphi}^{\prime}(t)\right) \frac{d \varkappa}{\varkappa} & =\frac{1}{k}\left(\tilde{f}(t), \int_{1}^{k} \widetilde{\varphi}^{\prime}\left(\frac{t}{\varkappa}\right) \frac{d \varkappa}{\varkappa^{2}}\right)=\frac{1}{k}\left(\tilde{f}(t), \int_{1 / k}^{1} \widetilde{\varphi}^{\prime}(t \xi) d \xi\right) \\
& =\frac{1}{k}\left(\tilde{f}(t), \frac{1}{t} \int_{t / k}^{t} \widetilde{\varphi}^{\prime}(\xi) d \xi\right)=\frac{1}{k}\left(\tilde{f}(t), \frac{\tilde{\varphi}(t)-\tilde{\varphi}\left(\frac{t}{k}\right)}{t}\right) \\
& =\frac{1}{k}(\tilde{g}(t), \tilde{\varphi}(t))-\frac{1}{k}\left(\tilde{g}(t), \tilde{\varphi}\left(\frac{t}{k}\right)\right),
\end{aligned}
$$

откуда имеем

$$
k(\tilde{g}(k t), \widetilde{\varphi}(t))=(\tilde{g}(t), \widetilde{\varphi}(t))-\int_{1}^{k} \frac{1}{\varkappa} \frac{d \varkappa}{\varkappa} \varkappa\left((t \varkappa) \tilde{g}(t \varkappa), \widetilde{\varphi}^{\prime}(t)\right) .
$$

Применяя эту формулу к подынтегральному выражению (для функций $t \tilde{g}(t)$ и $\left.\widetilde{\varphi}^{\prime}(t)\right)$, получим

$$
\begin{aligned}
k(\tilde{g}(k t), \tilde{\varphi}(t))= & (\tilde{g}(t), \widetilde{\varphi}(t))-\left(t \tilde{g}(t), \widetilde{\varphi}^{\prime}(t)\right) \int_{1}^{k} \frac{1}{k_{1}} \frac{d k_{1}}{k_{1}} \\
& +\int_{1}^{k} \frac{1}{k_{1}} \frac{d k_{1}}{k_{1}} \int_{1}^{k_{1}} \frac{1}{k_{2}} \frac{d k_{2}}{k_{2}} k_{2}\left(\left(t k_{2}\right)^{2} \tilde{g}\left(t k_{2}\right), \widetilde{\varphi}^{\prime \prime}(t)\right) .
\end{aligned}
$$

Производя далее то же самое $q$ раз, получим (3.35).

Делая в (3.35) оценку по норме $\mathbb{B}$, применяя к обеим частям полученного неравенства функционал $\mathbb{J}(\cdot)$ и пользуясь неравенством Харди (сперва для $\mathbb{J}(\cdot)$, затем для $\mathbb{J}\left(\frac{1}{k} \cdot\right)$ и т.д.; см. $\left.(2.6)\right)$, получим (3.36). Лемма доказана. 
ЗАмЕЧАНИЕ 3.5. Лемма 3.2 останется справедливой, если вместо пространства $Z$ рассматривать пространства $\widetilde{E}^{\uparrow}, \widetilde{E}^{\downarrow}$ или $\mathscr{S}$.

Лемма 3.3. Пусть $\mathbb{B}$ - банахово пространство, $\mathbb{J}$ - автомодельный функционал порядка $\alpha, f(\xi) \in \mathbb{L}(\mathscr{D} \rightarrow \mathbb{B})$ и для некоторого $n$ нашлась функиия $\omega(\xi) \in \stackrel{n}{\mathscr{D} \backslash} \backslash \stackrel{n+1}{\mathscr{D}}$ такая, что для любого а >0 существует $d$ такое, что

$$
\sup _{\substack{|x|<a \\ 0<y \leqslant 1}} y^{d} \mathbb{J}\left(\left\|L_{f}^{\omega}\left(\frac{x}{k}, \frac{y}{k}\right)\right\|_{\mathbb{B}}\right)<\infty .
$$

Тогда обобщенная функиия $f(\xi)$ квазиасимптотически ограничена относительно $\mathbb{J}$ на $\stackrel{n}{\mathscr{D}}$, m.e.

$$
\mathbb{J}\left(\left\|\left(f\left(\frac{\xi}{k}\right), \varphi(\xi)\right)\right\|_{\mathbb{B}}\right)<\infty \quad \forall \varphi \in \stackrel{n}{\mathscr{D}} .
$$

Более того, для любого а существует $a_{1}$ такое, что для любого $b$ найдутся C и $N$ maкue, umo

$$
\mathbb{J}\left(\left\|\left(f\left(\frac{\xi}{k}\right), \varphi(\xi)\right)\right\|_{\mathbb{B}}\right) \leqslant C \sup _{\substack{|x|<a_{1} \\ 0<y \leqslant 1}} y^{b} \mathbb{J}\left(\left\|L_{f}^{\omega}\left(\frac{x}{k}, \frac{y}{k}\right)\right\|_{\mathbb{B}}\right) \mathscr{P}_{N}[\varphi] \quad \forall \varphi \in \mathscr{D}_{a} .
$$

Здесь числа $a_{1}, C$ и $N$ не зависят от $f$ и $\mathbb{B}$.

ДокАЗАтЕльство. Фиксируем $\varepsilon>0$. Рассмотрим множество измеримых на $(1,+\infty)$ функций $\eta(k)$ со значениями в $\mathbb{B}$, т.е. функций из $\mathscr{M}((1,+\infty) \rightarrow \mathbb{B})$, для которых $\mathbb{J}\left(\frac{1}{k^{\varepsilon}}\|\eta(k)\|_{\mathbb{B}}\right)<\infty$. Это множество есть банахово пространство с нормой $\|\eta\|_{H^{\varepsilon}}=\mathbb{J}\left(\frac{1}{k^{\varepsilon}}\|\eta(k)\|_{\mathbb{B}}\right)$ (см. замечание 2.1). Обозначим его $H^{\varepsilon}$. В качестве локально выпуклого пространства $\mathscr{L}$ рассмотрим пространство измеримых на $[1,+\infty)$ функций со значениями в $\mathbb{B}$ со счетным числом полунорм

$$
Q_{N}[\eta(k)]=\mathbb{J}\left(\chi_{[1, N]}(k)\|\eta(k)\|_{\mathbb{B}}\right), \quad N=2,3, \ldots
$$

Ясно, что $H^{\varepsilon} \subset \mathscr{L}$ линейно и непрерывно, и если $H=\{\eta(k) \in \mathscr{M}((1,+\infty) \rightarrow \mathbb{B})$ : $\left.\|\eta(k)\|_{H}=\mathbb{J}\left(\|\eta(k)\|_{\mathbb{B}}\right)<\infty\right\}$, то

$$
H=\left\{\eta \in \bigcap_{\varepsilon>0} H^{\varepsilon}: \sup _{\varepsilon}\|\eta\|_{H^{\varepsilon}}<\infty\right\}, \quad \text { кроме того, }\|\eta\|_{H^{\varepsilon}} \nearrow\|\eta\|_{H}, \quad \varepsilon \rightarrow+0
$$

(см. аксиому 3) автомодельных функционалов).

Рассмотрим обобщенную функцию $g(\xi) \in \mathbb{L}(\mathscr{D} \rightarrow \mathscr{L})$, сопоставляющую каждой $\varphi \in \mathscr{D}$ функцию $\eta(k) \in \mathscr{L}$, определяемую формулой

$$
\eta(k)=(g(\xi), \varphi(\xi))=\left(f\left(\frac{\xi}{k}\right), \varphi(\xi)\right)=k(f(\xi), \varphi(k \xi)) \quad \forall \varphi \in \mathscr{D}
$$


Очевидно, что функция $\eta(k)$ принадлежит $\mathscr{L}$, поскольку она является непрерьвной по $k$ со значениями в $\mathbb{B}$. Далее, имеем

$$
L_{g}^{\omega}(x, y)=\left(g(\xi), \frac{1}{y} \omega\left(\frac{x-\xi}{y}\right)\right)=k\left(f(\xi), \frac{1}{y} \omega\left(\frac{x-k \xi}{y}\right)\right)=L_{f}^{\omega}\left(\frac{x}{k}, \frac{y}{k}\right)
$$

В силу (3.37) для любого $a>0$ найдутся $A$ и $d$ такие, что

$$
\begin{aligned}
\left\|L_{g}^{\omega}(x, y)\right\|_{H^{\varepsilon}} & =\mathbb{J}\left(\frac{1}{k^{\varepsilon}}\left\|L_{f}^{\omega}\left(\frac{x}{k}, \frac{y}{k}\right)\right\|_{\mathbb{B}}\right) \leqslant \mathbb{J}\left(\left\|L_{f}^{\omega}\left(\frac{x}{k}, \frac{y}{k}\right)\right\|_{\mathbb{B}}\right) \\
& =\left\|L_{g}^{\omega}(x, y)\right\|_{H} \leqslant \frac{A}{y^{d}}
\end{aligned}
$$

при $|x|<a, 0<y \leqslant 1$, так что $L_{g}^{\omega}(x, y) \in H \subset H^{\varepsilon}$. Покажем, что функция $L_{g}^{\omega}(x, y)$ измерима по $(x, y)$ как функция со значениями в $H^{\varepsilon}$.

Действительно, из (3.40) следует, что $\psi_{n}(x, y)=\chi_{[1, n]}(k) L_{f}^{\omega}\left(\frac{x}{k}, \frac{y}{k}\right)$ непрерывна по $(x, y)$ как функция со значениями в $H^{\varepsilon}$ (ее носитель по $k$ компактен). Из соотношений

$$
\begin{aligned}
\left\|L_{g}^{\omega}(x, y)-\psi_{n}(x, y)\right\|_{H^{\varepsilon}} & =\mathbb{J}\left(\frac{1}{k^{\varepsilon}}\left\|\left(1-\chi_{[1, n]}(k)\right) L_{f}^{\omega}\left(\frac{x}{k}, \frac{y}{k}\right)\right\|_{\mathbb{B}}\right) \\
& \leqslant \frac{1}{n^{\varepsilon}} \mathbb{J}\left(\left|1-\chi_{[1, n]}(k)\right|\left\|L_{f}^{\omega}\left(\frac{x}{k}, \frac{y}{k}\right)\right\|_{\mathbb{B}}\right) \\
& \leqslant \frac{1}{n^{\varepsilon}} \mathbb{J}\left(\left\|L_{f}^{\omega}\left(\frac{x}{k}, \frac{y}{k}\right)\right\|_{\mathbb{B}}\right) \underset{n \rightarrow \infty}{\longrightarrow} 0
\end{aligned}
$$

вытекает, что функция $L_{g}^{\omega}(x, y)$ есть предел непрерывных функций со значениями в $H^{\varepsilon}$, а потому она измерима.

Из соотношений (3.41) имеем

$$
\sup _{|x|<a} \hat{\mathbb{J}}^{\infty}\left(y^{d}\left\|L_{g}^{\omega}(x, y)\right\|_{H}\right)<\infty
$$

где, напомним, $\hat{\mathbb{J}}^{\infty}(\cdot)=\operatorname{ess} \sup _{0<y \leqslant 1}(\cdot)$.

Замечая теперь, что выполнены все условия теоремы 3.1 (при $\mathbb{J}=\mathbb{J}^{\infty}$ и $\mathbb{B}=H$ ), и учитывая замечание 3.1 (где $\mathbb{B}_{\varepsilon}=H_{\varepsilon}$ ), заключаем, что $(g(\xi), \varphi(\xi)) \in H$ для любой $\varphi \in \stackrel{n}{\mathscr{D}}$, причем для любого $a>0$ существует $a_{1}$ такое, что для любого $b$ найдутся $C$ и $N$ такие, что

$$
\begin{aligned}
\|(g(\xi), \varphi(\xi))\|_{H} & =\left\|\left(f\left(\frac{\xi}{k}\right), \varphi(\xi)\right)\right\|_{H} \\
& \leqslant C \sup _{|x|<a_{1}} \hat{\mathfrak{J}}^{\infty}\left(y^{b}\left\|L_{g}^{\omega}(x, y)\right\|_{H}\right) \mathscr{P}_{N}[\varphi], \quad \varphi \in \stackrel{n}{D}_{a}
\end{aligned}
$$


(см. (3.4)), при этом $a_{1}, C$ и $N$ не зависят от $f$ и $\mathbb{B}$. Отсюда получаем, что для любого $a>0$ найдется $a_{1}$ такое, что для любого $b$ существуют $C_{1}$ и $N$ такие, что

$$
\begin{aligned}
\mathbb{J}\left(\left\|\left(f\left(\frac{\xi}{k}\right), \varphi(\xi)\right)\right\|_{\mathbb{B}}\right) & \leqslant C \sup _{|x|<a_{1}} \hat{\mathbb{J}}^{\infty}\left(y^{b} \mathbb{J}\left(\left\|L_{f}^{\omega}\left(\frac{x}{k}, \frac{y}{k}\right)\right\|_{\mathbb{B}}\right)\right) \mathscr{P}_{N}[\varphi] \\
& \leqslant C \sup _{|x|<a_{1}} \hat{\mathbb{J}}^{\infty}\left(y^{b} \frac{A}{y^{d}}\right) \mathscr{P}_{N}[\varphi] \leqslant A C \mathscr{P}_{N}[\varphi] \quad \forall \varphi \in \mathscr{D}_{a} .
\end{aligned}
$$

Здесь считаем, что $b>d$ и, следовательно, $\hat{J}^{\infty}\left(y^{b-d}\right)=1$. Кроме того, использована оценка (3.41). Неравенство (3.38), а вместе с ним и лемма доказаны.

ЗАмЕчАниЕ 3.6. Лемма 3.3 останется справедливой, если всюду в $L_{f}^{\omega}\left(\frac{x}{k}, \frac{y}{k}\right)$ и $f\left(\frac{\xi}{k}\right)$ заменить $k$ на $\frac{1}{k}$.

ЛЕмма 3.4. Пусть выполнены условия леммы $3.3 ;$ тогда

$$
\mathbb{J}\left(\left\|\left(f\left(\frac{\xi}{k}\right), \varphi(\xi)\right)\right\|_{\mathbb{B}}\right)<\infty \quad \forall \varphi \in \stackrel{m}{\mathscr{D}},
$$

əде $m=\min \{n,[-\alpha]\}$.

Более того, если $\omega_{0}(\xi) \in \mathscr{D} \backslash \stackrel{0}{\mathscr{D}}$, то для любого а $>0$ существует $a_{1}$ такое, что для любого $b$ найдутся $C$ и $N$ такие, что

$$
\begin{aligned}
\mathbb{J}\left(\|\left(f\left(\frac{\xi}{k}\right), \varphi(\xi)\right)\right. & \left.\|_{\mathbb{B}}\right) \leqslant C\left[\sup _{\substack{|x|<a_{1} \\
0<y \leqslant 1}} y^{b} \mathbb{J}\left(\left\|L_{f}^{\omega}\left(\frac{x}{k}, \frac{y}{k}\right)\right\|_{\mathbb{B}}\right)\right. \\
& \left.+\sup _{|x|<a_{1}} \hat{\mathbb{J}}\left(y^{b}\left\|L_{f}^{\omega}(x, y)\right\|_{\mathbb{B}}\right)+\sup _{|x|<a_{1}}\left\|L_{f}^{\omega_{0}}(x, 1)\right\|_{\mathbb{B}}\right] \mathscr{P}_{N}[\varphi] \quad \forall \varphi \in \stackrel{m}{\mathscr{D}}_{a}
\end{aligned}
$$

Здесь числа $a_{1}, C$ и $N$ не зависят от $f$ u $\mathbb{B}$.

ДоКАЗАТЕЛЬСТВО. Если $n<[-\alpha]+1$, то $m=n$ и наши утверждения следуют из леммы 3.3. Пусть $n \geqslant[-\alpha]+1$. Воспользуемся леммой 3.2. Для этого рассмотрим функционал $\mathbb{J}\left(\frac{1}{k^{m+1}} \cdot\right)$, его порядок равен $\beta=\alpha+[-\alpha]+1>0$ (см. лемму $2.2)$. Теперь можно воспользоваться соотношением (3.36) для этого функционала и для обобщенной функции $\tilde{g}(t)=t^{m+1} \tilde{f}(t)$. Пусть $\varphi(\xi)$ - произвольная функция

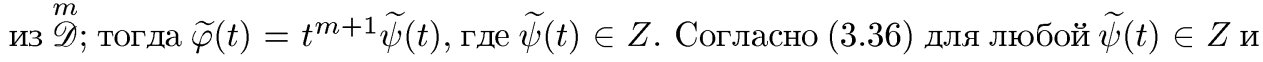
$q=n-m-1 \geqslant 0$ имеем

$$
\begin{aligned}
\mathbb{J}\left(\frac{1}{k^{m+1}} k\left\|\left((k t)^{m+1} \tilde{f}(k t), \widetilde{\psi}(t)\right)\right\|_{\mathbb{B}}\right) \\
\leqslant \sum_{j=0}^{n-m-1} C_{j} \mathbb{J}\left(\frac{1}{k^{m+1}} \frac{1}{k^{j}}\right)\left\|\left(t^{j+m+1} \tilde{f}(t), \widetilde{\psi}^{(j)}(t)\right)\right\|_{\mathbb{B}} \\
\quad+C_{n-m} \mathbb{J}\left(\frac{1}{k^{m+1}} \frac{1}{k^{n-m}} k\left\|\left((k t)^{n+1} \tilde{f}(k t),\left(\frac{d}{d t}\right)^{n-m} \widetilde{\psi}(t)\right)\right\|_{\mathbb{B}}\right) .
\end{aligned}
$$


Отсюда получаем

$$
\begin{gathered}
\mathbb{J}\left(k\left\|\left(\tilde{f}(k t), t^{m+1} \widetilde{\psi}(t)\right)\right\|_{\mathbb{B}}\right) \leqslant c \sum_{j=0}^{n-m-1}\left\|\left(\tilde{f}(t), t^{j} t^{m+1} \widetilde{\psi}^{(j)}(t)\right)\right\|_{\mathbb{B}} \\
+c_{1} \mathbb{J}\left(k\left\|\left(\tilde{f}(k t), t^{n+1}\left(\frac{d}{d t}\right)^{n-m} \widetilde{\psi}(t)\right)\right\|_{\mathbb{B}}\right) .
\end{gathered}
$$

Учитьвая теперь, что $t^{n+1} \widetilde{\psi}^{(n-m)} \in \underset{n}{Z}$, и переходя к преобразованию Фурье, получаем (3.42). Чтобы получить оценку (3.43), снова воспользуемся теоремой 3.1. Согласно (3.5) для любого $a>0$ сушествует $a_{1}$ такое, что для любого $b$ найдутся $C_{1}$ и $N_{1}$ такие, что

$$
\begin{aligned}
\left\|\left(\tilde{f}(t), t^{j} t^{m+1} \widetilde{\psi}^{(j)}(t)\right)\right\|_{\mathbb{B}} \leqslant & C_{1}\left[\sup _{|x|<a_{1}} \hat{\mathbb{J}}\left(y^{b}\left\|L_{f}^{\omega}(x, y)\right\|_{\mathbb{B}}\right)+\sup _{|x|<a_{1}}\left\|L_{f}^{\omega_{0}}(x, 1)\right\|_{\mathbb{B}}\right] \\
& \times \mathscr{P}_{N_{1}}\left[F^{-1}\left[t^{j} t^{m+1} \widetilde{\psi}^{(j)}(t)\right](\xi)\right] \quad \forall \psi \in \mathscr{D}_{a},
\end{aligned}
$$

где $a_{1}, C_{1}$ и $N_{1}$ не зависят от $f$ и $\mathbb{B}$. Ясно, что для любого $a>0$ сушествуют $C_{2}$ и $N$ такие, что

$$
\mathscr{P}_{N_{1}}\left[F^{-1}\left[t^{j} t^{m+1} \widetilde{\psi}^{(j)}(t)\right](\xi)\right] \leqslant C_{2} \mathscr{P}_{N}[\varphi], \quad j=0,1, \ldots, n-m-1, \quad \varphi \in \mathscr{D}_{a} .
$$

Учитывая эту оценку, с помошью (3.45) оцениваем слагаемые справа в (3.44), стоящие под знаком суммы. Последнее же слагаемое в (3.44) оценивается с помощью уже полученного неравенства (3.39). В результате получаем оценку (3.43). Лемма доказана.

ЗАмЕчанИЕ 3.7 . Леммы 3.3 и 3.4 останутся справедливыми, если пространство $\mathscr{D}$ заменить на одно из пространств $E^{\uparrow}, E^{\downarrow}$ или $\mathscr{S}$, следует только оценки (3.37), (3.39) и (3.43) заменить на общие оценки класса (см. (2.12)-(2.14)).

ЛЕмма 3.5. Пусть $\mathbb{B}$ - банахово пространство, $\mathbb{J}(\varphi)$ - автомодельный функционал, $f(\xi) \in \mathbb{L}(\mathscr{D} \rightarrow \mathbb{B}) u \omega(\xi) \in \mathscr{D}$. Пусть, далее, существуют $A$ и $b$ такие, что

$$
\mathbb{J}\left(\left\|L_{f}^{\omega}\left(\frac{x}{k}, \frac{y}{k}\right)\right\|_{\mathbb{B}}\right) \leqslant \frac{A}{y^{b}}, \quad x^{2}+y^{2}=1, \quad y>0
$$

Тогда для любого а > 0 найдутся $C$ и d такие, что

$$
\mathbb{J}\left(\left\|L_{f}^{\omega}\left(\frac{x}{k}, \frac{y}{k}\right)\right\|_{\mathbb{B}}\right) \leqslant \frac{C}{y^{d}}, \quad|x|<a, \quad 0<y \leqslant 1 .
$$


ДоКАЗАТЕЛЬСТВО. Пусть $\alpha$ - Порядок $\mathbb{J}(\cdot), a>0$ и $|x|<a$. Положим

$$
x=k_{0} x_{0}, \quad y=k_{0} y_{0}, \quad \text { где } \quad x_{0}^{2}+y_{0}^{2}=1, \quad k_{0}^{2}=x^{2}+y^{2} .
$$

Рассмотрим два случая.

1) $k_{0} \leqslant 1$. Пользуясь аксиомой 5) автомодельных функционалов, имеем

$$
\begin{aligned}
\mathbb{J}\left(\left\|L_{f}^{\omega}\left(\frac{x}{k}, \frac{y}{k}\right)\right\|_{\mathbb{B}}\right) & =\mathbb{J}\left(\chi_{[1, \infty)}(k)\left\|L_{f}^{\omega}\left(\frac{x_{0}}{\frac{1}{k_{0}} k}, \frac{y_{0}}{\frac{1}{k_{0}} k}\right)\right\|_{\mathbb{B}}\right) \\
& =\mathbb{J}\left(\chi_{\left[\frac{1}{k_{0}}, \infty\right)}\left(\frac{1}{k_{0}} k\right)\left\|L_{f}^{\omega}\left(\frac{x_{0}}{\frac{1}{k_{0}} k}, \frac{y_{0}}{\frac{1}{k_{0}} k}\right)\right\|_{\mathbb{B}}\right) \\
& \leqslant \mathbb{J}\left(\chi_{[1, \infty)}\left(\frac{1}{k_{0}} k\right)\left\|L_{f}^{\omega}\left(\frac{x_{0}}{\frac{1}{k_{0}} k}, \frac{y_{0}}{\frac{1}{k_{0}} k}\right)\right\|_{\mathbb{B}}\right) \\
& \leqslant C_{\varepsilon}\left(\frac{1}{k_{0}}\right)^{\alpha+\varepsilon} \mathbb{J}\left(\left\|L_{f}^{\omega}\left(\frac{x_{0}}{k}, \frac{y_{0}}{k}\right)\right\|_{\mathbb{B}}\right) \leqslant C_{\varepsilon}\left(\frac{1}{k_{0}}\right)^{\alpha+\varepsilon} \frac{A}{y_{0}^{b}} \\
& \leqslant C_{\varepsilon} k_{0}^{b-\alpha-\varepsilon} \frac{A}{y^{d}} \leqslant C_{1} \frac{\left(x^{2}+y^{2}\right)^{\frac{b-\alpha-\varepsilon}{2}}}{y^{b}},
\end{aligned}
$$

откуда и следует (3.47) в этом случае.

2) $k_{0}>1$. Пользуясь аксиомой 5$)$, а также леммой 2.3 и оценкой класса (1.13), имеем

$$
\begin{aligned}
\mathbb{J}\left(\left\|L_{f}^{\omega}\left(\frac{x}{k}, \frac{y}{k}\right)\right\|_{\mathbb{B}}\right)= & \mathbb{J}\left(\chi_{\left[\frac{1}{k_{0}}, \infty\right)}\left(\frac{1}{k_{0}} k\right)\left\|L_{f}^{\omega}\left(\frac{x_{0}}{\frac{1}{k_{0}} k}, \frac{y_{0}}{\frac{1}{k_{0}} k}\right)\right\|_{\mathbb{B}}\right) \\
\leqslant & \mathbb{J}\left(\chi_{[1, \infty)}\left(\frac{1}{k_{0}} k\right)\left\|L_{f}^{\omega}\left(\frac{x_{0}}{\frac{1}{k_{0}} k}, \frac{y_{0}}{\frac{1}{k_{0}} k}\right)\right\|_{\mathbb{B}}\right) \\
& +\mathbb{J}\left(\chi_{\left[\frac{1}{k_{0}}, 1\right]}\left(\frac{1}{k_{0}} k\right)\left\|L_{f}^{\omega}\left(\frac{x_{0}}{\frac{1}{k_{0}} k}, \frac{y_{0}}{\frac{1}{k_{0}} k}\right)\right\|_{\mathbb{B}}\right) \\
\leqslant & c_{\varepsilon}\left(\frac{1}{k_{0}}\right)^{\alpha-\varepsilon} \mathbb{J}\left(\left\|L_{f}^{\omega}\left(\frac{x_{0}}{k}, \frac{y_{0}}{k}\right)\right\|_{\mathbb{B}}\right) \\
& +C_{\varepsilon} \operatorname{ess} \sup \frac{1}{1 \leqslant k \leqslant k_{0}}\left\|L_{f}^{\omega}\left(\frac{x}{k}, \frac{y}{k}\right)\right\|_{\mathbb{B}} \\
\leqslant & c_{\varepsilon}\left(\frac{1}{k_{0}}\right)^{\alpha-\varepsilon} \frac{A}{y_{0}^{b}}+C_{\varepsilon} k_{0}^{|\alpha|+\varepsilon} \frac{C_{1}}{y^{b_{1}}} k_{0}^{|b|} \leqslant \frac{C_{2}}{y^{d_{1}}} .
\end{aligned}
$$

Мы воспользовались тем, что $k_{0}^{2}=x^{2}+y^{2} \leqslant a^{2}+1$. Лемма доказана. 
ЗАмечаниЕ 3.8 . Лемма 3.5 остается справедливой, если $k$ заменить на $\frac{1}{k}$, т.е. если в условиях леммы вместо (3.46) выполнено соотношение

$$
\mathbb{J}\left(\left\|L_{f}^{\omega}(k x, k y)\right\|_{\mathbb{B}}\right) \leqslant \frac{A}{y^{b}} \text { при } x^{2}+y^{2}=1 .
$$

Тогда для любого $a>0$ найдутся $C$ и $d$ такие, что

$$
\mathbb{J}\left(\left\|L_{f}^{\omega}(k x, k y)\right\|_{\mathbb{B}}\right) \leqslant \frac{C}{y^{d}}, \quad|x|<a, \quad 0<y \leqslant 1
$$

ЗАмЕЧАниЕ 3.9. Если в условиях леммы 3.5 заменить $\mathscr{D}$ на одно из пространств $E^{\uparrow}, E^{\downarrow}$ или $\mathscr{S}$, то из (3.46) будет следовать общая оценка класса (см. (2.12)-(2.14)).

Например, если $f \in \mathbb{L}(\mathscr{S} \rightarrow \mathbb{B})$ и $\omega \in \mathscr{S}$ таковы, что выполнено условие (3.46), то сушествуют $a, b_{1}$ и $C_{1}$ такие, что

$$
\mathbb{J}\left(\left\|L_{f}^{\omega}\left(\frac{x}{k}, \frac{y}{k}\right)\right\|_{\mathbb{B}}\right) \leqslant \frac{C(1+|x|)^{a}}{y^{b_{1}}}, \quad x \in \mathbb{R}, \quad 0<y \leqslant 1
$$

Отметим, что если в (3.46) заменить $k$ на $\frac{1}{k}$, то $\left(3.47^{\prime}\right)$ будет иметь место, если в нем сделать такую же замену.

ТеОРема 3.2. Пусть $\mathbb{J}(\cdot)$ - автомодельный функиионал порядка $\alpha, \quad \mathbb{B}-$ банахово пространство, $f(\xi) \in \mathbb{L}(\mathscr{D} \rightarrow \mathbb{B})$ и функция $\omega(\xi) \in \mathscr{D}$ такова, ито $\int \omega(\xi) d \xi \neq 0$. Тогда следующие условия әквивалентны:

1) обобщенная функция $f(\xi)$ квазиасимптотически ограничена относительно J, m.e.

$$
\mathbb{J}\left(\left\|\left(f\left(\frac{\xi}{k}\right), \varphi(\xi)\right)\right\|_{\mathbb{B}}\right)<\infty \quad \forall \varphi \in \mathscr{D}
$$

2) существуют A и d такие, что

$$
\mathbb{J}\left(\left\|L_{f}^{\omega}\left(\frac{x}{k}, \frac{y}{k}\right)\right\|_{\mathbb{B}}\right) \leqslant \frac{A}{y^{d}}, \quad x^{2}+y^{2}=1, \quad y>0
$$

ДоКАЗАТЕЛЬСТвО вытекает из следствия 3.1 и лемм 3.3 и 3.5.

ЗАмЕчАниЕ 3.10 . Теорема 3.2 останется справедливой, если всюду заменить $k$ на $\frac{1}{k}$.

ЗАмечание 3.11 . Теорема 3.2 и замечание 3.10 останутся справедливыми, если $\mathscr{D}$ заменить на одно из пространств $E^{\uparrow}, E^{\downarrow}$ или $\mathscr{S}$. 
ТЕОРемА 3.3. Пусть $\mathbb{J}(\varphi)$ - автомодельный функционал порядка $\alpha, \mathbb{B}-$ банахово пространство, $f(\xi) \in \mathbb{L}(\mathscr{D} \rightarrow \mathbb{B})$. Тогда если обобщенная функиия $f(\xi)$ квазиасимптотически ограничена относительно $\mathbb{J}$ на $\stackrel{n}{\mathscr{D}}$, т.е.

$$
\mathbb{J}\left(\left\|\left(f\left(\frac{\xi}{k}\right), \varphi(\xi)\right)\right\|_{\mathbb{B}}\right)<\infty \quad \forall \varphi \in \stackrel{n}{\mathscr{D}},
$$

то для любого а > 0 существуют $A, N$ и $b$ такие, что

$$
\mathbb{J}\left(\left\|L_{f}^{\psi}\left(\frac{x}{k}, \frac{y}{k}\right)\right\|_{\mathbb{B}}\right) \leqslant \frac{A}{y^{b}} \mathscr{P}_{N}[\psi(\xi)], \quad x^{2}+y^{2}=1, \quad k>1, \quad \forall \psi \in \stackrel{n}{\mathscr{D}_{a}} .
$$

Пусть теперь для некоторого челого $n$ нашлась функиия $\omega(\xi) \in \stackrel{n}{\mathscr{D}} \backslash \stackrel{n+1}{\mathscr{D}}$ такая, что существуют $A$ и d такие, что

$$
\mathbb{J}\left(\left\|L_{f}^{\omega}\left(\frac{x}{k}, \frac{y}{k}\right)\right\|_{\mathbb{B}}\right) \leqslant \frac{A}{y^{d}}, \quad x^{2}+y^{2}=1, \quad y>0 .
$$

Тогда обобщенная функщия $f(\xi)$ квазиасимптотически ограничена относительно $\mathbb{J}$ на $\stackrel{m}{\mathscr{D}}$, m.e.

$$
\mathbb{J}\left(\left\|\left(f\left(\frac{\xi}{k}\right), \varphi(\xi)\right)\right\|_{\mathbb{B}}\right)<\infty \quad \forall \varphi \in \stackrel{m}{\mathscr{D}},
$$

где $m=\min \{n,[-\alpha]\}$.

ДоКАЗАТЕЛЬСТвО вытекает из следствия 3.1 и лемм 3.4 и 3.5 .

ЗАмечАниЕ 3.12 . Теорема 3.3 останется справедливой, если $\mathscr{D}$ заменить на одно из пространств $E^{\uparrow}, E^{\downarrow}$ или $\mathscr{S}$.

ТЕОРемА 3.4. Пусть $\rho(k)$ - автомодельная функция порядка $\alpha, f(\xi) \in$

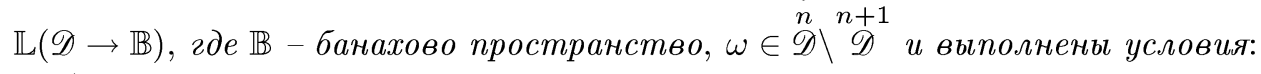

1) в $\mathbb{B}$ существует

$$
\lim _{k \rightarrow+\infty} \frac{1}{\rho(k)} L_{f}^{\omega}\left(\frac{x}{k}, \frac{y}{k}\right)=c(x, y), \quad(x, y) \in \mathbb{R} \times(0,1] ;
$$

2) существуют $A$ и $b$ такие, что

$$
\sup _{k \geqslant 1} \frac{1}{\rho(k)}\left\|L_{f}^{\omega}\left(\frac{x}{k}, \frac{y}{k}\right)\right\|_{\mathbb{B}} \leqslant \frac{A}{y^{b}}, \quad x^{2}+y^{2}=1, \quad y>0 .
$$

Тогда функиия $f(\xi)$ обладает квазиасимптотикой в нуле на $\stackrel{n}{D}$ относительно $\rho(k)$, т.е. в $\mathbb{B}$ существует

$$
\lim _{k \rightarrow+\infty} \frac{1}{\rho(k)}\left(f\left(\frac{\xi}{k}\right), \varphi(\xi)\right)=c_{\varphi} \quad \forall \varphi \in \stackrel{n}{\mathscr{D}} .
$$

Здесь $c_{\varphi} \in \mathbb{B}$. 
ДоКАЗАТЕЛЬСТво. Введем банахово пространство

$$
H=\left\{\eta(k) \in C([1,+\infty) \rightarrow \mathbb{B}): \quad \exists \lim _{k \rightarrow \infty} \frac{1}{\rho(k)} \eta(k) \text { в } \mathbb{B}\right\}
$$

с нормой $\|\eta\|_{H}=\sup _{k \geqslant 1} \frac{1}{\rho(k)}\|\eta(k)\|_{\mathbb{B}}$ и локально выпуклое пространство

$$
\mathscr{L}=\left\{\eta(k) \in C([1,+\infty) \rightarrow \mathbb{B}): \quad Q_{N}[\eta]=\sup _{1 \leqslant k \leqslant N} \frac{1}{\rho(k)}\|\eta(k)\|_{\mathbb{B}}<\infty\right\}
$$

со счетной системой полунорм $Q_{N}[\cdot], \quad N=2,3, \ldots$ Ясно, что $H \subset \mathscr{L}$. Зададим функцию $g(\xi) \in \mathbb{L}(\mathscr{D} \rightarrow \mathscr{L})$ формулой

$$
(g(\xi), \varphi(\xi))=\left(f\left(\frac{\xi}{k}\right), \varphi(\xi)\right)=k(f(\xi), \varphi(k \xi)) .
$$

Отметим, что в силу (3.51) функция

$$
L_{g}^{\omega}(x, y)=\left(f(\xi), \frac{k}{y} \omega\left(\frac{x-k \xi}{y}\right)\right)=L_{f}^{\omega}\left(\frac{x}{k}, \frac{y}{k}\right)
$$

прикаждом $(x, y) \in \mathbb{R} \times(0,1]$ принадлежит $H$. Согласно лемме П.6 она измерима по $(x, y)$ как функция со значениями в $H$.

Теперь для $L_{g}^{\omega}(x, y)$ по лемме 3.5 из (3.52) следует условие (3.2) теоремы 3.1 при $\mathbb{J}(\cdot)=\mathbb{J}^{\infty}\left(\frac{1}{\rho(k)} \cdot\right)$, а потому $(g(\xi), \varphi(\xi))=\left(f\left(\frac{\xi}{k}\right), \varphi(\xi)\right) \in H$ для любой $\varphi \in \stackrel{n}{\mathscr{D}}$, что и доказывает (3.53). Теорема доказана.

ЗАмечаниЕ 3.13 . Если $\int \omega(\xi) d \xi \neq 0$, то (3.53) выполняется для всех $\varphi \in \mathscr{D}$.

ЗАмЕчАниЕ 3.14 . Теорема 3.4 останется справедливой, если всюду в $L_{f}^{\omega}\left(\frac{x}{k}, \frac{y}{k}\right)$ и $f\left(\frac{\xi}{k}\right)$ заменить $k$ на $\frac{1}{k}$.

ЗАмечАниЕ 3.15 . Теорема 3.4 и замечания $3.13,3.14$ останутся справедливыми, если пространство $\mathscr{D}$ заменить на одно из пространств $E^{\uparrow}, E^{\downarrow}$ или $\mathscr{S}$.

ТЕОРема 3.5. Пусть $\rho(k)$ - автомодельная функиия порядка $\alpha, f(\xi) \in$ $\mathbb{L}(\mathscr{D} \rightarrow \mathbb{B})$, цде $\mathbb{B}$ - банахово пространство, $\omega \in \stackrel{n}{\mathscr{D} \backslash} \backslash \stackrel{n+1}{\mathscr{D}}$ и выполнены условия:

1) в $\mathbb{B}$ существует

$$
\lim _{k \rightarrow+\infty} \frac{1}{\rho(k)}\left(f(\xi+k), \omega\left(\frac{\xi}{y}\right)\right)=c(y), \quad y \in(0,1] ;
$$

2) существуют $A$ и $b$ такие, что

$$
\sup _{k \geqslant 1} \frac{1}{\rho(k)}\left\|\left(f(\xi+k), \omega\left(\frac{\xi}{y}\right)\right)\right\|_{\mathbb{B}} \leqslant \frac{A}{y^{b}}, \quad y \in(0,1) .
$$

Тогда функиия $f(\xi)$ обладает асимптотикой по сдвигам на $\stackrel{n}{D}$ относительно $\rho(k)$, т.е. в $\mathbb{B}$ существует

$$
\lim _{k \rightarrow+\infty} \frac{1}{\rho(k)}(f(\xi+k), \varphi(\xi))=c_{\varphi} \quad \forall \varphi \in \stackrel{n}{\mathscr{D}} .
$$

Здесь $c_{\varphi} \in \mathbb{B}$. 
ДоКАЗАТЕЛЬСТво. Как и при доказательстве теоремы 3.4 , вводим пространства $H$ и $\mathscr{L}$ по формулам (3.54) и (3.55). Зададим функцию $g(\xi) \in \mathbb{L}(\mathscr{D} \rightarrow \mathbb{B})$ формулой

$$
(g(\xi), \varphi(\xi))=(f(\xi+k), \varphi(\xi))=(f(\xi), \varphi(\xi-k)) \quad \forall \varphi \in \mathscr{D} .
$$

Заметим, что функция $L_{g}^{\omega}(x, y)=\left(f(\xi+k), \frac{1}{y} \omega\left(\frac{\xi-x}{y}\right)\right)=L_{f}^{\omega}(x+k, y)$ при каждом $(x, y) \in \mathbb{R} \times(0,+\infty)$ принадлежит $H$. Непрерывность по $k$ очевидна и

$$
\begin{aligned}
\lim _{k \rightarrow+\infty} \frac{1}{\rho(k)} L_{f}^{\omega}(x+k, y) & =\lim _{k \rightarrow+\infty} \frac{1}{\rho(k-x)} L_{f}^{\omega}(k, y) \\
& =\lim _{k \rightarrow+\infty} \frac{\rho(k)}{\rho(k-x)} \frac{1}{\rho(k)}\left(f(\xi), \frac{1}{y} \omega\left(\frac{\xi-k}{y}\right)\right) \\
& =\frac{1}{y} \lim _{k \rightarrow+\infty} \frac{\rho(k)}{\rho(k-x)} \frac{1}{\rho(k)}\left(f(\xi+k), \omega\left(\frac{\xi}{y}\right)\right) \\
& =\frac{1}{y} c(y) \lim _{k \rightarrow+\infty} \frac{\rho(k)}{\rho(k-x)}=\frac{1}{y} c(y) .
\end{aligned}
$$

Здесь мы воспользовались условием (3.56).

Покажем, что $L_{g}^{\omega}(x, y)$ удовлетворяет условию (3.2) для банахова пространства $H$ и автомодельного функционала $\mathbb{J}(\cdot)=\mathbb{J}^{\infty}\left(\frac{1}{\rho(k)} \cdot\right)$. Пусть $a>0$ и $|x|<a$. При $k_{0}>a+1$ имеем

$$
\sup _{k \geqslant 1} \frac{1}{\rho(k)}\left\|L_{f}^{\omega}(x+k, y)\right\|_{\mathbb{B}} \leqslant\left\{\sup _{1 \leqslant k \leqslant k_{0}-x}+\sup _{k>k_{0}-x}\right\} \frac{1}{\rho(k)}\left\|L_{f}^{\omega}(x+k, y)\right\|_{\mathbb{B}} .
$$

Первое слагаемое справа в (3.59) в силу общей оценки класса $(|x|<a, 1 \leqslant k \leqslant$ $\left.k_{0}+a\right)$ не превосходит $\frac{A_{2}}{y^{b_{2}}}$ при некоторых $A_{2}$ и $b_{2}$. Оценим второе слагаемое. Имеем

$$
\sup _{k>k_{0}-x} \frac{1}{\rho(k)}\left\|L_{f}^{\omega}(x+k, y)\right\|_{\mathbb{B}}=\sup _{k>k_{0}-x} \frac{\rho(k+x)}{\rho(k)} \frac{1}{\rho(k+x)}\left\|L_{f}^{\omega}(x+k, y)\right\|_{\mathbb{B}} \leqslant \frac{A_{1}}{y^{b_{1}}},
$$

так как $\frac{\rho(k+x)}{\rho(k)}$ ограничено $(|x|<a)$, а

$$
\sup _{k>k_{0}-x} \frac{1}{\rho(k+x)}\left\|L_{f}^{\omega}(x+k, y)\right\|_{\mathbb{B}}=\sup _{k>k_{0}} \frac{1}{\rho(k)}\left\|L_{f}^{\omega}(k, y)\right\|_{\mathbb{B}} \leqslant \frac{A}{y^{b+1}}
$$

по условию (3.57). Итак, условие (3.2) выполнено.

Измеримость $L_{g}^{\omega}(x, y)$ по $(x, y) \in \mathbb{R} \times(0,1)$ как функции со значениями в $H$ следует из леммы П.6. Из изложенного ранее по теореме 3.1 получаем (3.58). Теорема доказана.

ЗАмечание 3.16. Если $\int \omega(\xi) d \xi \neq 0$, то (3.60) выполняется для всех $\varphi \in \mathscr{D}$.

ЗАмЕчАниЕ 3.17 . В условиях теоремы 3.5 обязательно $c(y)=y c$, где $c \in \mathbb{B}$, а $c_{\varphi}=c \int \varphi(\xi) d \xi$.

ЗАмечание 3.18 . Теорема 3.5 и замечания $3.16,3.17$ останутся справедливыми, если пространство $\mathscr{D}$ заменить на одно из пространств $E^{\uparrow}, E^{\downarrow}$ или $\mathscr{S}$. 


\section{§ 4. Тауберова теорема для банахова пространства, имеющего представление группы сдвигов}

Пусть $\mathbb{B}$ - банахово пространство и в нем задано представление группы сдвигов $\left\{T_{a} \in \mathbb{L}(\mathbb{B} \rightarrow \mathbb{B}), a \in \mathbb{R}\right\}$ класса $\left(C_{0}\right)$. Это означает, что каждому $a \in \mathbb{R}$ сопоставлен непрерывный оператор $T_{a}: \mathbb{B} \rightarrow \mathbb{B}$ и выполнены свойства:

1) $T_{a} T_{b}=T_{a+b}$ для всех $a, b \in \mathbb{R}$;

2) $\lim _{\Delta \rightarrow 0}\left\|T_{\Delta} e-e\right\|_{\mathbb{B}}=0$ для любого $e \in \mathbb{B}$.

Известно [10], что тогда сушествуют постоянные $M$ и $\beta$ такие, что

$$
\left\|T_{a}\right\| \leqslant C(a)=M e^{\beta|a|}, \quad a \in \mathbb{R} .
$$

ТЕОРемА 4.1. Пусть $\mathbb{J}$ - автомодельный функиионал порядка $\alpha, \mathbb{B}-$ банахово и $\mathscr{L}$ - локально выпуклое пространства, причем $\mathbb{B} \subset \mathscr{L}$ линейно и непрерывно. Кроме того, в $\mathscr{L}$ действует представление $\left\{T_{a} \in \mathbb{L}(\mathscr{L} \rightarrow \mathscr{L}), a \in \mathbb{R}\right\}$ группь сдвигов и оно индуцирует представление $\left\{T_{a} \in \mathbb{L}(\mathbb{B} \rightarrow \mathbb{B}), a \in \mathbb{R}\right\}$ группь сдвигов класса $\left(C_{0}\right)$ на $\mathbb{B}$. Пусть, далее, функиия $f \in \mathbb{L}(\mathscr{D} \rightarrow \mathscr{L})$ такая, что

$$
(f(\xi), \varphi(\xi-a))=T_{a}(f(\xi), \varphi(\xi)), \quad a \in \mathbb{R}, \quad \forall \varphi \in \mathscr{D} .
$$

Предположим, что для некоторой $\omega(\xi) \in \stackrel{n}{\mathscr{D}} \backslash \stackrel{n+1}{\mathscr{D}}$ функция $L_{f}^{\omega}(0, y)$ измерима по $y \in(0,1]$ как функция со значениями в $\mathbb{B}$, причем

$$
\hat{\mathbb{J}}\left(\left\|L_{f}^{\omega}(0, y)\right\|_{\mathbb{B}}\right)<\infty
$$

Если $n \geqslant 0$, то пусть найдется еще функция $\omega_{0} \in \mathscr{D}, \quad \int \omega_{0}(\xi) d \xi \neq 0$, такая, что $L_{f}^{\omega_{0}}(0,1) \in \mathbb{B}$. Тогда

$$
f \in \mathbb{L}(\mathscr{D} \rightarrow \mathbb{B}) \quad u \quad \mathbb{J}\left(\left\|\left(f\left(\frac{\xi}{k}\right), \varphi(\xi)\right)\right\|_{\mathbb{B}}\right)<\infty \quad \forall \varphi \in \stackrel{m}{\mathscr{D}}
$$

где $m=\min \{n,[-\alpha]\}$. Более того, для любого а> 0 существуют $C$ и $N$ такие, что

$$
\mathbb{J}\left(\left\|\left(f\left(\frac{\xi}{k}\right), \varphi(\xi)\right)\right\|_{\mathbb{B}}\right) \leqslant C \hat{\mathbb{J}}\left(\left\|L_{f}^{\omega}(0, y)\right\|_{\mathbb{B}}\right) \mathscr{P}_{N}[\varphi] \quad \forall \varphi \in \stackrel{n}{\mathscr{D}}_{a}
$$

Кроме того, для любого а> 0 существуют $C$ и $N$ такие, что

$$
\begin{aligned}
& \mathbb{J}\left(\left\|\left(f\left(\frac{\xi}{k}\right), \varphi(\xi)\right)\right\|_{\mathbb{B}}\right) \leqslant \\
& \quad \leqslant C\left[\hat{\mathbb{J}}\left(\left\|L_{f}^{\omega}(0, y)\right\|_{\mathbb{B}}\right)+\left\|L_{f}^{\omega_{0}}(0,1)\right\|_{\mathbb{B}}\right] \mathscr{P}_{N}[\varphi] \quad \forall \varphi \in \stackrel{m}{\mathscr{D}}_{a} .
\end{aligned}
$$

Здесь, как и в предьдущих теоремах, $C$ и $N$ не зависят от $f, \mathbb{B} и \varphi$. 
ДокАЗАТЕЛЬСТво. Прежде всего заметим, что

$$
\begin{aligned}
L_{f}^{\omega}(x+a, y) & =\left(f(\xi), \frac{1}{y} \omega\left(\frac{x-(\xi-a)}{y}\right)\right)=T_{a}\left(f(\xi), \frac{1}{y} \omega\left(\frac{x-\xi}{y}\right)\right) \\
& =T_{a} L_{f}^{\omega}(x, y) .
\end{aligned}
$$

Поэтому п.в. $L_{f}^{\omega}(x, y)=T_{x} L_{f}^{\omega}(0, y) \in \mathbb{B}$. Покажем, что $L_{f}^{\omega}(x, y)$ измерима по $(x, y)$ как функция со значениями в $\mathbb{B}$. Действительно, в силу измеримости $L_{f}^{\omega}(0, y)$ сушествуют непрерывные по $y$ функции $G_{n}(y)$ со значениями в $\mathbb{B}$ такие, что

$$
\left\|L_{f}^{\omega}(0, y)-G_{n}(y)\right\|_{\mathbb{B}} \underset{n \rightarrow \infty}{\longrightarrow} 0 \text { п.в. по } y \in(0,1) .
$$

Заметим, что функции $T_{x} G_{n}(y)$ непрерывны по $(x, y)$ как функции со значениями в $\mathbb{B}$, так как

$$
\begin{aligned}
\left\|T_{x} G_{n}(y)-T_{x_{1}} G_{n}\left(y_{1}\right)\right\|_{\mathbb{B}} \leqslant & \left\|T_{x} G_{n}(y)-T_{x_{1}} G_{n}(y)\right\|_{\mathbb{B}}+\left\|T_{x_{1}} G_{n}(y)-T_{x_{1}} G_{n}\left(y_{1}\right)\right\|_{\mathbb{B}} \\
\leqslant & \left\|T_{x}\right\|\left\|T_{x_{1}-x} G_{n}(y)-G_{n}(y)\right\|_{\mathbb{B}} \\
& +\left\|T_{x_{1}}\right\|\left\|G_{n}(y)-G_{n}\left(y_{1}\right)\right\|_{\mathbb{B}} \underset{x_{1} \rightarrow x, y_{1} \rightarrow y}{\longrightarrow} 0 .
\end{aligned}
$$

Первое из слагаемых в этом неравенстве стремится к нулю в силу свойства 2) группы класса $\left(C_{0}\right)$, а второе - в силу непрерывности $G_{n}(y)$. Так как

$$
\left\|L_{f}^{\omega}(x, y)-T_{x} G_{n}(y)\right\|_{\mathbb{B}} \leqslant\left\|T_{x}\right\|\left\|L_{f}^{\omega}(0, y)-G_{n}(y)\right\|_{\mathbb{B}} \underset{n \rightarrow \infty}{\longrightarrow} 0 \text { п.в. по } y \in(0,1),
$$

то $L_{f}^{\omega}(x, y)$ есть предел п.в. непрерывных функций со значениями в $\mathbb{B}$, т.е. является измеримой функцией.

Пусть $d>0$; тогда при $|x|<d$ имеем

$$
\begin{aligned}
\mathbb{J}\left(\left\|L_{f}^{\omega}\left(x, \frac{1}{k}\right)\right\|_{\mathbb{B}}\right) & =\mathbb{J}\left(\left\|T_{x} L_{f}^{\omega}\left(0, \frac{1}{k}\right)\right\|_{\mathbb{B}}\right) \leqslant\left\|T_{x}\right\| \mathbb{J}\left(\left\|L_{f}^{\omega}\left(0, \frac{1}{k}\right)\right\|_{\mathbb{B}}\right) \\
& \leqslant \mathrm{C}(d) \mathbb{J}\left(\left\|L_{f}^{\omega}\left(0, \frac{1}{k}\right)\right\|_{\mathbb{B}}\right)=C(d) \hat{\mathbb{J}}\left(\left\|L_{f}^{\omega}(0, y)\right\|_{\mathbb{B}}\right),
\end{aligned}
$$

т.е. вьполнено условие (3.2) теоремы 3.1 при $b=0$.

Если $n \geqslant 0$, то функция $L_{f}^{\omega_{0}}(x, 1)=T_{x} L_{f}^{\omega_{0}}(0,1)$ непрерывна по $x$ со значениями в $\mathbb{B}$. Поэтому для любого $d>0$

$$
\sup _{|x|<d}\left\|L_{f}^{\omega_{0}}(x, 1)\right\|_{\mathbb{B}} \leqslant \sup _{|x|<d}\left\|T_{x}\right\|\left\|L_{f}^{\omega_{0}}(0,1)\right\|_{\mathbb{B}} \leqslant C(d)\left\|L_{f}^{\omega_{0}}(0,1)\right\|_{\mathbb{B}}
$$

Значит, выполнены все условия теоремы 3.1. Следовательно, $f \in \mathbb{L}(\mathscr{D} \rightarrow \mathbb{B})$. 
Далее, при $|x|<a_{1}, 0<y \leqslant 1$ имеем

$$
\begin{aligned}
\mathbb{J}\left(\left\|L_{f}^{\omega}\left(\frac{x}{k}, \frac{y}{k}\right)\right\|_{\mathbb{B}}\right) & =\mathbb{J}\left(\left\|T_{\frac{x}{k}} L_{f}^{\omega}\left(0, \frac{y}{k}\right)\right\|_{\mathbb{B}}\right) \leqslant \mathbb{J}\left(\left\|T_{\frac{x}{k}}\right\|\left\|_{f}^{\omega}\left(0, \frac{y}{k}\right)\right\|_{\mathbb{B}}\right) \\
& \leqslant C\left(a_{1}\right) \mathbb{J}\left(\left\|L_{f}^{\omega}\left(0, \frac{y}{k}\right)\right\|_{\mathbb{B}}\right) \\
& \leqslant C_{\varepsilon} C\left(a_{1}\right)\left(\frac{1}{y}\right)^{\alpha+\varepsilon} \mathbb{J}\left(\left\|L_{f}^{\omega}\left(0, \frac{1}{k}\right)\right\|_{\mathbb{B}}\right) .
\end{aligned}
$$

Здесь мы воспользовались соотношением (2.4) для автомодельных функционалов, где $\varkappa=\frac{1}{y} \geqslant 1$.

Неравенства (4.4) и (4.5) теперь непосредственно следуют из леммы 3.4 (в которой $b$ следует считать бо́льшим $|\alpha|+\varepsilon)$. Теорема доказана.

ЗАмечАниЕ 4.1. В условиях теоремы 4.1 для любого $a>0$ найдутся $C_{2}$ и $N$ такие, что

$$
\begin{aligned}
& \mathbb{J}\left(\left\|\left(f\left(\frac{\xi}{k}\right), \varphi(\xi)\right)\right\|_{\mathbb{B}}\right)+\left\|L_{f}^{\varphi_{0}}(0,1)\right\|_{\mathbb{B}} \leqslant C_{2}\left[\mathbb{J}\left(\left\|\left(f\left(\frac{\xi}{k}\right), \omega(\xi)\right)\right\|_{\mathbb{B}}\right)\right. \\
& \left.\quad+\left\|L_{f}^{\omega_{0}}(0,1)\right\|_{\mathbb{B}}\right]\left[\mathscr{P}_{N}[\varphi]+\mathscr{P}_{N}\left[\varphi_{0}\right]\right] \quad \forall \varphi \in \mathscr{m}_{a}, \quad \varphi_{0} \in \mathscr{D}_{a} .
\end{aligned}
$$

Здесь $C_{2}$ и $N$ не зависят от $f, \mathbb{B}, \varphi$ и $\varphi_{0}$.

Действительно, сперва заметим, что $\left(f\left(\frac{\xi}{k}\right), \psi(\xi)\right)=L_{f}^{\psi}\left(0, \frac{1}{k}\right)$ и $L_{f}^{\varphi_{0}}(0,1)=$ $\left(f(\xi), \varphi_{0}(\xi)\right)$. Из формулы $(3.5)$, учитывая (4.7) и (4.6), получаем, что для любого $a>0$ сушествуют $C$ и $N$ такие, что

$$
\left\|L_{f}^{\varphi_{0}}(0,1)\right\|_{\mathbb{B}} \leqslant C\left[\hat{\mathbb{J}}\left(\left\|L_{f}^{\omega}(0, y)\right\|_{\mathbb{B}}\right)+\left\|L_{f}^{\omega_{0}}(0,1)\right\|_{\mathbb{B}}\right] \mathscr{P}_{N}\left[\varphi_{0}\right] \quad \forall \varphi_{0} \in \mathscr{D}_{a} .
$$

Комбинируя эту оценку с (4.5), получим оценку (4.9).

\section{§ 5. Асимптотические свойства решений задачи Коши для уравнения теплопроводности}

В этом параграфе полученные выше теоремы применяются для изучения асимптотических свойств решений задачи Коши для уравнения теплопроводности

$$
\frac{\partial}{\partial t} u(x, t)=\frac{\partial^{2}}{\partial x^{2}} u(x, t), \quad \lim _{t \rightarrow+0} u(x, t)=f(x) \quad\left(\text { в } \mathscr{S}^{\prime}\right), \quad f \in \mathscr{S}^{\prime}, \quad x \in \mathbb{R}, \quad t>0,
$$

в классе не более чем полиномиально растуших функций, т.е. таких, что $|u(x, t)|<$ $C \frac{1}{t^{b}}\left(|x|^{N}+|t|^{N}+1\right)$ для некоторых $b, C$ и $N$, зависяших от $u$. Как известно, в этом классе сушествует единственное решение задачи (5.1), задаваемое формулой

$$
u(x, t)=\left(f(\xi), \frac{1}{2 \sqrt{\pi t}} e^{-\frac{(x-\xi)^{2}}{4 t}}\right) \equiv L_{f}^{\omega}(x, y)
$$


где $\omega(\xi)=\frac{1}{\sqrt{\pi}} e^{-\xi^{2}}$ и $y=2 \sqrt{t}$.

ТЕОРема 5.1. Пусть $\rho(k)$ - автомодельная функиия. Для того чтобь начальная функиия $f \in \mathscr{S}^{\prime}$ задачи (5.1) обладала квазиасимптотикой на бесконечности относительно $\rho(k)$, т.е.

$$
\frac{1}{\rho(k)}(f(k \xi), \varphi(\xi)) \underset{k \rightarrow+\infty}{\longrightarrow} c_{\varphi} \quad \forall \varphi \in \mathscr{S},
$$

необходимо и достаточно, чтобь решение задачи (5.1) удовлетворяло следующим условиям:

1) существует предел

$$
\frac{1}{\rho(k)} u\left(k x, k^{2} t\right) \underset{k \rightarrow+\infty}{\longrightarrow} u_{0}(x, t), \quad x \in \mathbb{R}, \quad t>0
$$

2) существуют $A$ u $d$ такие, что

$$
\left|\frac{1}{\rho(k)} u\left(k x, k^{2} t\right)\right| \leqslant \frac{A}{t^{d}}, \quad x^{2}+t^{2}=1 .
$$

ДокАЗАТЕЛЬСтво. Необходимость. Пусть выполнено (5.3). Подставляя в это соотношение вместо $\varphi$ функцию $\frac{1}{y} \omega\left(\frac{x-\xi}{y}\right)$, где $y=2 \sqrt{t}$ и $\omega(\xi)=\frac{1}{\sqrt{\pi}} e^{-\xi^{2}}$, имеем

$$
\begin{aligned}
\lim _{k \rightarrow+\infty} \frac{1}{\rho(k)}\left(f(k \xi), \frac{1}{2 \sqrt{\pi t}} e^{-\frac{(x-\xi)^{2}}{4 t}}\right) & =\lim _{k \rightarrow+\infty} \frac{1}{\rho(k)}\left(f(\xi), \frac{1}{2 \sqrt{\pi k^{2} t}} e^{-\frac{(k x-\xi)^{2}}{4 t k^{2}}}\right) \\
& =\lim _{k \rightarrow+\infty} \frac{1}{\rho(k)} u\left(k x, k^{2} t\right) \underset{k \rightarrow+\infty}{\longrightarrow} c_{\omega}(x, t)
\end{aligned}
$$

что и доказывает (5.4). Оценка (5.5) легко следует из общей оценки класса для основной функции $\frac{1}{2 \sqrt{t}} \omega\left(\frac{x-\xi}{2 \sqrt{t}}\right)($ см. $(2.14))$.

Достаточность. Пусть выполнены условия (5.4) и (5.5). Согласно теореме 3.4 (см. также замечания 3.13 и 3.14 ) из этих условий вытекает (5.3) для любой функции $\varphi \in \stackrel{n}{\mathscr{S}}$. Так как $\int \omega(\xi) d \xi=1$, то $\stackrel{n}{\mathscr{S}}=\mathscr{S}$. Теорема доказана.

ТЕОРема 5.2. Пусть $\rho(k)$ - автомодельная функиия. Для того чтобы начальная функиия $f \in \mathscr{S}^{\prime}$ задачи (5.1) обладала асимптотикой по сдвигам на $+\infty$ относительно $\rho(k)$, т.е.

$$
\frac{1}{\rho(k)}(f(\xi+k), \varphi(\xi)) \underset{k \rightarrow+\infty}{\longrightarrow} c_{\varphi} \quad \forall \varphi \in \mathscr{S},
$$

необходимо и достаточно, чтобь решение задачи (5.1) удовлетворяло следующим условиям:

1) существует предел

$$
\frac{1}{\rho(x)} u(x, t) \underset{x \rightarrow+\infty}{\longrightarrow} c(t), \quad t>0
$$

2) найдутся $A$ и d такие, что

$$
\left|\frac{1}{\rho(x)} u(x, t)\right| \leqslant \frac{A}{t^{d}}, \quad x>1, \quad 0<t<1 .
$$


ДокАЗАТЕЛЬСТво аналогично доказательству предыдущей теоремы, только вместо теоремы 3.4 следует пользоваться теоремой 3.5 и замечаниями $3.16-3.18$.

ЗАмЕЧАНИЕ 5.1. Нетрудно видеть, что в (5.6) $c_{\varphi}=c \int \varphi(\xi) d \xi$ и, следовательно, в $(5.7) c(t)=c$, т.е. не зависит от $t$.

\section{§ 6. Банаховы пространства обобщенных функций}

Пусть $\mathbb{B}$ - банахово пространство, для которого выполнены следующие условия (аксиомы).

I. $\mathscr{D} \subset \mathbb{B} \subset \mathscr{D}^{\prime}$, причем $\mathscr{D}$ плотно вложено в $\mathbb{B}$.

II. $\mathbb{B}$ инвариантно относительно группы сдвигов, действующих в $\mathscr{D}^{\prime}$, т.е. каждому $a \in \mathbb{R}$ сопоставляется линейный оператор $T_{a}: \mathbb{B} \mapsto \mathbb{B}$, причем предполагается, что этот оператор ограничен и

$$
\left\|T_{\xi}\right\| \leqslant C(a) \text { для }|\xi| \leqslant a
$$

где $C(a)$ - некоторая локально ограниченная функция.

Из плотности и непрерывности вложения $\mathscr{D}$ в $\mathbb{B}$ вытекает, что

$$
\lim _{\Delta \rightarrow 0}\left\|T_{\Delta} e-e\right\|_{\mathbb{B}}=0 \quad \forall e \in \mathbb{B}
$$

таким образом, группа $\left\{T_{a}, a \in \mathbb{R}\right\}$ является группой класса $\left(C_{0}\right)$, в частности,

$$
\left\|T_{a}\right\| \leqslant M(1+|a|)^{Q} e^{\beta|a|}, \quad a \in \mathbb{R},
$$

для некоторых $Q, M$ и $\beta$ (см. [10]). Ясно, что

$$
\mathscr{D} \subset \mathbb{B}^{*} \subset \mathscr{D}^{\prime}
$$

причем второе вложение плотно (что, вообще говоря, нельзя сказать о первом вложении).

Покажем, что $E_{b}$ при любом $b \geqslant \beta$ (см. соотношение $\left.(6.2)\right)$ плотно и непрерывно вложено в $\mathbb{B}$, т.е.

$$
\mathscr{D} \subset E_{b} \subset \mathbb{B}
$$

Для этого достаточно доказать, что $E_{\beta} \subset \mathbb{B}$.

Пусть $\varphi(\eta) \in E_{\beta}$. Возьмем специальное разбиение еденицы:

$$
\psi(\eta) \in \mathscr{D}, \quad \operatorname{supp} \psi \subset[-1,+1], \quad 1=\sum_{n=-\infty}^{\infty} \psi(\eta-n)
$$

Тогда $\varphi(\eta)=\sum_{n=-\infty}^{+\infty} \psi(\eta-n) \varphi(\eta)$. Оценим отдельные слагаемые этой суммы. Согласно (6.2) имеем

$$
\begin{aligned}
\|\varphi(\eta) \psi(\eta-n)\|_{\mathbb{B}} & \leqslant M(1+|n|)^{Q} e^{\beta|n|}\|\varphi(\eta+n) \psi(\eta)\|_{\mathbb{B}} \\
& =\frac{M}{(1+|n|)^{2}}\left\|(1+|n|)^{2+Q} e^{\beta|n|} \varphi(\eta+n) \psi(\eta)\right\|_{\mathbb{B}}
\end{aligned}
$$


Заметим, что множество функций $\left\{g_{n}(\eta)=(1+|n|)^{2+Q} e^{\beta|n|} \varphi(\eta+n) \psi(\eta) ;-\infty<\right.$ $n<+\infty\}$ ограничено в $\mathscr{D}$, так как все они принадлежат $\mathscr{D}_{[-1,+1]}$ и вьполнена оценка

$$
\begin{aligned}
\mathscr{P}_{N}\left[g_{n}(\eta)\right] & =\max _{j \leqslant N} \sup _{\eta}(1+|\eta|)^{N}(1+|n|)^{2+Q} e^{\beta|n|}\left|(\varphi(\eta+n) \psi(\eta))^{(j)}\right| \\
& \leqslant C_{1} \max _{j \leqslant N} \sup _{|\eta|<1}(1+|n|)^{2+Q} e^{\beta|n|}\left|\varphi^{(j)}(\eta+n)\right| \\
& =C_{2} \max _{j \leqslant N} \sup _{|\xi-n|<1}(1+|n|)^{2+Q} e^{\beta|n|}\left|\varphi^{(j)}(\xi)\right| \\
& \leqslant C_{3} \max _{j \leqslant N} \sup _{\xi}(1+|\xi|+1)^{2+Q} e^{\beta(|\xi|+1)}\left|\varphi^{(j)}(\xi)\right| \leqslant C_{4} P_{\beta, N+Q+2}[\varphi]
\end{aligned}
$$

для $N=0,1, \ldots$ В силу непрерывности вложения $\mathscr{D}$ в $\mathbb{B}$ существуют $C$ и $N_{0}$ такие, что $\|h\|_{\mathbb{B}} \leqslant C \mathscr{P}_{N_{0}}[h]$ для любого $h \in \mathscr{D}_{[-1,+1]}$. Поэтому

$$
\left\|g_{n}(\eta)\right\|_{\mathbb{B}} \leqslant C \mathscr{P}_{N_{0}}\left[g_{n}(\eta)\right] \leqslant C_{5} P_{\beta, N_{0}+Q+2}[\varphi] .
$$

Отсюда

$$
\begin{aligned}
\|\varphi\|_{\mathbb{B}} & \leqslant \sum_{n=-\infty}^{+\infty}\|\varphi(\eta) \psi(\eta-n)\|_{\mathbb{B}} \\
& \leqslant \sum_{n=-\infty}^{+\infty} \frac{M}{(1+|n|)^{2}}\left\|(1+|n|)^{2+Q} e^{\beta|n|} \varphi(\eta+n) \psi(\eta)\right\|_{\mathbb{B}} \\
& =\sum_{n=-\infty}^{+\infty} \frac{M}{(1+|n|)^{2}}\left\|g_{n}\right\|_{\mathbb{B}} \\
& \leqslant \sum_{n=-\infty}^{+\infty} \frac{M}{(1+|n|)^{2}} C_{6} P_{\beta, N_{0}+Q+2}[\varphi] \leqslant C_{7} P_{\beta, N_{0}+Q+2}[\varphi],
\end{aligned}
$$

что и доказывает (6.4). Плотность этого вложения следует из плотности вложения $\mathscr{D}$ в $\mathbb{B}$.

Если в аксиоме II

$$
\left\|T_{\xi}\right\| \leqslant M(1+|\xi|)^{Q}, \quad \xi \in \mathbb{R},
$$

для некоторых $M$ и $Q$, то пространство $\mathscr{S}$ плотно и непрерывно вложено в $\mathbb{B}$. Это следует из предыдущих рассуждений при $\beta=0$.

ТЕОРема 6.1. Пусть $\mathbb{B}$ - банахово пространство, для которого выполнены аксиомы I $u$ II, J - автомодельный функционал порядка $\alpha, g(\xi) \in \mathscr{D}^{\prime} u$ $\omega(\xi) \in \stackrel{n}{\mathscr{D} \backslash} \backslash_{\mathscr{D}}^{n+1}$ для некоторого челого $n$. Пусть функиия $\left(g(\xi), \frac{1}{y} \omega\left(\frac{\eta-\xi}{y}\right)\right) \in \mathbb{B}$ (как функция аргумента $\eta)$ при п.в. $y \in(0,1)$ и измерима по у как функция со значениями в $\mathbb{B}$, причем

$$
\hat{\mathbb{J}}\left(\left\|\left(g(\xi), \frac{1}{y} \omega\left(\frac{\eta-\xi}{y}\right)\right)\right\|_{\mathbb{B}}\right)<\infty .
$$


Пусть, кроме того, функиия $\omega_{0}(\xi) \in \mathscr{D} \backslash \stackrel{0}{\mathscr{D}}\left(\right.$ m.e. $\left.\int \omega_{0}(\xi) d \xi \neq 0\right)$ такая, что $\left(g(\xi), \omega_{0}(\eta-\xi)\right) \in \mathbb{B}$. Тогда отображение $\varphi \rightarrow(g(\xi), \varphi(\xi-\eta))$ является непрерывным линейным отображением из $\mathscr{D}$ в $\mathbb{B}($ т.е. принадлежит $\mathbb{L}(\mathscr{D} \rightarrow$ $\mathbb{B}))$ и для любого $а>0$ существуют $C$ и $N$ такие, что

$$
\begin{gathered}
\hat{\mathbb{J}}\left(\left\|\left(g(\xi), \frac{1}{y} \varphi\left(\frac{\eta-\xi}{y}\right)\right)\right\|_{\mathbb{B}}\right)+\left\|\left(g(\xi), \varphi_{0}(\eta-\xi)\right)\right\|_{\mathbb{B}} \\
\leqslant C\left[\hat{\mathbb{J}}\left(\left\|\left(g(\xi), \frac{1}{y} \omega\left(\frac{\eta-\xi}{y}\right)\right)\right\|_{\mathbb{B}}\right)+\left\|\left(g(\xi), \omega_{0}(\eta-\xi)\right)\right\|_{\mathbb{B}}\right]\left[\mathscr{P}_{N}[\varphi]+\mathscr{P}_{N}\left[\varphi_{0}\right]\right] \\
\forall \varphi \in \mathscr{D}_{a}, \quad \forall \varphi_{0} \in \mathscr{D}_{a},
\end{gathered}
$$

где $m=\min \{n,[-\alpha]\}$. Здесь функиии из пространства $\mathbb{B}$ имеют аргумент $\eta$, а автомодельный функиионал $\hat{\mathbb{J}}$ действует на функции аргумента у. Постоянные $C$ и $N$ не зависят от $g, \varphi$ и $\varphi_{0}$.

ДокАЗАТЕЛЬСТво. Введем (обобщеннозначную) обобщенную функцию $f \in$ $\mathbb{L}\left(\mathscr{D} \rightarrow \mathscr{D}^{\prime}\right)$ по формуле

$$
(f(\xi), \varphi(\xi))(\eta)=(g(\xi), \varphi(\xi-\eta)) \quad \forall \varphi \in \mathscr{D} .
$$

Замечая, что

$$
L_{f}^{\omega}(x, y)=\left(f(\xi), \frac{1}{y} \omega\left(\frac{x-\xi}{y}\right)\right)=\left(g(\xi), \frac{1}{y} \omega\left(\frac{x+\eta-\xi}{y}\right)\right)
$$

и, в частности, $L_{f}^{\omega}(0,1)=(g(\xi), \omega(\eta-\xi))$, легко проверить, что выполнены все условия теоремы 4.1. Поэтому отображение $\varphi \mapsto(g(\xi), \varphi(\xi-\eta))$ принадлежит $\mathbb{L}(\mathscr{D} \rightarrow \mathbb{B})$. Из замечания 4.1 (см. (4.9)) следует оценка (6.7). Теорема доказана.

ЗАмЕчАниЕ 6.1. Оценку (6.7), учитывая (3.1), можно переписать следующим образом: для любого $a>0$ существуют $C$ и $N$ такие, что

$$
\begin{aligned}
\hat{\mathbb{J}}\left(\left\|L_{g}^{\varphi}(\eta, y)\right\|_{\mathbb{B}}\right)+\left\|L_{g}^{\varphi_{0}}(\eta, 1)\right\|_{\mathbb{B}} \leqslant C\left[\hat{\mathbb{J}}\left(\left\|L_{g}^{\omega}(\eta, y)\right\|_{\mathbb{B}}\right)+\left\|L_{g}^{\omega_{0}}(\eta, 1)\right\|_{\mathbb{B}}\right] \\
\times\left[\mathscr{P}_{N}[\varphi]+\mathscr{P}_{N}\left[\varphi_{0}\right]\right] \quad \forall \varphi \in \stackrel{m}{D}_{a}, \quad \forall \varphi_{0} \in \mathscr{D}_{a}
\end{aligned}
$$

где $C$ и $N$ не зависят от $g, \varphi$ и $\varphi_{0}$.

Введем обозначение

$$
\|g(\xi)\|_{V_{\mathbb{B}}^{\mathbb{J}, \omega, \omega_{0}}}=\hat{\mathbb{J}}\left(\left\|\left(g(\xi), \frac{1}{y} \omega\left(\frac{\eta-\xi}{y}\right)\right)\right\|_{\mathbb{B}}\right)+\left\|\left(g(\xi), \omega_{0}(\eta-\xi)\right)\right\|_{\mathbb{B}} .
$$

Если $g \in \mathscr{D}^{\prime}$ и $\omega, \omega_{0}$ удовлетворяют условиям теоремы 6.1 , то для любого $a>0$ сушествуют $C$ и $N$ такие, что

$$
\|g(\xi)\|_{V_{\mathbb{B}}^{\mathbb{J}, \varphi, \varphi_{0}}} \leqslant C\left[\mathscr{P}_{N}[\varphi]+\mathscr{P}_{N}\left[\varphi_{0}\right]\right]\|g(\xi)\|_{V_{\mathbb{B}}^{\mathbb{J}, \omega, \omega_{0}}} \quad \forall \varphi \in \stackrel{m}{\mathscr{D}}_{a}, \quad \forall \varphi_{0} \in \mathscr{D}_{a} .
$$


ОПредЕлениЕ 6.1 . Обозначим через $V_{\mathbb{B}}^{\mathbb{J}, \omega, \omega_{0}}$ множество тех $g \in \mathscr{D}^{\prime}$, для которых $\left(g(\xi), \frac{1}{y} \omega\left(\frac{\eta-\xi}{y}\right)\right) \in \mathbb{B}$ при $0<y \leqslant 1$, а также $\left(g(\xi), \omega_{0}(\eta-\xi)\right) \in \mathbb{B}$, и для которых $\|g\|_{V_{\mathbb{B}}^{\mathrm{J}, \omega, \omega_{0}}}<\infty$.

Отметим, что $\|\cdot\|_{V_{\mathbb{B}}^{\mathrm{J}, \omega, \omega_{0}}}-$ полунорма.

УТВЕРЖДЕНИЕ 6.1. $\|\cdot\|_{V_{\mathbb{B}}^{\mathrm{J}, \omega, \omega_{0}}}-$ норм⿱ и отображение $V_{\mathbb{B}}^{\mathbb{J}, \omega, \omega_{0}} \rightarrow \mathscr{D}^{\prime}$ непрерывно.

ДокАЗАТЕЛЬСТво. Фиксируем $\varphi_{0}$ и $\gamma$, принадлежашие $\mathscr{D}_{[-1,1]} \backslash \stackrel{0}{\mathscr{D}}$, и обозначим $W=\varphi_{0} * \gamma$. Оценим $\left|L_{g}^{W}(x, y)\right|$. Имеем

$$
\begin{aligned}
\left|L_{g}^{W}(x, y)\right| & =\left|\left(g(\xi), \frac{1}{y} W\left(\frac{x-\xi}{y}\right)\right)\right|=\left|\left(g(\xi), \int \frac{1}{y} \gamma\left(\frac{\eta}{y}\right) \frac{1}{y} \varphi_{0}\left(\frac{\eta-\xi+x}{y}\right) d \eta\right)\right| \\
& \leqslant\left|\int \frac{1}{y} \gamma\left(\frac{\eta}{y}\right)\left(g(\xi), \frac{1}{y} \varphi_{0}\left(\frac{\eta-\xi+x}{y}\right) d \eta\right)\right| \\
& \leqslant\left\|\frac{1}{y} \gamma\left(\frac{\eta}{y}\right)\right\|_{\mathbb{B}^{*}}\left\|\left(g(\xi), \frac{1}{y} \varphi_{0}\left(\frac{\eta-\xi+x}{y}\right)\right)\right\|_{\mathbb{B}} .
\end{aligned}
$$

В силу первого вложения (6.3) $\|\gamma\|_{\mathbb{B}^{*}} \leqslant A \mathscr{P}_{M}[\gamma]$ при $\gamma \in \mathscr{D}_{[-1,1]}$ для некоторых $A$ и $M$. Из (6.8) при $\varphi_{0}=\psi, \varphi=0$ вытекает, что для любого $a>0$ найдутся $C$ и $N$ такие, что

$$
\left\|L_{g}^{\psi}(\eta, 1)\right\|_{\mathbb{B}}=\|(g(\xi), \psi(\eta-\xi))\|_{\mathbb{B}} \leqslant C \mathscr{P}_{N}[\psi]\|g\|_{V_{\mathbb{B}}^{\mathrm{J}, \omega, \omega}, \omega_{0}}
$$

где $\psi \in \mathscr{D}_{a} \backslash \stackrel{0}{\mathscr{D}}$. В частности, для любого $a_{1}$ найдутся $C_{1}$ и $N_{1}$ такие, что

$$
\left\|\left(g(\xi), \frac{1}{y} \varphi_{0}\left(\frac{\eta-\xi+x}{y}\right)\right)\right\|_{\mathbb{B}} \leqslant C_{1} \mathscr{P}_{N_{1}}\left[\frac{1}{y} \varphi_{0}\left(\frac{\xi-x}{y}\right)\right]\|g\|_{V_{\mathbb{B}}^{\mathrm{J}}, \omega, \omega_{0}}
$$

при $|x|<a_{1}$. Учитьвая эти неравенства и продолжая оценку для $\left|L_{g}^{W}(x, y)\right|$, получим, что для любого $a_{1}$ найдутся $C_{1}$ и $N_{1}$ такие, что

$$
\begin{aligned}
\left|L_{g}^{W}(x, y)\right| & =\left|\left(g(\xi), \frac{1}{y} W\left(\frac{\xi-x}{y}\right)\right)\right| \\
& \leqslant A C_{1} \mathscr{P}_{M}\left[\frac{1}{y} \gamma\left(\frac{\eta}{y}\right)\right] \mathscr{P}_{N_{1}}\left[\frac{1}{y} \varphi_{0}\left(\frac{\eta+x}{y}\right)\right]\|g\|_{V_{\mathbb{B}}^{\mathrm{J}, \omega, \omega}} \\
& \leqslant \frac{A_{1}}{y^{M+N+4}}\|g\|_{V_{\mathbb{B}}^{\mathrm{J}, \omega, \omega_{0}}} .
\end{aligned}
$$

Применяя теорему 3.1 для $\mathbb{J}(\cdot)=\sup (\cdot)$ и $\mathbb{B}=\mathbb{C}$, получим, что для любого $a_{2}$ сушествуют $C_{2}$ и $N_{2}$ такие, что

$$
|(g(\xi), \varphi(\xi))|<C_{2} \mathscr{P}_{N_{2}}[\varphi]\|g\|_{V_{\mathbb{B}}^{\mathrm{J}, \omega, \omega} 0} \quad \forall \varphi \in \mathscr{D}_{a_{2}}
$$

что и доказывает утверждение. 
УТВЕРЖДЕНИЕ 6.2. Пространство $V_{\mathbb{B}}^{\mathbb{J}, \omega, \omega_{0}}$ банахово.

ДоКАЗАТЕЛЬСТВо. Согласно утверждению 6.1 осталось доказать только полноту пространства $V_{\mathbb{B}}^{\mathbb{J}, \omega, \omega_{0}}$. Пусть $f_{n}(\xi)$ - фундаментальная последовательность в $V_{\mathbb{B}}^{\mathbb{J}, \omega, \omega_{0}}$, т.е.

$$
\left\|f_{n}-f_{m}\right\|_{V_{\mathbb{B}}^{\mathbb{J}, \omega, \omega_{0}}} \rightarrow 0 \text { при } n, m \rightarrow+\infty .
$$

Из предыдущего утверждения и полноты $\mathscr{D}^{\prime}$ следует, что существует $f_{\infty}(\xi) \in \mathscr{D}^{\prime}$ такая, что

$$
f_{n}(\xi) \underset{n \rightarrow \infty}{\longrightarrow} f_{\infty}(\xi) \text { в } \mathscr{D}^{\prime} .
$$

Докажем, что $f_{n}(\xi) \underset{n \rightarrow \infty}{\longrightarrow} f_{\infty}(\xi)$ в $V_{\mathbb{B}}^{\mathbb{I}, \omega, \omega_{0}}$.

Фундаментальность последовательности по первой части нормы $\|\cdot\|_{V_{\mathbb{B}}^{\rrbracket}, \omega, \omega_{0}}$ (см. (6.9)) означает, что последовательность $L_{f_{n}}^{\omega}(\cdot, y)$ фундаментальна в пространстве $\mathbb{L}^{\hat{J}}((0,1) \rightarrow \mathbb{B})$ (см. замечание 2.1$)$. В силу полноты этого пространства существует функция $G_{y}(\cdot) \in \mathbb{L}^{\hat{\mathbb{J}}}((0,1) \rightarrow \mathbb{B})$ такая, что

$$
L_{f_{n}}^{\omega}(\cdot, y) \underset{n \rightarrow \infty}{\longrightarrow} G_{y}(\cdot) \quad \text { в } \quad \mathbb{L}^{\hat{\mathbb{J}}}((0,1) \rightarrow \mathbb{B})
$$

Следовательно, существует подпоследовательность $L_{f_{n_{k}}}^{\omega}(\eta, y) \underset{k \rightarrow+\infty}{\longrightarrow} G_{y}(\eta)$ почти всюду по $y \in(0,1)$. С другой стороны, из (6.11) вытекает, что

$$
\begin{aligned}
L_{f_{n_{k}}}^{\omega}(\eta, y) & =\left(f_{n_{k}}(\xi), \frac{1}{y} \omega\left(\frac{\eta-\xi}{y}\right)\right) \underset{k \rightarrow+\infty}{\longrightarrow} \\
& \underset{k \rightarrow+\infty}{\longrightarrow}\left(f_{\infty}(\xi), \frac{1}{y} \omega\left(\frac{\eta-\xi}{y}\right)\right)=L_{f_{\infty}}^{\omega}(\eta, y) \text { в } \mathscr{D}^{\prime}
\end{aligned}
$$

(более того, даже равномерно на компактах по $\eta$ при всех $y \in(0,1)$ ). Итак, последовательность $L_{f_{n_{k}}}^{\omega}(\eta, y)$ при п.в. $y \in(0,1)$ сходится к $G_{y}(\eta)$ в $\mathbb{B}$ и при всех $y \in(0,1]$ сходится к $L_{f_{\infty}}^{\omega}(\eta, y)$ в $\mathscr{D}^{\prime}$. Отсюда следует, что $G_{y}(\eta)=L_{f_{\infty}}^{\omega}(\eta, y)$ при п.в. $y \in(0,1)$, и, следовательно,

$$
L_{f_{n}}^{\omega}(\eta, y) \underset{n \rightarrow \infty}{\longrightarrow} L_{f_{\infty}}^{\omega}(\eta, y) \text { в } \mathbb{L}^{\hat{\mathbb{N}}}((0,1) \rightarrow \mathbb{B}) .
$$

Рассмотрим вторую часть нормы $\|\cdot\|_{V_{\mathbb{B}}^{\mathbb{J}, \omega, \omega_{0}}}$. По условию $L_{f_{n}}^{\omega_{0}}(\eta, 1)$ фундаментальна в $\mathbb{B}$, поэтому существует $F(\eta) \in \mathbb{B}$ такая, что $L_{f_{n}}^{\omega_{0}}(\eta, 1) \underset{n \rightarrow \infty}{\longrightarrow} F(\eta)$ в $\mathbb{B}$. В силу $(6.11) L_{f_{n}}^{\omega_{0}}(\eta, 1) \underset{n \rightarrow \infty}{\longrightarrow} L_{f_{\infty}}^{\omega_{0}}(\eta, 1)$ в $\mathscr{D}^{\prime}$, а поэтому $F(\eta)=L_{f_{\infty}}^{\omega_{0}^{\infty}}(\eta, 1)$ в $\mathscr{D}^{\prime}$, а стало быть, и в $\mathbb{B}$. Таким образом,

$$
L_{f_{n}}^{\omega_{0}}(\eta, 1) \underset{n \rightarrow \infty}{\longrightarrow} L_{f_{\infty}}^{\omega_{0}}(\eta, 1) \text { в } \mathbb{B} .
$$

Из (6.12) и (6.13) следует, что $f_{\infty}$ есть предельная функция нашей фундаментальной последовательности в $V_{\mathbb{B}}^{\mathbb{J}, \omega, \omega_{0}}$. Утверждение доказано. 
УТВЕРЖДЕНИЕ 6.3. Пусть $\mathbb{J}-$ автомодельный функционал порядка $\alpha$ и $\mathbb{B}$ - банахово пространство с аксиомами I, II. Для челых $n=-1,0,1,2, \ldots$ рассмотрим следующие случаи ${ }^{2}$.

1) $n \geqslant[-\alpha]$. Тогда все пространства $V_{\mathbb{B}}^{\mathbb{J}, \varphi, \varphi_{0}}$ для любых различных $\varphi_{0} \in$ $\mathscr{D} \backslash \stackrel{0}{\mathscr{D}}$ и любых различных $\varphi \in \stackrel{n}{\mathscr{D}}, \varphi \not \equiv 0$, совпадают как множества, причем все нормы $\|\cdot\|_{V_{\mathbb{B}}^{\mathrm{J}, \varphi, \varphi_{0}}}$ әквивалентны. Тем самым банаховы пространства $V_{\mathbb{B}}^{\mathbb{J}, \varphi, \varphi_{0}}$ с указанньми свойствами $\varphi_{0}$ и $\varphi$ определяют с точностью до әквивалентности норм одно и то же банахово пространство, которое будем обозначать $V_{\mathbb{B}}^{\mathbb{J}}$.

2) $n<[-\alpha]$. Возможны два подслучая:

а) $n=-\alpha-1$ (это возможнно только при $\alpha=0,-1,-2, \ldots$ ); тогда для любых различных $\varphi_{0} \in \mathscr{D} \backslash \stackrel{0}{\mathscr{D}}$ и любых различных $\varphi \in \stackrel{n}{\mathscr{D} \backslash} \backslash \stackrel{n+1}{\mathscr{D}}$ пространства $V_{\mathbb{B}}^{\mathbb{J}, \varphi, \varphi_{0}}$ әквивалентны, т.е. с точностью до әквивалентности норм определяют одно и то же банахово пространство, которое будем обозначать $\widehat{V}_{\mathbb{B}}^{\mathbb{J}}$;

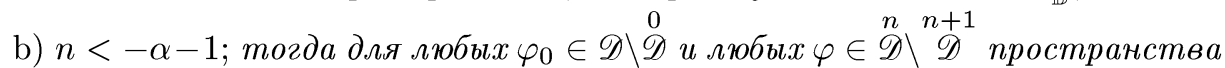
$V_{\mathbb{B}}^{\mathbb{J}, \varphi, \varphi_{0}}$ тривиальны в том смысле, что они состоят из полиномов степени не выше $n$, содержащихся в $\mathbb{B}$.

ДокАЗАТЕльСтво. Случаи 1) и 2, a) вытекают из теоремы 6.1 и замечания 6.1 (в этих случаях в соотношении (6.10) $\omega, \omega_{0}$ и $\varphi, \varphi_{0}$ можно поменять местами). Остается доказать тривиальность пространств $V_{\mathbb{B}}^{\mathbb{J}, \varphi, \varphi_{0}}$ в случае $\left.2, \mathrm{~b}\right)$.

Действительно, пусть $g \in V_{\mathbb{B}}^{\mathbb{J}, \varphi, \varphi_{0}}$, где $\varphi_{0} \in \mathscr{D} \backslash \stackrel{0}{\mathscr{D}}, \varphi \in \stackrel{n}{\mathscr{D}} \backslash \stackrel{n+1}{\mathscr{D}}$ при $n<-\alpha-1$. Так как $\mathbb{B} \subset \mathscr{D}^{\prime}$ непрерывно, то для любого $\psi \in \mathscr{D}$ имеем

$$
\left|\int\left(g(\xi), \frac{1}{y} \varphi\left(\frac{\eta-\xi}{y}\right)\right) \psi(\eta) d \eta\right| \leqslant\|\psi\|_{\mathbb{B}^{*}}\left\|\left(g(\xi), \frac{1}{y} \varphi\left(\frac{\eta-\xi}{y}\right)\right)\right\|_{\mathbb{B}} .
$$

Согласно (2.9) после замены переменных $k=\frac{1}{y}$ получим

$$
\begin{aligned}
& \int_{0}^{1} y^{\alpha+\varepsilon}\left|\int\left(g(\xi), \frac{1}{y} \varphi\left(\frac{\eta-\xi}{y}\right)\right) \psi(\eta) d \eta\right| \frac{d y}{y} \leqslant \\
& \leqslant C_{\varepsilon}\|\psi\|_{\mathbb{B}^{*} \hat{J}}\left(\left\|\left(g(\xi), \frac{1}{y} \varphi\left(\frac{\eta-\xi}{y}\right)\right)\right\| \|_{\mathbb{B}}\right)<\infty .
\end{aligned}
$$

Отсюда, учитывая, что $\varphi(\xi)=\varphi_{1}^{(n+1)}(\xi)$, где $\int \varphi_{1}(\xi) d \xi=C_{1} \neq 0$, и, следовательно,

$$
\int\left(g(\xi), \frac{1}{y} \varphi\left(\frac{\eta-\xi}{y}\right)\right) \psi(\eta) d \eta=y^{n+1} \int\left(g^{(n+1)}(\xi), \frac{1}{y} \varphi_{1}\left(\frac{\eta-\xi}{y}\right)\right) \psi(\eta) d \eta,
$$

имеем

$$
\int_{0}^{1} y^{\alpha+\varepsilon+n+1}\left|\int\left(g^{(n+1)}(\xi), \frac{1}{y} \varphi_{1}\left(\frac{\eta-\xi}{y}\right)\right) \psi(\eta) d \eta\right| \frac{d y}{y}<\infty .
$$

\footnotetext{
${ }^{2}$ Напомним, что по нашему соглашению $\stackrel{-1}{\mathscr{D}}=\mathscr{D}$.
} 
Замечая, что

$$
\int\left(g^{(n+1)}(\xi), \frac{1}{y} \varphi_{1}\left(\frac{\eta-\xi}{y}\right)\right) \psi(\eta) d \eta \underset{y \rightarrow+0}{\longrightarrow} C_{1}\left(g^{(n+1)}(\xi), \psi(\xi)\right),
$$

а $\alpha+\varepsilon+n+1<0$ при достаточно малом $\varepsilon$, из (6.14) выводим, что

$$
\left(g^{(n+1)}(\xi), \psi(\xi)\right)=0 \quad \forall \psi \in \mathscr{D}
$$

т.е. $g(\xi)$ - многочлен степени не выше $n$. Утверждение доказано.

ЗАмЕчАниЕ 6.2. Пространства $V_{\mathbb{B}}^{\mathbb{J}}$ (случай 1$)$ ) всегда нетривиальны, так как

$$
E_{\beta} \subset V_{\mathbb{B}}^{\mathbb{J}}
$$

где $\beta$ берется из соотношения (6.2), причем вложение непрерывно.

Действительно, пусть $f \in E_{\beta}$ и $\omega \in \stackrel{n}{\mathscr{D}} \backslash_{\mathscr{D}}^{n+1}, n>-\alpha$, где $\alpha$ - порядок автомодельности J. Учитывая, что $\omega=\omega_{1}^{(n)}$, где $\omega_{1} \in \mathscr{D} \backslash \stackrel{0}{\mathscr{D}}, \operatorname{supp} \omega_{1} \subset[-1,1]$, $\int \omega_{1}(\xi) d \xi \neq 0$ (без ограничения общности можно считать, что $\int \omega_{1}(\xi) d \xi=1$ ), и пользуясь $(2.9)$, имеем

$$
\begin{aligned}
\hat{\mathbb{J}}\left(\left\|L_{f}^{\omega}(x, y)\right\|_{\mathbb{B}}\right) & =\hat{\mathbb{J}}\left(\left\|\left(f(\xi), \frac{1}{y} \omega_{1}^{(n)}\left(\frac{x-\xi}{y}\right)\right)\right\|_{\mathbb{B}}\right) \\
& =\hat{\mathbb{J}}\left(y^{n}\left\|\left(f^{(n)}(\xi), \frac{1}{y} \omega_{1}\left(\frac{x-\xi}{y}\right)\right)\right\|_{\mathbb{B}}\right) \\
& \leqslant C_{\varepsilon} \sup _{0<y \leqslant 1} y^{\alpha-\varepsilon+n}\left\|\left(f^{(n)}(\xi), \frac{1}{y} \omega_{1}\left(\frac{x-\xi}{y}\right)\right)\right\|_{\mathbb{B}} \\
& \leqslant C_{\varepsilon} \sup _{0<y \leqslant 1} P_{\beta, N}\left[\left(f^{(n)}(\xi), \frac{1}{y} \omega_{1}\left(\frac{x-\xi}{y}\right)\right)\right] .
\end{aligned}
$$

В последнем неравенстве мы воспользовались тем, что $\alpha-\varepsilon+n>0$, и непрерывностью вложения $E_{\beta}$ в $\mathbb{B}$ (см. (6.4)). Учитывая, что

$$
\begin{aligned}
P_{\beta, N}[ & \left.\left(f^{(n)}(\xi), \frac{1}{y} \omega_{1}\left(\frac{x-\xi}{y}\right)\right)\right] \\
& =\max _{j \leqslant N} \sup _{x}(1+|x|)^{N} e^{\beta|x|}\left|\int\left(f^{(j+n)}(\xi) \frac{1}{y} \omega_{1}\left(\frac{x-\xi}{y}\right)\right) d \xi\right| \\
& \leqslant \max _{j \leqslant N} \sup _{x}(1+|x|)^{N} e^{\beta|x|} \sup _{x-1<\xi<x+1}\left|f^{(j+n)}(\xi)\right| \\
& =\max _{j \leqslant N} \sup _{\xi}\left|f^{(j+n)}(\xi)\right| \sup _{\xi-1<x<\xi+1}(1+|x|)^{N} e^{\beta|x|} \\
& \leqslant \max _{j \leqslant N} \sup _{\xi}\left|f^{(j+n)}(\xi)(2+|\xi|)^{N} e^{\beta(|\xi|+1)}\right| \leqslant C_{1} P_{\beta, N+n}[f],
\end{aligned}
$$

из (6.16) получаем, что $\hat{\mathbb{J}}\left(\left\|L_{f}^{\omega}(x, y)\right\|_{\mathbb{B}}\right) \leqslant C_{2} P_{\beta, N+n}[f]$. Вторая часть нормы $V_{\mathbb{B}}^{\mathbb{J}}$ оценивается аналогично (проще), что и доказывает (6.15). 
Пространства $\widehat{V}_{\mathbb{B}}^{J}($ случай 2, а)) в зависимости от автомодельного функционала J может быть тривиальным (конечномерным) и нетривиальным.

Из определений пространств непосредственно следует, что

$$
\widehat{V}_{\mathbb{B}}^{\mathbb{J}} \subset V_{\mathbb{B}}^{\mathbb{J}}
$$

Например, пространство $V_{\mathbb{B}}^{\mathbb{J}^{\infty}}$ состоит из обобщенных функций $f(\xi) \in \mathscr{D}^{\prime}$ таких, что

$$
\sup _{0<y \leqslant 1}\left\|\left(f(\xi), \frac{1}{y} \omega\left(\frac{\eta-\xi}{y}\right)\right)\right\|_{\mathbb{B}}<\infty, \quad\left(f(\xi), \omega_{0}(\eta-\xi)\right) \in \mathbb{B},
$$

где $\omega \neq \equiv 0, \int \omega(\xi) d \xi=0$ и $\int \omega_{0}(\xi) d \xi \neq 0$. Пространство $\widehat{V}_{\mathbb{B}}^{\rrbracket^{\infty}}$ состоит из обобщенных функций $f \in \mathscr{D}^{\prime}$, для которых выполнены те же соотношения, но с $\omega$ такой, что $\int \omega(\xi) d \xi \neq 0$.

Вложение (6.17), вообще говоря, строгое. Действительно, положим $\mathbb{J}(\cdot)=$ $\mathbb{J}^{\infty}(\cdot)$ и $\mathbb{B}=C_{0}(\mathbb{R})$. Пространства $\widehat{V}_{C_{0}}^{\mathbb{J}^{\infty}}$ и $V_{C_{0}}^{\mathbb{J}^{\infty}}$ не пусты, так как содержат $\mathscr{D}$. В то же время можно показать, что функщия $h(\xi) \ln |\xi|$, где $h(\xi) \in \mathscr{D}$ и $h(0)=1$, принадлежит $V_{C_{0}}^{\mathbb{J}^{\infty}}$, но не принадлежит $\widehat{V}_{C_{0}}^{\mathbb{J}^{\infty}}$.

Отметим, что в $V_{\mathbb{B}}^{\mathbb{J}}$ определена группа сдвигов $\left\{T_{a} \in L\left(V_{\mathbb{B}}^{\mathbb{J}} \rightarrow V_{\mathbb{B}}^{\mathbb{J}}\right), a \in \mathbb{R}\right\}$, удовлетворяюшая всем условиям аксиомы II, в частности,

$$
\left\|T_{a} f\right\|_{V_{\mathbb{B}}^{\mathrm{J}}} \leqslant C(a)\|f\|_{V_{\mathbb{B}}^{\mathrm{J}}}, \quad a \in \mathbb{R},
$$

где $C(a)$ берется из формулы (6.1).

ЛЕмма 6.1. Пространство $V_{\mathbb{B}}^{\mathbb{J}}$ непрерывно вложено в $E_{\beta}^{\prime}$, где $\beta$ берется из формуль (6.2).

ДокАЗАТЕЛЬСТво. Пусть $f \in V_{\mathbb{B}}^{\mathbb{J}}$, из утверждения 6.1 вытекает, что существуют $C$ и $N$ такие, что

$$
|(f(\xi), \phi(\xi))| \leqslant C\|f\|_{V_{\mathbb{B}}^{\rrbracket}} \mathscr{P}_{N}[\phi] \quad \forall \phi \in \mathscr{D}_{[-1,+1]}
$$

Отсюда следует, что

$$
|(f(\xi+a), \phi(\xi))| \leqslant C\|f(\xi+a)\|_{V_{\mathbb{B}}^{\text {J }}} \mathscr{P}_{N}[\phi] \leqslant C M(1+|\alpha|)^{Q} e^{\beta|a|}\|f\|_{V_{\mathbb{B}}^{\text {J }}} \mathscr{P}_{N}[\phi] .
$$

Теперь воспользуемся специальным разбиением еденицы (6.5). Для любой функции $\varphi \in E_{\beta}$ имеем

$$
(f(\xi), \varphi(\xi))=\sum_{n=-\infty}^{+\infty}(f(\xi), \psi(\xi-n) \varphi(\xi))=\sum_{n=-\infty}^{+\infty}(f(\xi+n), \psi(\xi) \varphi(\xi+n)) .
$$


Оценим отдельные слагаемые этой суммы:

$$
\begin{aligned}
& |(f(\xi+n), \psi(\xi) \varphi(\xi+n))| \leqslant M(1+|n|)^{Q} e^{\beta|n|}\|f\|_{V_{\mathbb{B}}^{\mathbb{J}}} \mathscr{P}_{N}[\psi(\xi) \varphi(\xi+n)]
\end{aligned}
$$

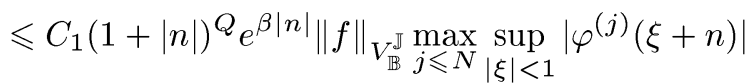

$$
\begin{aligned}
& =\frac{C_{2}}{(1+|n|)^{2}}\|f\|_{V_{\mathbb{B}}^{\mathbb{J}}} \max _{j \leqslant N} \sup _{|\eta-n|<1} e^{\beta|n|}(1+|n|)^{2+Q}\left|\varphi^{(j)}(\eta)\right|
\end{aligned}
$$

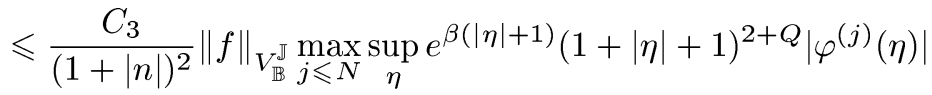

$$
\begin{aligned}
& \leqslant \frac{C_{4}}{(1+|n|)^{2}}\|f\|_{V_{\mathbb{B}}^{\rrbracket}} P_{\beta, N+Q+2}[\varphi] .
\end{aligned}
$$

Отсюда выводим, что для любой функции $\varphi \in E_{\beta}$

$$
|(f, \varphi)| \leqslant \sum_{n=-\infty}^{+\infty} \frac{C_{5}}{(1+|n|)^{2}}\|f\|_{V_{\mathbb{B}}^{\mathbb{J}}} P_{\beta, N+Q+2}[\varphi] \leqslant C_{6}\|f\|_{V_{\mathbb{B}}^{\mathbb{J}}} P_{\beta, N+Q+2}[\varphi],
$$

откуда и вытекает утверждение леммы.

ЗАмеЧАнИЕ 6.3. Пусть в аксиоме II для некоторых $M$ и $Q$

$$
\left\|T_{a}\right\| \leqslant M(1+|a|)^{Q}, \quad-\infty<a<+\infty .
$$

Тогда $V_{\mathbb{B}}^{\mathbb{J}}$ непрерьвно вложено в $\mathscr{S}^{\prime}$. Это утверждение вытекает из леммы 6.1 при $\beta=0$.

Далее будем предполагать, что наряду с аксиомами I и II выполнена также и следуюшая аксиома.

III. B $\mathbb{B}$ определен оператор умножения на функции из $C_{0}^{\infty}(\mathbb{R})$, причем отображение $C_{0}^{\infty} \mapsto \mathbb{L}(\mathbb{B} \rightarrow \mathbb{B})$ непрерывно, т.е. существуют постоянные $C$ и $N$ такие, что

$$
\|\eta(\xi) g(\xi)\|_{\mathbb{B}} \leqslant C Q_{N}[\eta]\|g\|_{\mathbb{B}} \quad \forall \eta \in C_{0}^{\infty}
$$

(см. (1.11)).

СлеДСТвИЕ 6.1. Существует постоянная $A$ такая, что для любого $а>0$ существует функция $\eta(\xi) \in \mathscr{D}, \quad \eta(\xi) \equiv 1$ при $|\xi| \leqslant a$, такая, что

$$
\|\eta(\xi) g(\xi)\|_{\mathbb{B}} \leqslant A\|g\|_{\mathbb{B}} \quad \forall g \in \mathbb{B} .
$$

ЗАмеЧАнИЕ 6.4. Из (6.21) вытекает, что

$$
|(\eta(\xi) f(\xi), g(\xi))|=|(f(\xi), \eta(\xi) g(\xi))| \leqslant\|f\|_{\mathbb{B}^{*}}\|\eta g\|_{\mathbb{B}} \leqslant A\|f\|_{\mathbb{B}^{*}}\|g\|_{\mathbb{B}}
$$

Таким образом, сушествует постоянная $A$ такая, что для любого $a>0$ существует функция $\eta(\xi) \in \mathscr{D}, \eta(\xi) \equiv 1$ при $|\xi| \leqslant a$, такая, что

$$
\|\eta(\xi) f(\xi)\|_{\mathbb{B}^{*}} \leqslant A\|f\|_{\mathbb{B}^{*}} \quad \forall f \in \mathbb{B}^{*} .
$$


Далее мы исследуем некоторые свойства введенных банаховых пространств, вытекающие из аксиом I-III.

Введем в $\mathbb{B}$ новую норму $\mathscr{P}[\cdot]$ по формуле

$$
\mathscr{P}[g]=\sup _{\substack{\varphi \in \mathscr{D} \\\|\varphi\|_{\mathbb{B}^{*} \leqslant 1}}}|(g(\xi), \varphi(\xi))|, \quad g \in \mathbb{B},
$$

где $(g(\xi), \varphi(\xi))$ - значение обобшенной функции $g$ на основной функции $\varphi$. Извест-

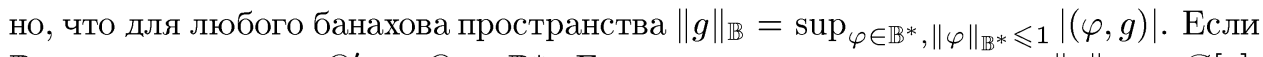
$\mathbb{B}$ плотно вложено в $\mathscr{D}^{\prime}$, то $\mathscr{D} \subset \mathbb{B}^{*}$. Если это вложение плотно, то $\|g\|_{\mathbb{B}}=\mathscr{P}[g]$. Если же оно не плотно, то можно лишь утверждать, что $\mathscr{P}[g] \leqslant\|g\|_{\mathbb{B} .}$ Однако если выполнены аксиомы I-III, то эти нормы эквивалентны. Точнее, справедлива следуюшая

ЛЕмма 6.2. Пусть $\mathbb{B}$ - банахово пространство, для которого выполнены аксиомы I-III; тогда $\|\cdot\|_{\mathbb{B}} \sim \mathscr{P}[\cdot]$, при этом

$$
\mathscr{P}[g] \leqslant\|g\|_{\mathbb{B}} \leqslant M A \mathscr{P}[g] \quad \forall g \in \mathbb{B},
$$

где постоянные $M$ и А определяются из формуль (6.2) и следствия 6.1.

ДокАЗАТЕЛЬСтво. Достаточно доказать лишь второе неравенство (6.22). Определим в $\mathbb{B}^{*}$ топологию $\tau$ системой полунорм $\left\{P_{w}[f]=|(f, w)|, w \in \mathscr{D}\right\}$. Обозначим $U_{r}=\left\{f \in \mathbb{B}^{*},\|f\|_{\mathbb{B}^{*}}<r\right\}$, ипусть $\delta>0$. Сперва покажем, что $\mathscr{D} \cap U_{A(M+\delta)}$ плотно в $U_{1}$ в топологии $\tau$.

Действительно, пусть $f \in U_{1}$, зафиксированы $w_{1}, w_{2}, \ldots, w_{n}$ из $\mathscr{D}$ и $\varepsilon>0$. Возьмем "шапочку" $W(\xi) \in \mathscr{D}, W \geqslant 0, \operatorname{supp} W \subset[-1,1], \int W(\xi) d \xi=1$, и положим $\varphi_{k}(\xi)=\eta(\xi)\left(f * W_{k}\right)$, где $W_{k}(\xi)=k W(k \xi)$, а функция $\eta(\xi) \in \mathscr{D}$ будет подобрана позднее. Считаем, что $\eta \equiv 1$ на $\operatorname{supp} w_{i}, i=1, \ldots, n$. Тогда существует $k_{\varepsilon}$ такое, что

$$
P_{w_{i}}\left[f-\varphi_{k}\right]=\left|\left(f-\eta\left(f * W_{k}\right), w_{i}\right)\right|=\left|\left(f-f * W_{k}, w_{i}\right)\right|<\varepsilon, \quad i=1, \ldots, n,
$$

при $k>k_{\varepsilon}$, так как $f * W_{k} \rightarrow f$ в $\mathscr{D}^{\prime}$. Оценим норму функционала $f * W_{k}$ в $\mathbb{B}^{*}$. Имеем

$$
\left(f * W_{k}, g\right)=\int(f(x), g(x-\xi)) W_{k}(\xi) d \xi=\int\left(f, T_{\xi} g\right) W_{k}(\xi) d \xi .
$$

Отсюда

$$
\begin{aligned}
\left\|f * W_{k}\right\|_{\mathbb{B}^{*}} & =\sup _{\|g\|_{\mathbb{B}} \leqslant 1}\left|\int\left(f, T_{\xi} g\right) W_{k}(\xi) d \xi\right| \\
& \leqslant \sup _{\|g\|_{\mathbb{B}} \leqslant 1} \sup _{\xi \in \operatorname{supp} W_{k}}\left\|T_{\xi} g\right\|_{\mathbb{B}}\|f\|_{\mathbb{B}^{*}} \int W_{k}(\xi) d \xi \\
& \leqslant \sup _{\xi \in \operatorname{supp} W_{k}} C(\xi) \leqslant M+\delta
\end{aligned}
$$


при достаточно большом $k$ (мы учли, что $\left.\operatorname{supp} W_{k} \subset\left[-\frac{1}{k},+\frac{1}{k}\right]\right)$. Согласно замечанию 6.4 выбираем $\eta$ таким, чтобы $\left\|\varphi_{k}\right\|_{\mathbb{B}^{*}}=\left\|\eta\left(f * W_{k}\right)\right\| \leqslant A(M+\delta)$. Таким образом, плотность $\mathscr{D} \cap U_{A(M+\delta)}$ в $U_{1}$ в топологии $\tau$ доказана.

Пусть $g \in \mathbb{B}$. Так как $\mathscr{D}$ плотно вложено в $\mathbb{B}$, то для любого $\varepsilon>0$ существует $w \in \mathscr{D}$ такое, что $\|g-w\|_{\mathbb{B}}<\varepsilon$. Отсюда имеем

$$
\|g\|_{\mathbb{B}} \leqslant\|w\|_{\mathbb{B}}+\varepsilon=\varepsilon+\sup _{\|f\|_{\mathbb{B}^{*}} \leqslant 1}|(f, w)| \leqslant 2 \varepsilon+\left|\left(f_{0}, w\right)\right|
$$

для некоторого $\left\|f_{0}\right\|_{\mathbb{B}^{*}} \leqslant 1$. Далее, в силу плотности $\mathscr{D} \cap U_{A(M+\delta)}$ в $U_{1}$ в топологии $\tau$ сушествует $\psi \in \mathscr{D} \cap U_{A(M+\delta)}$ такая, что

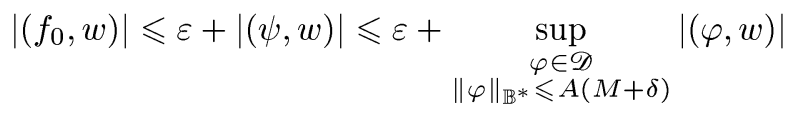

$$
\begin{aligned}
& =\varepsilon+A(M+\delta) \sup _{\substack{\varphi \in \mathscr{D} \\
\|\varphi\|_{\mathbb{B}^{*} \leqslant 1}}}|(\varphi, g)| \\
& \leqslant \varepsilon+A(M+\delta) \sup _{\substack{\varphi \in \mathscr{D} \\
\|\varphi\|_{\mathbb{B}^{*}} \leqslant 1}}|(\varphi, g-w)|+A(M+\delta) \sup _{\substack{\varphi \in \mathscr{D} \\
\|\varphi\|_{\mathbb{B}^{*} \leqslant 1}}}|(\varphi, w)| \\
& \leqslant \varepsilon+A(M+\delta) \varepsilon+A(M+\delta) \mathscr{P}[g] \leqslant \varepsilon_{1}+A(M+\delta) \mathscr{P}[g] .
\end{aligned}
$$

Здесь учтено, что $|(\varphi, g-w)| \leqslant\|\varphi\|_{\mathbb{B}^{*}}\|g-w\|_{\mathbb{B}}<\varepsilon$. Отсюда и из (6.23) получаем, что $\|g\|_{\mathbb{B}} \leqslant \varepsilon_{2}+A(M+\delta) \mathscr{P}[g]$. Так как $\varepsilon_{2}$ и $\delta$ можно считать сколь угодно мальми, лемма доказана.

Из плотности вложений $\mathscr{D} \subset \mathbb{B} \subset \mathscr{D}^{\prime}$ вытекают вложения $\mathscr{D} \subset \mathbb{B}^{*} \subset \mathscr{D}^{\prime}$, причем последнее вложение плотное. Заметим, что каноническое отображение $\mathbb{B}^{* *} \rightarrow \mathscr{D}^{\prime}$, вообще говоря, не взаимно однозначно. Обозначим через $K$ ядро этого отображения. Тогда факторпространство $\mathbb{B}^{* *} / K$ вложено в $\mathscr{D}^{\prime}$. Нетрудно показать, что

$$
\mathbb{B}^{* *} / K=\left(\overline{\mathscr{D}}^{\mathbb{B}^{*}}\right)^{*}
$$

Выясним, из каких обобщенных функций из $\mathscr{D}^{\prime}$ состоит пространство $\mathbb{B}^{* *} / K$.

ЛЕмма 6.3. Имеет место равенство

$$
\mathbb{B}^{* *} / K=\left\{g \in \mathscr{D}^{\prime}: \mathscr{P}[g]=\sup _{\substack{\varphi \in \mathscr{D} \\\|\varphi\|_{\mathbb{B}^{*} \leqslant 1}}}|(g(\xi), \varphi(\xi))|<\infty\right\}
$$

При этом

$$
\mathscr{P}[g]=\|g\|_{\mathbb{B}^{* *} / K}
$$


ДоКАЗАТЕЛЬСтво. Пусть $g \in \mathbb{B}^{* *} / K$, а $\check{g}-$ любой его представитель в $\mathbb{B}^{* *}$. Его ограничение на $\overline{\mathscr{D}}^{\mathbb{B}^{*}}$ (см. (1.19)) - тоже линейньй непрерьвньй функционал. Обозначим $\check{g}_{1}$ его продолжение на все $\mathbb{B}^{*}$, так что

$$
\left\|\check{g}_{1}\right\|_{\mathbb{B}^{* *}}=\left\|\left.\check{g}\right|_{\mathscr{D}^{\mathbb{B}^{*}}}\right\|_{\left(\overline{\mathscr{D}}^{\mathbb{B}^{*}}\right)^{*}} \cdot
$$

Такое продолжение существует в силу теоремы Хана-Банаха. Очевидно, что $\check{g}_{1}$ также является представителем $g$, ибо $\check{g}$ и $\check{g}_{1}$ совпадают на $\mathscr{D}$. Так как $\mathscr{D}$ плотно вложено в $\overline{\mathscr{D}}^{\mathbb{B}^{*}}$, имеем

$$
\begin{aligned}
\left\|\check{g}_{1}\right\|_{\mathbb{B}^{* *}}=\left\|\left.\check{g}\right|_{\overline{\mathscr{D}}^{\mathbb{B}^{*}}}\right\|_{\left(\overline{\mathscr{D}}^{\mathbb{B}^{*}}\right)^{*}}=\sup _{\substack{\varphi \in \overline{\mathscr{D}}^{\mathbb{B}^{*}} \\
\|\varphi\|_{\mathbb{B}^{*}} \leqslant 1}}|\check{g}(\varphi)| \\
=\sup _{\substack{\varphi \in \mathscr{D} \\
\|\varphi\|_{\mathbb{P}^{*} \leqslant 1}}}|\hat{g}(\varphi)|=\sup _{\substack{\varphi \in \mathscr{D} \\
\|\varphi\|_{\mathbb{B}^{*}} \leqslant 1}}|(g, \varphi)|=\mathscr{P}[g] .
\end{aligned}
$$

Отсюда следует, что $\|g\|_{\mathbb{B}^{* *} / K}=\inf _{\check{g} \in g}\|\check{g}\|_{\mathbb{B}^{* *}} \leqslant\left\|\check{g}_{1}\right\|_{\mathbb{B}^{* *}}=\mathscr{P}[g]$. Теперь из очевидного неравенства

$$
\|\check{g}\|_{\mathbb{B}^{* *}}=\sup _{\substack{\varphi \in \mathbb{B}^{*} \\\|\varphi\|_{\mathbb{B}^{*}} \leqslant 1}}|\check{g}(\varphi)| \geqslant \sup _{\substack{\varphi \in \mathscr{D} \\\|\varphi\|_{\mathbb{B}^{*}} \leqslant 1}}|(g, \varphi)|=\mathscr{P}[g],
$$

переходя к нижней грани по всем $\check{g} \in g$, получаем неравенство

$$
\|g\|_{\mathbb{B}^{* *} / K}=\inf _{\hat{g} \in g}\|\check{g}\|_{\mathbb{B}^{* *}} \geqslant \mathscr{P}[g] .
$$

Обратно, пусть $g \in \mathscr{D}^{\prime}$ и $\mathscr{P}[g]<\infty$; тогда эта обобщенная функция порождает непрерывный линейный функционал на $\overline{\mathscr{D}}^{\mathbb{B}^{*}}$. По теореме Хана-Банаха его можно продолжить на все $\mathbb{B}^{*}$, т.е. до элемента из $\mathbb{B}^{* *}$. Он порождает класс эквивалентности $g$ по ядру $K$. Это и есть прообраз $g$ при вложении $\mathbb{B}^{* *} / K$ в $\mathscr{D}^{\prime}$. Лемма доказана.

СлЕДСТВИЕ 6.2. Вложение $\mathbb{B} \subset \mathbb{B}^{* *} / K$ изометрическое (в смысле әквивалентности норм).

Утверждение вытекает из того факта, что $\mathbb{B} \subset \mathscr{D}^{\prime}$ и $\mathbb{B}^{* *} / K \subset \mathscr{D}^{\prime}$. Кроме того, $\|\cdot\|_{\mathbb{B}} \sim \mathscr{P}[\cdot]$ для элементов из $\mathbb{B}$, а $\mathbb{B}^{* *} / K$ есть множество обобщенных функций, для которых норма $\mathscr{P}[\cdot]$ конечна.

Таким образом, $\mathbb{B}$ - замкнутое подпространство $\mathbb{B}^{* *} / K$.

Обозначим через $\left(\mathbb{B}^{* *} / K\right)_{\text {fin }}$ пространство, полученное замыканием финитных обобщенных функций из $\mathbb{B}^{* *} / K$. Тогда справедливо следующее

УТВЕРЖДЕНИЕ 6.4. Имеем

$$
\left(\mathbb{B}^{* *} / K\right)_{\text {fin }} \subset \widehat{V}_{\mathbb{B}}^{J^{\infty}} \subset \mathbb{B}^{* *} / K .
$$

При әтом оба вложения изометричны (в смысле әквивалентности норм). 
ДокАЗАТЕЛЬСТво. Пусть $f$ принадлежит $\mathbb{B}^{* *} / K$ и имеет компактный носитель; тогда $L_{f}^{w}(x, y)=\left(f(\xi), \frac{1}{y} w\left(\frac{x-\xi}{y}\right)\right) \in \mathscr{D} \subset \mathbb{B}$. Здесь $w \in \mathscr{D}, \int w(\xi) d \xi=1$ и $\operatorname{supp} w \subset[-1,+1]$. Согласно лемме 6.2

$$
\begin{aligned}
\left\|L_{f}^{w}(x, y)\right\|_{\mathbb{B}} & \leqslant C \mathscr{P}\left[L_{f}^{w}(x, y)\right]=C \sup _{\substack{\varphi \in \mathscr{D} \\
\|\varphi\|_{\mathbb{B}^{*} \leqslant 1}}}\left|\int \varphi(x)\left(f(\xi), \frac{1}{y} w\left(\frac{x-\xi}{y}\right)\right) d x\right| \\
& =C \sup _{\substack{\varphi \in \mathscr{D} \\
\|\varphi\|_{\mathbb{B}^{*}} \leqslant 1}}\left|\left(f(\xi), \int \varphi(x) \frac{1}{y} w\left(\frac{x-\xi}{y}\right) d x\right)\right|
\end{aligned}
$$

Учитывая, что $\|\varphi\|_{\mathbb{B}^{*}} \leqslant 1$, имеем

$$
\begin{aligned}
& \left\|\int \varphi(x) \frac{1}{y} w\left(\frac{x-\xi}{y}\right) d x\right\|_{\mathbb{B}^{*}}=\left\|\int \varphi(\xi-x y) w(x) d x\right\|_{\mathbb{B}^{*}} \\
& \leqslant \sup _{a \in \operatorname{supp} w}\|\varphi(\cdot+a)\|_{\mathbb{B}^{*}}\left|\int w(x) d x\right| \leqslant \sup _{a \in \operatorname{supp} w} M e^{\beta|a|}\|\varphi\|_{\mathbb{B}^{*}} \leqslant M e^{\beta} .
\end{aligned}
$$

Теперь из (6.27) получаем

$$
\left\|L_{f}^{w}(x, y)\right\|_{\mathbb{B}} \leqslant C \sup _{\substack{\psi \in \mathscr{D} \\\|\psi\|_{\mathbb{B}^{*}} \leqslant M e^{\beta}}}|(f, \psi)|=C \mathscr{P}[f] .
$$

Отсюда, пользуясь леммой 6.3 , имеем

$$
\|f\|_{\widehat{V}_{\mathbb{B}}^{J \infty}}=\sup _{0<y \leqslant 1}\left\|L_{f}^{w}(x, y)\right\|_{\mathbb{B}} \leqslant C \mathscr{P}[f]=C\|f\|_{\mathbb{B}^{* *} / K} .
$$

Замыкая множество финитных функций $f \in \mathbb{B}^{* *} / K$, получаем первое из включений (6.26).

Пусть теперь $f \in \widehat{V}_{\mathbb{B}}^{\mathbb{D}^{\infty}}$. Пользуясь леммой 6.3, имеем

$$
\begin{aligned}
\|f\|_{\widehat{V}_{\mathbb{B}}^{J \infty}} & =\sup _{0<y \leqslant 1}\left\|L_{f}^{w}(x, y)\right\|_{\mathbb{B}} \geqslant \sup _{0<y \leqslant 1} \mathscr{P}\left[L_{f}^{w}(x, y)\right] \\
& =\sup _{0<y \leqslant 1} \sup _{\substack{\varphi \in \mathscr{D} \\
\|\varphi\|_{\mathbb{B}^{*}} \leqslant 1}}\left|\int \varphi(x)\left(f(\xi), \frac{1}{y} w\left(\frac{x-\xi}{y}\right)\right) d x\right| .
\end{aligned}
$$

Замечая теперь, что для $f \in \mathscr{D}^{\prime}$ справедливо соотношение

$$
\int \varphi(x)\left(f(\xi), \frac{1}{y} w\left(\frac{x-\xi}{y}\right)\right) d x \underset{y \rightarrow 0}{\longrightarrow}(f, \varphi) \int w(x) d x
$$

(напомним, что $\int w(x) d x=1$ ), из (6.29) получаем

$$
\|f\|_{\widehat{V}_{\mathbb{B}}^{J \infty}} \geqslant \sup _{\substack{\varphi \in \mathscr{D} \\\|\varphi\|_{\mathbb{B}^{*} \leqslant 1}}}|(f, \varphi)|=\mathscr{P}[f] .
$$

Снова пользуясь леммой 6.3, имеем

$$
\|f\|_{\mathbb{B}^{* *} / K}=\mathscr{P}[f] \leqslant\|f\|_{\widehat{V}_{\mathbb{B}}^{\mathbb{J}}} .
$$

Оба включения доказаны. Теперь если $f \in \widehat{V}_{\mathbb{B}}^{\mathbb{J} \infty}$, то $L_{f}^{w}(\eta, y) \in \mathbb{B}$ для $y \in$ $(0,1)$. Повторяя все выкладки, начиная с $(6.27)$, получим оценку $(6.28)$, обратную к (6.30), что доказывает изометричность второго включения в (6.26). А так как 
$\left(\mathbb{B}^{* *} / K\right)_{\text {fin }}$ изометрично вкладывается в $\mathbb{B}^{* *} / K$, то отсюда вытекает и изометричность первого включения. Утверждение доказано.

\section{СлЕДСТВИЕ 6.3. Имеем}

$$
\left(\widehat{V}_{\mathbb{B}}^{\mathrm{J}^{\infty}}\right)_{0}=\mathbb{B}
$$

причем нормы әквивалентны.

СлЕДСТВИЕ 6.4. Если $\mathbb{B}$ рефлексивно, то $\widehat{V}_{\mathbb{B}}^{\rrbracket^{\infty}}=\mathbb{B}$.

Действительно, в этом случае ядро $K$ тривиально и утверждение вытекает из включений (6.26).

СлЕДСТВИЕ 6.5. Справедливы включения $E_{\beta} \subset \mathbb{B} \subset E_{\beta}^{\prime}$, причем $E_{\beta}$ плотно вложено в $\mathbb{B}$. Здесь $\beta$ берется из (6.2).

Первое включение уже доказано (см. (6.4)), второе вытекает из цепочки доказанных вьше включений

$$
\mathbb{B}=\left(\widehat{V}_{\mathbb{B}}^{\mathbb{J}^{\infty}}\right)_{0} \subset \widehat{V}_{\mathbb{B}}^{\mathbb{J}^{\infty}} \subset V_{\mathbb{B}}^{\mathbb{J} \infty} \subset E_{\beta}^{\prime} .
$$

Последнее включение следует из леммы 6.1.

СлЕДСТвИЕ 6.6. Если автомодельный функиионал ل имеет порядок $\alpha>0$, $m o \mathbb{B} \subset V_{\mathbb{B}}^{\mathbb{J}}$.

Это непосредственно следует из того факта, что

$$
\mathbb{J}(\eta(k)) \leqslant C_{\varepsilon} \mathbb{J}^{\infty}\left(\frac{1}{k^{\alpha-\varepsilon}} \eta(k)\right) \leqslant C_{\varepsilon} \mathbb{J}^{\infty}(\eta(k)),
$$

тем самым $V_{\mathbb{B}}^{\mathbb{J}^{\infty}} \subset V_{\mathbb{B}}^{\mathbb{J}}$, и осталось только заметить, что $\mathbb{B} \subset V_{\mathbb{B}}^{\mathbb{J}^{\infty}}$.

ЗАмЕЧАниЕ 6.5. Если группа сдвигов в $\mathbb{B}$ удовлетворяет условию (6.19), то

$$
\mathscr{S} \subset \mathbb{B} \subset \mathscr{S}^{\prime}
$$

причем $\mathscr{S}$ плотно вложено в $\mathbb{B}$. Это следует из следствия 6.5 при $\beta=0$.

Пусть фиксированы банахово пространство $\mathbb{B}$ и автомодельный функционал $\mathbb{J}$. Покажем далее, что пространства $V_{\mathbb{B}}^{\mathbb{J}\left(\frac{1}{\rho} \cdot\right)}$ изоморфны для любой автомодельной функции $\rho(k)$. Изоморфизм осушествляется некоторым псевдодифференциальным оператором. Для этого понадобятся некоторые предварительные построения.

ЛЕмма 6.4. Пусть $\rho(k)$ - автомодельная функиия порядка $\alpha$. Обобщенная функиия $f$ принадлежит $V_{\mathbb{B}}^{\mathbb{J}^{\infty}\left(\frac{1}{\rho} \cdot\right)}$ тогда и только тогда, когда

$$
f^{\prime} \in V_{\mathbb{B}}^{\mathbb{J}^{\infty}\left(\frac{1}{k \rho(k)} \cdot\right)} \quad u \quad f * \varphi_{0} \in \mathbb{B} \quad \forall \varphi_{0} \in \mathscr{D} \backslash \stackrel{0}{\mathscr{D}} .
$$


ДоказАтельство. Необходимость. Справедливы соотношения

$$
\begin{aligned}
\frac{1}{k} L_{f^{\prime}}^{\varphi}\left(x, \frac{1}{k}\right) & =\frac{1}{k}\left(f^{\prime}(\xi), k \varphi(k(x-\xi))\right)=\left(f(\xi), k \varphi^{\prime}(k(x-\xi))\right)=L_{f}^{\varphi^{\prime}}\left(x, \frac{1}{k}\right) \\
L_{f^{\prime}}^{\varphi_{0}}(x, 1) & =\left(f^{\prime}(\xi), \varphi_{0}(x-\xi)\right)=\left(f(\xi), \varphi_{0}^{\prime}(x-\xi)\right)=L_{f}^{\varphi_{0}^{\prime}}(x, 1) .
\end{aligned}
$$

Пусть $\varphi \in \stackrel{n}{\mathscr{D}}$, где $n>[-\alpha]+1$. Замечая, что $\varphi^{\prime} \in \stackrel{n+1}{\mathscr{D}}$, получим

$$
\sup _{k>1} \frac{1}{k \rho(k)}\left\|L_{f^{\prime}}^{\varphi}\left(x, \frac{1}{k}\right)\right\|_{\mathbb{B}}=\sup _{k>1} \frac{1}{\rho(k)}\left\|L_{f}^{\varphi^{\prime}}\left(x, \frac{1}{k}\right)\right\|_{\mathbb{B}} \leqslant C\|f\|_{V_{\mathbb{B}}^{\mathbb{J} \infty}\left(\frac{1}{\rho} \cdot\right)} .
$$

Далее,

$$
\begin{aligned}
\left\|L_{f^{\prime}}^{\varphi_{0}}(x, 1)\right\|_{\mathbb{B}} & =\left\|L_{f}^{\varphi_{0}^{\prime}}(x, 1)\right\|_{\mathbb{B}} \leqslant c\left(\left\|L_{f}^{\varphi_{0}}(x, 1)\right\|_{\mathbb{B}}+\sup _{k>1} \frac{1}{\rho(k)}\left\|L_{f}^{\varphi^{\prime}}\left(x, \frac{1}{k}\right)\right\|_{\mathbb{B}}\right) \\
& \leqslant C\|f\|_{V_{\mathbb{B}} \|^{\mathbb{J}\left(\frac{1}{\rho} .\right)}}
\end{aligned}
$$

(см. формулу (6.8)). Отсюда следует, что если $f \in V_{\mathbb{B}}^{\text {Jळ}^{\infty}\left(\frac{1}{\rho} \cdot\right)}$, то выполнено первое включение (6.35). Второе включение тривиально.

Достаточность. Пуст
$\stackrel{n}{D}$, где $n>[-\alpha]+1$, имеем

$$
\sup _{k>1} \frac{1}{k \rho(k)}\left\|L_{f}^{\varphi^{\prime}}\left(x, \frac{1}{k}\right)\right\|_{\mathbb{B}}=\sup _{k>1} \frac{1}{\rho(k)}\left\|L_{f^{\prime}}^{\varphi}\left(x, \frac{1}{k}\right)\right\|_{\mathbb{B}} \leqslant C\left\|f^{\prime}\right\|_{V_{\mathbb{B}}^{\mathrm{J} \infty}\left(\frac{1}{\rho} \cdot\right)} .
$$

Кроме того, по условию $\left\|L_{f}^{\varphi_{0}}(x, 1)\right\|_{\mathbb{B}}=\left\|f * \varphi_{0}\right\|_{\mathbb{B}}<\infty$. Отсюда и вытекает, что $\|f\|_{V_{\mathbb{B}}^{\mathbb{J}}\left(\frac{1}{\rho} \cdot\right)}<\infty$. Лемма доказана.

ОПРЕДЕЛЕНИЕ 6.2. Пусть $\rho(k)$ - автомодельная (правильно меняюшаяся) функция. Определим множество $\mathscr{R}_{\rho}$ комплекснозначных функций $\check{\rho}(k)$, удовлетворяюших свойствам:

1) $\check{\rho}(k) \in C^{\infty}\left(\mathbb{R}^{1}\right)$;

2) $\breve{\rho}(k)$ не обрашается в нуль при $k \in \mathbb{R}^{1}$;

3) $\frac{\check{\rho}( \pm k)}{\rho(k)} \underset{k \rightarrow+\infty}{\longrightarrow}$ const $\neq 0$;

4) для любого $n=0,1, \ldots$ найдутся $c_{n}$ такие, что $\left|\check{\rho}^{(n)}( \pm k)\right| \leqslant c_{n} \frac{\rho(k)}{k^{n}}$.

Положим

$$
f_{\check{\rho}}(x)=F[\check{\rho}(k)]
$$

для любой $\check{\rho}(k)$, удовлетворяющей условиям 1$), 3)$ и 4).

Отметим, что $\check{\rho}(k)$ является мультипликатором в $\mathscr{S}$, а следовательно, $f_{\check{\rho}}$ принадлежит $\mathscr{S}^{\prime}$ и является в нем свертывателем. 
ЛЕмма 6.5. Пусть $\rho(k)$ - автомодельная функиия порядка $\alpha, C(x)=$ $M(1+|x|)^{Q}$ с любым фиксированным $M$ и целым $Q$ и $\check{\rho}(k)$ - комплекснозначная функиия, удовлетворяющая свойствам 1), 3) и 4) определения 6.2. Тогда

$$
f_{\check{\rho}}(x) \in V_{L^{1}(C(x))}^{\mathbb{J}^{\infty}\left(\frac{1}{\rho} \cdot\right)}
$$

где $L^{1}(C(x))$ - пространство $L^{1}\left(\mathbb{R}^{1}\right)$ с весом $C(x)$.

ДоказАТЕЛЬСтво. Прежде всего, заметим, что

$$
L_{f_{\check{\rho}}}^{\varphi}(x, y)=\left(f_{\check{\rho}}(\xi), \frac{1}{y} \varphi\left(\frac{x-\xi}{y}\right)\right)=\int \check{\rho}(t) \widetilde{\varphi}(y t) e^{i x t} d t
$$

где $\widetilde{\varphi}(t)$ - преобразование Фурье функции $\varphi(\xi) \in \mathscr{D}$. Так как $\check{\rho}(t) \widetilde{\varphi}(t) \in \mathscr{S}$, то $L_{f_{\tilde{\rho}}}^{\varphi_{0}}(x, 1) \in \mathscr{S}$, а потому

$$
\left\|L_{f_{\breve{\rho}}}^{\varphi_{0}}(x, 1)\right\|_{L^{1}(C(x))}=\int\left|L_{f_{\tilde{\rho}}}^{\varphi_{0}}(x, 1)\right| C(x) d x<\infty \quad \forall \varphi_{0} \in \mathscr{D} .
$$

Тем самым мы оценили второе слагаемое в норме пространства $V_{L^{1}(C(x))}^{J^{\infty}\left(\frac{1}{\rho} \cdot\right)}$ для $f_{\check{\rho}}(x)$. Теперь оценим первое слагаемое этой нормы. Выбирая функцию $\varphi(\xi) \in \mathscr{D}$ такой, чтобы все ее моменты вплоть до порядка $|Q|+2+|\alpha|$ обращались в нуль, имеем

$$
\begin{aligned}
\left|x^{N} L_{f_{\tilde{\rho}}}^{\varphi}(x, y)\right| & \leqslant\left|\int \check{\rho}(t) \widetilde{\varphi}(t y)\left(\frac{d}{d t}\right)^{N} e^{i x t} d t\right|=\left|\int e^{i x t}\left(\frac{d}{d t}\right)^{N}(\check{\rho}(t) \widetilde{\varphi}(t y)) d t\right| \\
& \leqslant \int \sum_{i+j=N} c_{i j} y^{i}\left|\check{\rho}^{(j)}\right|\left|\widetilde{\varphi}^{(i)}(y t)\right| d t .
\end{aligned}
$$

Оценим любое (типичное) слагаемое суммы в последнем интеграле. Имеем

$$
\begin{aligned}
\int y^{i}\left|\check{\rho}^{(j)}\right|\left|\widetilde{\varphi}^{(i)}(y t)\right| d t & \leqslant y^{i} c_{j} \int \frac{\rho(t)}{|t|^{j}}\left|\widetilde{\varphi}^{(i)}(y t)\right| d t \\
& \leqslant C_{1} y^{i+j-1} \rho\left(\frac{1}{y}\right) \int \frac{\rho\left(\frac{t}{y}\right)}{\rho\left(\frac{1}{y}\right)}\left|\frac{\widetilde{\varphi}^{(i)}(t)}{t^{j}}\right| d t \leqslant C_{2} y^{N-1} \rho\left(\frac{1}{y}\right) .
\end{aligned}
$$

Мы воспользовались свойством 4), оценками (1.17), (1.18) при условии, что $N \leqslant$ $|Q|+2$, и учли, что $\widetilde{\varphi}^{(i)}(t)$ имеет в нуле нуль, по крайней мере, порядка $|Q|+3+$ $|\alpha|-i$. Из полученных оценок выводим, что

$$
\left|x^{N} L_{f_{\breve{\rho}}}^{\varphi}(x, y)\right| \leqslant C_{3} y^{N-1} \rho\left(\frac{1}{y}\right), \quad 0<y \leqslant 1, \quad N \leqslant|Q|+2 .
$$


Далее имеем

$$
\begin{aligned}
\left\|L_{f_{\tilde{\rho}}}^{\varphi}(x, y)\right\|_{L^{1}(C(x))} & =\int\left|L_{f_{\tilde{\rho}}}^{\varphi}(x, y)\right| C(x) d x \\
& =\left\{\int_{|x|<y}+\int_{y<|x|<1}+\int_{|x|>1}\right\}\left|L_{f_{\tilde{\rho}}}^{\varphi}(x, y)\right| C(x) d x .
\end{aligned}
$$

Оценим эти три интеграла. Имеем

$$
\int_{|x|<y}\left|L_{f_{\breve{\rho}}}^{\varphi}(x, y)\right| C(x) d x \leqslant C_{4} \frac{1}{y} \rho\left(\frac{1}{y}\right) \int_{-y}^{y} d x \leqslant C_{5} \rho\left(\frac{1}{y}\right) .
$$

Здесь мы воспользовались оценкой (6.39) с $N=0$. Далее,

$$
\begin{aligned}
& \int_{y<|x|<1}\left|L_{f_{\tilde{\rho}}}^{\varphi}(x, y)\right| C(x) d x \leqslant \int_{y<|x|<1}\left|x^{2} L_{f_{\tilde{\rho}}}^{\varphi}(x, y)\right| \frac{C(x)}{x^{2}} d x \\
& \leqslant C_{6} y \rho\left(\frac{1}{y}\right) \int_{y<|x|<1} \frac{d x}{x^{2}} \leqslant C_{7} y \rho\left(\frac{1}{y}\right)\left[\frac{1}{y}-1\right] \leqslant C_{8} \rho\left(\frac{1}{y}\right) .
\end{aligned}
$$

Здесь использована оценка (6.39) с $N=2$. Далее,

$$
\begin{aligned}
\int_{|x|>1}\left|L_{f_{\tilde{\rho}}}^{\varphi}(x, y)\right| C(x) d x & =\int_{|x|>1}\left|x^{|Q|+2} L_{f_{\tilde{\rho}}}^{\varphi}(x, y)\right| \frac{C(x)}{x^{|Q|+2}} d x \\
\leqslant C_{9} y^{|Q|+1} \rho\left(\frac{1}{y}\right) & \leqslant C_{10} \rho\left(\frac{1}{y}\right) .
\end{aligned}
$$

Здесь также использована оценка (6.39) с $N=|Q|+2$. Эти оценки показывают, что

$$
\sup _{0<y \leqslant 1} \frac{1}{\rho\left(\frac{1}{y}\right)}\left\|L_{f_{\tilde{\rho}}}^{\varphi}(x, y)\right\|_{L^{1}(C(x))} \leqslant C_{11} .
$$

Из (6.38) и (6.40) следует утверждение леммы.

ОПРЕДЕЛЕНИЕ 6.3. Пусть $\rho(k)$ - автомодельная функция и $\beta>0$. Через $Z_{\beta}^{\rho}$ обозначаем класс аналитических в $\Pi_{-\beta}^{\beta}$ функций $\check{\rho}(\zeta)$, удовлетворяющих условиям:

1) $\check{\rho}(\zeta) \in A\left(\bar{\Pi}_{-\beta}^{\beta}\right)(\mathrm{cm} . \S 1)$;

2) $\check{\rho}(\zeta) \neq 0$ для $\forall \zeta=t+i \tau \in \bar{\Pi}_{-\beta}^{\beta}$;

3) $\frac{\check{\rho}(\zeta)}{\rho(|\zeta|)} \longrightarrow$ const $\neq 0$ при $|\zeta| \rightarrow+\infty, \quad \zeta \in \bar{\Pi}_{-\beta}^{\beta}$;

4) для любого $n=0,1, \ldots$ найдутся $c_{n}$ и $k_{0}$ такие, что $\left|\check{\rho}^{(n)}(\zeta)\right| \leqslant c_{n} \frac{1}{\left.\left.\right|_{\zeta}\right|^{n}} \rho(|\zeta|)$ при $|\zeta|>k_{0}$ и $\zeta \in \bar{\Pi}_{-\beta}^{\beta}$.

Множество преобразований Фурье функций из класса $Z_{\beta}^{\rho}$ будем обозначать $\widetilde{Z}_{\beta}^{\rho}$, т.e.

$$
\widetilde{Z}_{\beta}^{\rho}=\left\{f_{\check{\rho}}(x)=F[\check{\rho}(t)] ; \check{\rho}(t) \in Z_{\beta}^{\rho}\right\}
$$

Отметим, что $\check{\rho}$ является мультипликатором в $\widetilde{E}_{\beta}$, а $f_{\check{\rho}}$ является свертьвателем в $E_{\beta}^{\prime}$. Отметим также, что если $\check{\rho}(\zeta) \in Z_{\beta}^{\rho}$, то $\check{\rho}(\zeta \pm i \beta) \in \mathscr{R} \rho$.

Нетрудно показать, что для любой автомодельной функции $\rho(k)$ порядка $\alpha$ при любом $\beta>0$ класс $Z_{\beta}^{\rho}$ не пуст. 
ЛЕмма 6.6. Пусть $g \in V_{\mathbb{B}}^{\mathbb{J}^{\infty}\left(\frac{1}{\rho} \cdot\right)}$, где $\mathbb{B}$ - банахово пространство, удовлетворяющее аксиомам I-III, и $\rho(k)$ - автомодельная функиия порядка $\alpha>0$. Тогда для любого а и любого ограниченного в $\mathscr{D}_{a}$ семейства $\left\{\omega^{y}(\xi) \in \mathscr{D}_{a}\right.$, $0<y \leqslant 1\}$

$$
\frac{1}{\rho\left(\frac{1}{y}\right)}\left\|L_{g}^{\omega^{y}}(x, y)\right\|_{\mathbb{B}}<\text { const, } \quad 0<y \leqslant 1
$$

ДоКАЗАТЕЛЬСТВо. Из соотношения (6.7) вытекает, что

$$
\sup _{0<y \leqslant 1} \frac{1}{\rho\left(\frac{1}{y}\right)}\left\|L_{g}^{\omega}(x, y)\right\|_{\mathbb{B}} \leqslant C \mathscr{P}_{N}[\omega] \quad \forall \omega \in \mathscr{D}_{a}
$$

с некоторыми $C$ и $N$. Мы воспользовались тем, что $m<0$ при $\alpha>0$, а стало быть, $\stackrel{m}{\mathscr{D}}_{a}=\mathscr{D}_{a}$. Отсюда

$$
\frac{1}{\rho\left(\frac{1}{y}\right)}\left\|L_{g}^{\omega^{y}}(x, y)\right\|_{\mathbb{B}} \leqslant C \mathscr{P}_{N}\left[\omega^{y}\right]<\text { const. }
$$

Лемма доказана.

ЛЕмма 6.7. Пусть $\rho(k)$ - автомодельная функция порядка $\alpha, C(x)=$ $M(1+|x|)^{Q} e^{\beta|x|}$ с $M>0$ и любым цельм $Q$ и $\check{\rho}(\zeta)$ удовлетворяет условиям 1), 3) и 4) определения 6.3. Тогда

$$
f_{\check{\rho}}(x) \in V_{L^{1}(C(x))}^{\mathbb{J}^{\infty}\left(\frac{1}{\rho} \cdot\right)} .
$$

ДокАЗАТЕльСтво. Пусть $\alpha>0$. Для $\varphi \in \mathscr{D} \backslash \stackrel{0}{\mathscr{D}}$ согласно утверждению 6.3 имеем

$$
\begin{aligned}
& \left\|f_{\breve{\rho}}(x)\right\|_{\substack{V_{L^{1}(C(x))}\left(\frac{1}{\rho} \cdot\right) \\
\text { (n) }}}=\left[\sup _{0<y \leqslant 1} \frac{1}{\rho\left(\frac{1}{y}\right)} \int\left|\left(f_{\breve{\rho}}(\xi), \frac{1}{y} \varphi\left(\frac{x-\xi}{y}\right)\right)\right| e^{\beta|x|} M(1+|x|)^{Q} d x\right. \\
& \left.+\int\left|\left(f_{\check{\rho}}(\xi), \varphi(x-\xi)\right)\right| e^{\beta|x|} M(1+|x|)^{Q} d x\right] \\
& \leqslant C \max _{ \pm} \sup _{0<y \leqslant 1} \frac{1}{\rho\left(\frac{1}{y}\right)} \int\left|\left(f_{\check{\rho}}(\xi), \frac{1}{y} \varphi\left(\frac{x-\xi}{y}\right)\right)\right| e^{ \pm \beta x} M(1+|x|)^{Q} d x
\end{aligned}
$$

Оценим выражение, стоящее справа в (6.43), для знака + (для знака - оно оценивается аналогично). Имеем

$$
\begin{aligned}
\sup _{0<y \leqslant 1} & \frac{1}{\rho\left(\frac{1}{y}\right)} \int\left|\left(f_{\breve{\rho}}(\xi), \frac{1}{y} \varphi\left(\frac{x-\xi}{y}\right)\right)\right| e^{\beta x} M(1+|x|)^{Q} d x \\
& =\sup _{0<y \leqslant 1} \frac{1}{\rho\left(\frac{1}{y}\right)} \int\left|\left(e^{\beta \xi} f_{\breve{\rho}}(\xi), \frac{1}{y} e^{y \beta \frac{x-\xi}{y}} \varphi\left(\frac{x-\xi}{y}\right)\right)\right| M(1+|x|)^{Q} d x \\
& =\sup _{0<y \leqslant 1} \frac{1}{\rho\left(\frac{1}{y}\right)} \int\left|\left(f_{\check{\rho}(\cdot-i \beta)}(\xi), \frac{1}{y} \omega^{y}\left(\frac{x-\xi}{y}\right)\right)\right| M(1+|x|)^{Q} d x \\
& =\hat{\mathbb{J}}^{\infty}\left(\frac{1}{\rho\left(\frac{1}{y}\right)}\left\|L_{f_{\breve{\rho}(-i \beta)}}^{\omega^{y}}(x, y)\right\|_{L^{1}\left(M(1+|x|)^{Q}\right)}\right),
\end{aligned}
$$


где $\omega^{y}(\xi)=e^{y \beta \xi} \varphi(\xi)$. Так как $\check{\rho}(\zeta \mp i \beta)$ удовлетворяет условиям 1$\left.), 3\right)$ и 4$)$ определения 6.2 , то, согласно лемме 6.5

$$
f_{\check{\rho}(\cdot-i \beta)}(x) \in V_{L^{1}\left(M(1+|x|)^{Q}\right)}^{\mathbb{J}^{\infty}\left(\frac{1}{\rho} \cdot\right)} .
$$

Учитывая, что семейство функций $\left\{\omega^{y}(\xi): 0<y \leqslant 1\right\}$ ограничено в $\mathscr{D}_{a}(a$ таково, что $\operatorname{supp} \varphi \subset[-a, a])$, и пользуясь леммой 6.6 , из (6.44) выводим (6.42).

Пусть теперь $\alpha \leqslant 0$. Подберем $n$ таким, чтобы $n+\alpha>0$. Заметим, что $f_{\check{\rho}}^{(n)}(x)=f_{(i \zeta)^{n} \check{\rho}(\zeta)}(x)$ и $(i \zeta)^{n} \check{\rho}(\zeta)$ удовлетворяет условиям 1$\left.), 3\right)$ и 4$)$ определения 6.3 для автомодельной функции $k^{n} \rho(k)$, а ее порядок равен $n+\alpha>0$. По уже доказанному

$$
f_{(i \zeta)^{n} \check{\rho}(\zeta)} \in V_{L^{1}(C(x))}^{\mathbb{J}^{\infty}\left(\frac{1}{k^{n} \rho(k)} \cdot\right)} .
$$

Пусть теперь $\varphi_{0} \in \mathscr{D}, \int \varphi_{0}(\xi) d \xi \neq 0$; тогда

$$
\begin{aligned}
\left\|f_{\check{\rho}} * \varphi_{0}\right\|_{L^{1}(C(x))} & =\int\left|\left(f_{\check{\rho}}(\xi), \varphi_{0}(x-\xi)\right)\right| e^{\beta|x|} M(1+|x|)^{Q} d x \\
& \leqslant C \max _{ \pm} \int\left|\left(f_{\check{\rho}}(\xi), \varphi_{0}(x-\xi)\right)\right| e^{ \pm \beta x} M(1+|x|)^{Q} d x
\end{aligned}
$$

Оценим выражение, стоящее справа в (6.46), для знака + (для знака - оно оценивается аналогично). Имеем

$$
\begin{aligned}
& \int\left|\left(f_{\breve{\rho}}(\xi), \varphi_{0}(x-\xi)\right)\right| e^{\beta x} M(1+|x|)^{Q} d x \\
& \quad=\int\left|\left(e^{\beta \xi} f_{\breve{\rho}}(\xi), e^{\beta(x-\xi)} \varphi_{0}(x-\xi)\right)\right| M(1+|x|)^{Q} d x \\
& \quad=\int\left|\left(f_{\breve{\rho}(\cdot-i \beta)}(\xi), \omega(x-\xi)\right)\right| M(1+|x|)^{Q} d x \leqslant\left\|f_{\breve{\rho}(\cdot-i \beta)}(x)\right\|_{V_{L^{1}\left(M(1+|x|)^{Q}\right)}}<\infty .
\end{aligned}
$$

Здесь $\omega(\xi)=e^{\beta \xi} \varphi_{0}(\xi)$, а для последнего неравенства мы воспользовались (6.45). Таким образом, $f_{\check{\rho}} * \varphi_{0} \in L^{1}(C(x))$. Применяя теперь $n$ раз лемму 6.4 , получим $(6.42)$ и в этом случае. Лемма доказана.

УТВЕРЖДЕНИЕ 6.5. Пусть $\mathbb{B}$ - банахово пространство, в котором выполнены аксиомы I-III, с группой сдвигов $\left\{T_{a}: a \in \mathbb{R}^{1}\right\}$, удовлетворяющей условию

$$
\left\|T_{a}\right\| \leqslant M(1+|a|)^{Q} e^{\beta|a|}
$$

с некоторыми $M, Q$ и $\beta, \mathbb{J}$ - автомодельный функиионал и $\rho(k)$ - автомодельная функиия. Тогда для любой $\check{\rho}(\zeta) \in Z_{\beta}^{\rho}$ отобрахение

$$
f_{\check{\rho}^{*} *}: V_{\mathbb{B}}^{\mathbb{I}(\cdot)} \mapsto V_{\mathbb{B}}^{\mathbb{J}\left(\frac{1}{\rho} \cdot\right)}
$$

непрерывно. Здесь $f_{\check{\rho}}(x)$ - преобразование Фурье функиии $\check{\rho}$, а $f_{\check{\rho}} *-$ оператор свертки с ядром $f_{\check{\rho}}$. 
ДокАЗАТЕльСтво. Пусть $f \in V_{\mathbb{B}}^{\mathbb{J}}$. Так как $V_{\mathbb{B}}^{\mathbb{J}} \subset E_{\beta}^{\prime}$, а $f_{\check{\rho}}$-свертыватель в $E_{\beta}^{\prime}$, то свертка $f_{\check{\rho}} * f$ принадлежит $E_{\beta}^{\prime}$. Оценим ее норму в $V_{\mathbb{B}}^{\mathbb{J}\left(\frac{1}{\rho} \cdot\right)}$. Имеем

$$
\left\|f_{\breve{\rho}} * f\right\|_{V_{\mathbb{B}}^{\mathbb{J}}\left(\frac{1}{\rho} \cdot\right)}=\hat{\mathbb{J}}\left(\frac{1}{\rho\left(\frac{1}{y}\right)}\left\|L_{f_{\breve{\rho}} * f}^{\varphi}(x, y)\right\|_{\mathbb{B}}\right)+\left\|L_{f_{\tilde{\rho}} * f}^{\varphi_{0}}(x, 1)\right\|_{\mathbb{B}} .
$$

Положим $\varphi=\omega^{1} * \omega^{2}$, где $\omega^{j}, j=1,2$, из $\mathscr{D}$ и имеют достаточно большое число моментов, равных нулю. Положим также $\varphi_{0}=\omega_{0}^{1} * \omega_{0}^{2}$, где $\omega_{0}^{j}$ из $\mathscr{D} \backslash \stackrel{0}{\mathscr{D}}$. Далее нам понадобится следующая

ЛЕмма 6.8. Пусть в условиях утверждения $6.5 \quad h \in \mathbb{B}$, a $g \in L^{1}(C(x))$; mогдa $\|h * g\|_{\mathbb{B}} \leqslant\|g\|_{L^{1}(C(x))}\|h\|_{\mathbb{B}}$.

Действительно, понимая $\int h(x-a) g(a) d a$ как интеграл Фреше в банаховом пространстве $\mathbb{B}$, имеем

$$
\begin{aligned}
\left\|\int h(x-a) g(a) d a\right\|_{\mathbb{B}} & \leqslant \int|g(a)|\|h(x-a)\|_{\mathbb{B}} d a \leqslant \int C(a)|g(a)| d a\|h\|_{\mathbb{B}} \\
& =\|g\|_{L^{1}(C(x))}\|h\|_{\mathbb{B}},
\end{aligned}
$$

что и требовалось показать.

Продолжим доказательство утверждения. Пользуясь леммой 6.8 , получим

$$
\left\|L_{f_{\tilde{\rho}} * \omega^{2}}^{\omega^{1} * \omega^{2}}(x, y)\right\|_{\mathbb{B}}=\left\|L_{f_{\tilde{\rho}}}^{\omega^{1}}(x, y) * L_{f}^{\omega^{2}}(x, y)\right\|_{\mathbb{B}} \leqslant\left\|L_{f}^{\omega^{2}}(x, y)\right\|_{\mathbb{B}}\left\|L_{f_{\tilde{\rho}}}^{\omega^{1}}(x, y)\right\|_{L^{1}(C(x))} .
$$

Отсюда имеем

$$
\begin{aligned}
& \hat{\mathbb{J}}\left(\frac{1}{\rho\left(\frac{1}{y}\right)}\left\|L_{f_{\tilde{\rho}} * f}^{\omega^{1} * \omega^{2}}(x, y)\right\|_{\mathbb{B}}\right) \leqslant \hat{\mathbb{J}}\left(\frac{1}{\rho\left(\frac{1}{y}\right)}\left\|L_{f}^{\omega^{2}}(x, y)\right\|_{\mathbb{B}}\left\|L_{f_{\tilde{\rho}}}^{\omega^{1}}(x, y)\right\|_{L^{1}(C(x))}\right) \\
& \leqslant \sup _{0<y \leqslant 1} \frac{1}{\rho\left(\frac{1}{y}\right)}\left\|L_{f_{\breve{\rho}}}^{\omega^{1}}(x, y)\right\|_{L^{1}(C(x))} \hat{\mathbb{J}}\left(\left\|L_{f}^{\omega^{2}}(x, y)\right\|_{\mathbb{B}}\right) \leqslant\left\|f_{\check{\rho}}\right\|_{V_{L^{1}(C(x))}^{\mathrm{J} \infty}\left(\frac{1}{\rho} \cdot\right)}\|f\|_{V_{\mathbb{B}}^{\mathbb{J}}} .
\end{aligned}
$$

Аналогично оценивается и второе слагаемое в (6.49). Утверждение доказано.

СлЕДСТвИЕ 6.7. В условиях утверждения 6.5 отображение (6.48) осуществляет изоморфизм пространств $V_{\mathbb{B}}^{\mathbb{J}(\cdot)} u V_{\mathbb{B}}^{\mathbb{J}\left(\frac{1}{\rho} \cdot\right)}$.

Это легко следует из того, что $\frac{1}{\tilde{\rho}} \in Z_{\beta}^{\frac{1}{\rho}}$ и отображение $f_{\frac{1}{\tilde{\rho}}} *$ является обратным к отображению $f_{\tilde{\rho}} *$. 
УТВЕРЖДЕНИЕ 6.6. Пусть $\mathbb{B}$ - банахово пространство, в котором выполненьи аксиомы I-III, с группой сдвигов $\left\{T_{a}: a \in \mathbb{R}^{1}\right\}$, удовлетворяющей условию

$$
\left\|T_{a}\right\| \leqslant M(1+|a|)^{Q}
$$

с некоторыми $M$ и $Q, \mathbb{J}(\cdot)$ - автомодельный функчионал и $\rho(k)$ - автомодельная (правильно меняющаяся) функиия. Тогда отображение

$$
f_{\check{\rho}^{*}}: V_{\mathbb{B}}^{\mathbb{J}(\cdot)} \mapsto V_{\mathbb{B}}^{\mathbb{J}\left(\frac{1}{\rho} \cdot\right)},
$$

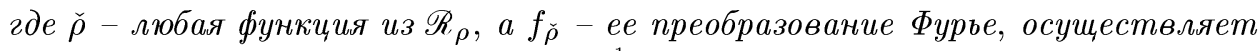
изоморфизм пространств $V_{\mathbb{B}}^{\mathbb{J}(\cdot)}$ u $V_{\mathbb{B}}^{\mathbb{J}\left(\frac{1}{\rho} \cdot\right)}$.

ДоКАЗАТЕЛЬСТВо аналогично доказательству утверждения 6.5 и следствия 6.7 , надо только учесть, что теперь $V_{\mathbb{B}}^{\mathbb{J}} \subset \mathscr{S}^{\prime}$, а $f_{\check{\rho}}$ - свертыватель в $\mathscr{S}^{\prime}$.

Как уже отмечалось (см. замечание 6.2$), \mathscr{D} \subset V_{\mathbb{B}}^{\mathbb{J}}$. Интересно выяснить, когда это вложение плотно. Этому будут посвящены несколько следующих лемм.

ЛЕмма 6.9. Пусть $\mathbb{J}-$ автомодельный функционал порядка $\alpha, \mathbb{B}-$ банахово пространство, удовлетворяющее аксиомам I-III, $u f(\xi) \in V_{\mathbb{B}}^{\mathbb{J}}$. Тогда для mого, чтобы

$$
f(\xi) \in\left(V_{\mathbb{B}}^{\mathbb{J}}\right)_{0},
$$

необходимо и достаточно, чтобы функиия $f(\xi+a)$ была непрерывна по а в пространстве $V_{\mathbb{B}}^{\mathbb{J}}$.

ДокаЗАтЕльСтво. Необходимость. Пусть $f(\xi) \in\left(V_{\mathbb{B}}^{\mathbb{J}}\right)_{0}=\overline{\mathscr{D}}_{\mathbb{\mathbb { B }}}^{V^{\mathbb{J}}}$. Тогда $\{f(\xi+$ $\left.a): a \in \mathbb{R}^{1}\right\}$ - непрерывное семейство в $V_{\mathbb{B}}^{\mathbb{J}}$, так как сдвиги непрерывны в $\mathscr{D}$, а $\mathscr{D}$ плотно и непрерывно вложено в $\left(V_{\mathbb{B}}^{\mathbb{J}}\right)_{0}$.

Достаточность. а) Рассмотрим случай $\alpha>0$. Пусть функция $f(\xi+a)$ непрерывна в $V_{\mathbb{B}}^{\mathbb{J}}$. Положим $\omega_{k}(\xi)=k \omega(k \xi)$, где $\omega(\xi)$ - "шапочка", т.е. $\omega \in \mathscr{D}$, $\int \omega(\xi) d \xi=1, \omega>0$ и $\operatorname{supp} \omega \subset[-1,+1]$. Теперь заметим, что

$$
\int f(\xi-a) \omega_{k}(a) d a \underset{k \rightarrow+\infty}{\longrightarrow} f(\xi) \text { в } V_{\mathbb{B}}^{\mathbb{J}} .
$$

(Здесь интеграл понимается как интеграл $\Phi$ реше в банаховом пространстве $V_{\mathbb{B}}^{\mathbb{J}}$.) Действительно,

$$
\begin{gathered}
\left\|\int f(\xi-a) \omega_{k}(a) d a-f(\xi)\right\|_{V_{\mathbb{B}}^{\mathbb{J}}}=\left\|\int(f(\xi-a)-f(\xi)) \omega_{k}(a) d a\right\|_{V_{\mathbb{B}}^{\mathbb{J}}} \\
\leqslant \sup _{a \in \operatorname{supp} \omega_{k}}\|f(\xi-a)-f(\xi)\|_{V_{\mathbb{B}}^{\mathbb{J}}} \int \omega_{k}(a) d a \longrightarrow 0 \text { при } k \rightarrow+\infty .
\end{gathered}
$$

Так как $\int f(\xi-a) \omega_{k}(a) d a \in \mathbb{B}$, а $\mathscr{D}$ плотно в $\mathbb{B}$, то эту свертку можно приблизить функциями из $\mathscr{D}$ в топологии $\mathbb{B}$. Поскольку $\mathbb{B} \subset V_{\mathbb{B}}^{\mathbb{J}}$ при $\alpha>0$ (см. следствие 6.6), 
то это приближение имеет место и в топологии $V_{\mathbb{B}}^{\mathbb{J}}$, что и доказывает утверждение леммы в этом случае.

b) Пусть $\alpha \leqslant 0$. Рассмотрим автомодельный функционал $\mathbb{J}_{1}(\cdot)=\mathbb{J}\left(\frac{1}{\rho} \cdot\right)$, где $\rho(k)=k^{-\alpha+\varepsilon}$. Его порядок автомодельности равен $\varepsilon>0$. Функция $f_{1}(\xi)$, равная $f_{\check{\rho}} * f$, принадлежит $V_{\mathbb{B}}^{\mathbb{J}_{1}}$, и ее сдвиги $\left\{f_{1}(\xi+a), a \in \mathbb{R}^{1}\right\}$ - непрерывное семейство в $V_{\mathbb{B}}^{\mathbb{J}_{1}}$, так как отображение $f_{\check{\rho}} *$ (согласно утверждению 6.5 и следствию 6.7) устанавливает (топологический) изоморфизм между пространствами $V_{\mathbb{B}}^{\mathbb{J}}$ и $V_{\mathbb{B}}^{\mathbb{J}_{1}}$. По уже доказанному $f_{1}$ принадлежит $\left(V_{\mathbb{B}}^{\mathbb{J}_{1}}\right)_{0}$, следовательно, приближается функциями из $\mathscr{D}$. Так как отображение $f_{\check{\rho}} *$ сохраняет $E_{\beta}$ и $\check{\rho}$ - мультипликатор в $Z_{\beta}$, а $\mathscr{D}$ плотно в $E_{\beta}$, то $f$ можно приблизить в $V_{\mathbb{B}}^{\mathbb{J}}$ функциями из $E_{\beta}$. Поскольку $\mathscr{D}$ плотно в $E_{\beta}$, то любую функцию из $E_{\beta}$ можно приблизить функциями из $\mathscr{D}$ в топологии $E_{\beta}$, но в силу непрерывности вложения $E_{\beta} \subset V_{\mathbb{B}}^{\mathbb{J}}$ (см. (6.15)) это приближение имеет место и в топологии $V_{\mathbb{B}}^{\mathbb{J}}$. Отсюда следует утверждение леммы и в этом случае. Лемма доказана.

ЛЕмма 6.10. Пусть $\mathbb{J}$ - автомодельный функиионал, удовлетворяющий теореме Лебега (см. определение 2.1), $\mathbb{B}$ - банахово пространство с аксиомами I-III. Тогда

$$
V_{\mathbb{B}}^{\mathrm{d}}=\left(V_{\mathbb{B}}^{\mathrm{d}}\right)_{0}
$$

ДоказАтельство. Пусть $f(x) \in V_{\mathbb{B}}^{\mathbb{J}}$. Рассмотрим семейство сдвигов $\{f(x+a)$ : $\left.a \in \mathbb{R}^{1}\right\}$ и докажем, что оно непрерывно при $a=0$, а стало быть, и при любом $a$ в $V_{\mathbb{B}}^{\mathbb{J}} \cdot$ Имеем

$$
\begin{aligned}
\|f(x+a)-f(x)\|_{V_{\mathbb{B}}^{\mathbb{J}}}=\hat{\mathbb{J}} & \left(\left\|L_{f(x+a)}^{\varphi}(x, y)-L_{f}^{\varphi}(x, y)\right\|_{\mathbb{B}}\right) \\
& +\left\|L_{f(x+a)}^{\varphi_{0}}(x, 1)-L_{f}^{\varphi_{0}}(x, 1)\right\|_{\mathbb{B}} \\
= & \hat{\mathbb{J}}\left(\left\|L_{f}^{\varphi_{f}}(x+a, y)-L_{f}^{\varphi_{f}}(x, y)\right\|_{\mathbb{B}}\right) \\
& +\left\|L_{f}^{\varphi_{0}}(x+a, 1)-L_{f}^{\varphi_{0}}(x, 1)\right\|_{\mathbb{B}} .
\end{aligned}
$$

Поскольку сдвиги непрерьвны в $\mathbb{B}$, второе слагаемое, стоящее справа в (6.55), стремится к нулю при $a \rightarrow+0$. По тем же соображениям при фиксированном $y \in(0,1]$

$$
\left\|L_{f}^{\varphi}(x+a, y)-L_{f}^{\varphi}(x, y)\right\|_{\mathbb{B}} \rightarrow 0 \text { при } a \rightarrow+0 .
$$

Кроме того, считая $a<1$, имеем

$$
\begin{aligned}
\left\|L_{f}^{\varphi}(x+a, y)-L_{f}^{\varphi}(x, y)\right\|_{\mathbb{B}} & \leqslant\left\|L_{f}^{\varphi}(x+a, y)\right\|_{\mathbb{B}}+\left\|L_{f}^{\varphi}(x, y)\right\|_{\mathbb{B}} \\
& \leqslant(C(a)+1)\left\|L_{f}^{\varphi}(x, y)\right\|_{\mathbb{B}} \leqslant A\left\|L_{f}^{\varphi}(x, y)\right\|_{\mathbb{B} .}
\end{aligned}
$$

Так как $\hat{\mathbb{J}}\left(\left\|L_{f}^{\varphi}(x, y)\right\|_{\mathbb{B}}\right)<\infty$, то по теореме Лебега

$$
\hat{\mathbb{J}}\left(\left\|L_{f}^{\varphi}(x+a, y)-L_{f}^{\varphi}(x, y)\right\|_{\mathbb{B}}\right) \underset{a \rightarrow 0}{\longrightarrow} 0 .
$$

Теперь по лемме $6.9 f \in\left(V_{\mathbb{B}}^{\mathbb{J}}\right)_{0}$. Лемма доказана.

Ранее мы доказали, что $\mathbb{B} \subset V_{\mathbb{B}}^{\mathbb{J}^{\infty}}$ (см. (6.32)). Покажем, что

$$
V_{\mathbb{B}}^{\mathbb{J}^{1}} \subset \mathbb{B} \subset V_{\mathbb{B}}^{\mathbb{J}^{\infty}} \text {. }
$$


ЛЕмма 6.11. Пусть $\mathbb{B}$ - банахово пространство, удовлетворяющее аксиомам I-III. Тогда $V_{\mathbb{B}}^{\mathbb{I}^{1}} \subset \mathbb{B}$.

ДокАЗАТЕЛЬСТво. Так как $\mathbb{B}=\left(\widehat{V}_{\mathbb{B}}^{\mathbb{J}^{\infty}}\right)_{0}$ (см. следствие 6.3$)$, то достаточно доказать, что $V_{\mathbb{B}}^{\mathbb{J}^{1}} \subset\left(\widehat{V}_{\mathbb{B}}^{J^{\infty}}\right)_{0}$. Воспользуемся соотношением

$$
L_{f}^{\varphi}(x, y)=\int_{y}^{1} \frac{d \eta}{\eta} L_{f}^{\varphi_{1}}(x, \eta)+L_{f}^{\varphi}(x, 1), \quad \varphi_{1}(\xi)=\frac{d}{d \xi}(\xi \varphi(\xi)) .
$$

Оно получается, если проинтегрировать по $\eta$ от $y$ до 1 легко проверяемое тождество $\frac{d}{d \eta} L_{f}^{\varphi}(x, \eta)=-\frac{1}{\eta} L_{f}^{\varphi_{1}}(x, \eta)$. Пусть теперь $f \in V_{\mathbb{B}}^{\mathbb{J}^{1}}$. Пользуясь $(6.57)$, для $\varphi$ и $\varphi_{0}$ из $\mathscr{D} \backslash \mathscr{D}$ получаем

$$
\begin{aligned}
\|f\|_{\widehat{V}_{\mathbb{B}}^{J \infty}} & =\sup _{0<y \leqslant 1}\left\|L_{f}^{\varphi}(x, y)\right\|_{\mathbb{B}}+\left\|L_{f}^{\varphi_{0}}(x, 1)\right\|_{\mathbb{B}} \\
& =\sup _{0<y \leqslant 1}\left\|L_{f}^{\varphi}(x, 1)+\int_{y}^{1} \frac{1}{\eta} L_{f}^{\varphi_{1}}(x, \eta) d \eta\right\|_{\mathbb{B}}+\left\|L_{f}^{\varphi_{0}}(x, 1)\right\|_{\mathbb{B}} \\
& \leqslant\left[\left\|L_{f}^{\varphi}(x, 1)\right\|_{\mathbb{B}}+\left\|L_{f}^{\varphi_{0}}(x, 1)\right\|_{\mathbb{B}}\right]+\sup _{0<y \leqslant 1} \int_{y}^{1} \frac{d \eta}{\eta}\left\|L_{f}^{\varphi_{1}}(x, \eta)\right\|_{\mathbb{B}} \leqslant c\|f\|_{V_{\mathbb{B}}^{\mathbb{J} 1}} .
\end{aligned}
$$

(В последнем неравенстве учтено, что $\int \varphi_{1}(\xi) d \xi=0$.) Отсюда вытекает, что $V_{\mathbb{B}}^{\mathbb{J}^{1}}$ непрерывно вложено в $\widehat{V}_{\mathbb{B}}^{\mathbb{J}^{\infty}}$, а потому $\left(V_{\mathbb{B}}^{\mathbb{J}^{1}}\right)_{0} \subset\left(\widehat{V}_{\mathbb{B}}^{J^{\infty}}\right)_{0}$. По лемме $6.10\left(V_{\mathbb{B}}^{\mathbb{J}^{1}}\right)_{0}=$ $V_{\mathbb{B}}^{\mathbb{J}^{1}}$, так как $\mathbb{J}^{1}$ удовлетворяет теореме Лебега. Отсюда и следует (6.56).Лемма доказана.

СлЕДСТВИЕ 6.8. Пусть в условиях леммы 6.11 J - автомодельный функиионал порядка $\alpha<0 ;$ тогда

$$
V_{\mathbb{B}}^{\mathbb{J}} \subset \mathbb{B} .
$$

Действительно, так как $\alpha+\varepsilon<0$ при достаточно малом $\varepsilon$, то $\mathbb{J}^{1}(\psi(k)) \leqslant$ $\mathbb{J}^{1}\left(\frac{1}{k^{\alpha+\varepsilon}} \psi(k)\right) \leqslant C_{\varepsilon} \mathbb{J}(\psi(k))$ (см. (2.9)). Отсюда и из (6.56) имеем

$$
V_{\mathbb{B}}^{\mathbb{J}} \subset V_{\mathbb{B}}^{\mathbb{J}^{1}\left(\frac{1}{k^{\alpha+\varepsilon}} \cdot\right)} \subset V_{\mathbb{B}}^{\mathbb{J}^{1}} \subset \mathbb{B} .
$$

Покажем, что введенные банаховы пространства $V_{\mathbb{B}}^{\mathbb{J}}$ при $\alpha<0$ допускают другое, более традиционное, описание и в частных случаях совпадают с широко известными и часто встречающимися на практике пространствами типа Бесова-Никольского.

УТВЕРЖДЕНИЕ 6.7. Пусть $\mathbb{J}-$ автомодельный функиионал порядка $\alpha,-1<$ $\alpha<0$, и банахово пространство $\mathbb{B}$ удовлетворяет аксиомам I-III. Тогда

$$
V_{\mathbb{B}}^{\mathbb{J}}=\left\{f(x) \in \mathbb{B}: \quad\|f\|_{\Lambda}=\hat{\mathbb{J}}\left(\|f(x+y)-f(x)\|_{\mathbb{B}}\right)+\|f\|_{\mathbb{B}}<\infty\right\},
$$

причем $\|\cdot\| \Lambda \sim\|f\|_{V_{\mathbb{B}}^{\mathbb{J}}}$. 
ДокаЗАтЕльство. Пусть $f \in V_{\mathbb{B}}^{\mathbb{J}}$. Докажем, что $f \in \mathbb{B}$ и $\|f\|_{\Lambda} \leqslant C\|f\|_{V_{\mathbb{B}}^{\mathbb{J}}}$. Первое включение следует из того, что $\alpha<0$, и из (6.58). Отсюда же следует, что $\|f\|_{\mathbb{B}} \leqslant C_{1}\|f\|_{V_{\mathbb{B}}^{\mathrm{J}}}$. Далее, пусть $\varphi \in \mathscr{D}, \int \varphi(x) d x=1$. Имеем

$$
\begin{aligned}
\|f(x+y)-f(x)\|_{\mathbb{B}} \leqslant & \left\|f(x+y)-L_{f}^{\varphi}(x+y, y)\right\|_{\mathbb{B}} \\
& +\left\|L_{f}^{\varphi}(x+y, y)-L_{f}^{\varphi}(x, y)\right\|_{\mathbb{B}}+\left\|L_{f}^{\varphi}(x, y)-f(x)\right\|_{\mathbb{B}} .
\end{aligned}
$$

Оценим каждое слагаемое в правой части. Рассмотрим последнее слагаемое. Так как $f \in \mathbb{B}$, то $L_{f}^{\varphi}(x, y) \rightarrow f(x)$ при $y \rightarrow 0$ в $\mathbb{B}$. Переходя к пределу в $\mathbb{B}$ при $y_{0} \rightarrow 0$ в равенстве $\int_{y_{0}}^{y} \frac{d}{d \eta} L_{f}^{\varphi}(x, \eta) d \eta=L_{f}^{\varphi}(x, y)-L_{f}^{\varphi}\left(x, y_{0}\right)$, получим

$$
L_{f}^{\varphi}(x, y)-f(x)=\int_{0}^{y} \frac{d}{d \eta} L_{f}^{\varphi}(x, \eta) d \eta .
$$

Так как $\frac{d}{d y} L_{f}^{\varphi}(x, y)=-\frac{1}{y} L_{f}^{\varphi_{1}}(x, y)$, где $\varphi_{1}(x)=(x \varphi(x))^{\prime}$, получим

$$
L_{f}^{\varphi}(x, y)-f(x)=-\int_{0}^{y} \frac{1}{\eta} L_{f}^{\varphi_{1}}(x, \eta) d \eta .
$$

Следовательно,

$$
\begin{aligned}
\hat{\mathbb{J}}\left(\left\|L_{f}^{\varphi}(x, y)-f(x)\right\|_{\mathbb{B}}\right) & \leqslant \hat{\mathbb{J}}\left(\int_{0}^{y} \frac{1}{\eta}\left\|L_{f}^{\varphi_{1}}(x, \eta)\right\|_{\mathbb{B}} d \eta\right) \\
& \leqslant C_{2} \hat{\mathbb{J}}\left(\left\|L_{f}^{\varphi_{1}}(x, \eta)\right\|_{\mathbb{B}}\right) \leqslant C_{2}\|f\|_{V_{\mathbb{B}}} .
\end{aligned}
$$

В предпоследнем неравенстве мы воспользовались неравенством Харди для $\alpha<0$, а в последнем неравенстве - тем, что $\int \varphi_{1}(x) d x=\int \frac{d}{d x}(x \varphi(x)) d x=0$.

Для первого слагаемого справа в (6.60) получим

$$
\begin{aligned}
\hat{\mathbb{J}}\left(\left\|f(x+y)-L_{f}^{\varphi}(x+y, y)\right\|_{\mathbb{B}}\right) & =\hat{\mathbb{J}}\left(\left\|T_{y}\left(f(x)-L_{f}^{\varphi}(x, y)\right)\right\|_{\mathbb{B}}\right) \\
& \leqslant C(1) \hat{\mathbb{J}}\left(\left\|f(x)-L_{f}^{\varphi}(x, y)\right\|_{\mathbb{B}}\right) \leqslant c\|f\|_{V_{\mathbb{B}}^{\mathbb{J}}} .
\end{aligned}
$$

Наконец, для среднего слагаемого в (6.60) имеем

$$
\begin{gathered}
\left\|L_{f}^{\varphi}(x+y, y)-L_{f}^{\varphi}(x, y)\right\|_{\mathbb{B}}=\left\|\left(f(\xi), \frac{1}{y}\left[\varphi\left(\frac{x+y-\xi}{y}\right)-\varphi\left(\frac{x-\xi}{y}\right)\right]\right)\right\|_{\mathbb{B}} \\
=\left\|\left(f(\xi), \frac{1}{y} \varphi_{2}\left(\frac{x-\xi}{y}\right)\right)\right\|_{\mathbb{B}}, \quad \varphi_{2}(\xi)=\varphi(\xi+1)-\varphi(\xi) .
\end{gathered}
$$

Учитывая, что $\int \varphi_{2}(x) d x=0$, получаем

$$
\hat{\mathbb{J}}\left(\left\|L_{f}^{\varphi}(x+y, y)-L_{f}^{\varphi}(x, y)\right\|_{\mathbb{B}}\right) \leqslant \hat{\mathbb{J}}\left(\left\|L_{f}^{\varphi_{2}}(x, y)\right\|_{\mathbb{B}}\right) \leqslant c\|f\|_{V_{\mathbb{B}}^{\mathbb{J}}}
$$


Суммируя полученные неравенства, выводим $\|f\|_{\Lambda} \leqslant C\|f\|_{V_{\mathbb{B}}^{\mathrm{J}}}$.

Обратно, пусть $f \in \mathbb{B}$ и $\|f\|_{\Lambda}<\infty$. Возмем $\varphi \in \mathscr{D}, \int \varphi(x) d x \neq 0$, и $\operatorname{supp} \varphi \subset$ $[-1,+1]$. Учитывая, что $\int \varphi^{\prime}(x) d x=0$, имеем

$$
\begin{aligned}
L_{f}^{\varphi^{\prime}}(x, y) & =\left(f(\xi), \frac{1}{y} \varphi^{\prime}\left(\frac{x-\xi}{y}\right)\right)=\left(f(x-y \xi), \varphi^{\prime}(\xi)\right)=\left(f(x-y \xi)-f(x), \varphi^{\prime}(\xi)\right) \\
& =\left(f(x-\xi)-f(x), \frac{1}{y} \varphi^{\prime}\left(\frac{\xi}{y}\right)\right)=\int[f(x-\xi)-f(x)] \frac{1}{y} \varphi^{\prime}\left(\frac{\xi}{y}\right) d \xi
\end{aligned}
$$

Здесь интеграл понимается как интеграл $Ф$ реше в $\mathbb{B}$ (сдвиги непрерывны в $\mathbb{B})$. Далее имеем

$$
\begin{aligned}
\left\|L_{f}^{\varphi^{\prime}}(x, y)\right\|_{\mathbb{B}} & \leqslant \int\|f(x-\xi)-f(x)\|_{\mathbb{B}} \frac{1}{y}\left|\varphi^{\prime}\left(\frac{\xi}{y}\right)\right| d \xi \\
& \leqslant \sup _{\xi}\left|\xi \varphi^{\prime}(\xi)\right| \cdot \int_{-y}^{y} \frac{d \xi}{\xi}\|f(x-\xi)-f(x)\|_{\mathbb{B}} \\
& \leqslant C_{2}\left\{\int_{-y}^{0}+\int_{0}^{y}\right\} \frac{d \xi}{\xi}\|f(x-\xi)-f(x)\|_{\mathbb{B}} .
\end{aligned}
$$

Отсюда, пользуясь неравенством Харди, получаем

$$
\begin{aligned}
& \hat{\mathbb{J}}\left(\left\|L_{f}^{\varphi^{\prime}}(x, y)\right\|_{\mathbb{B}}\right) \leqslant \\
& \quad \leqslant \hat{\mathbb{J}}\left(\int_{0}^{y} \frac{d \xi}{\xi}\|f(x-\xi)-f(x)\|_{\mathbb{B}}\right)+\hat{\mathbb{J}}\left(\int_{-y}^{0} \frac{d \xi}{\xi}\|f(x-\xi)-f(x)\|_{\mathbb{B}}\right) \\
& \quad \leqslant C\left[\hat{\mathbb{J}}\left(\|f(x-y)-f(x)\|_{\mathbb{B}}\right)+\hat{\mathbb{J}}\left(\|f(x+y)-f(x)\|_{\mathbb{B}}\right)\right] \\
& \quad \leqslant C\left[C(1) \hat{\mathbb{J}}\left(\|f(x)-f(x+y)\|_{\mathbb{B}}\right)+\hat{\mathbb{J}}\left(\|f(x+y)-f(x)\|_{\mathbb{B}}\right)\right] \leqslant C_{4}\|f\|_{\Lambda} .
\end{aligned}
$$

Отсюда и из неравенства

$$
\|(f(\xi), \varphi(x-\xi))\|_{\mathbb{B}} \leqslant c\|f\|_{\mathbb{B}} \int|\varphi(\xi)| d \xi \leqslant c_{1}\|f\|_{\Lambda}
$$

получим $\|f\|_{V_{\mathbb{B}}^{\mathbb{J}}} \leqslant c\|f\|_{\Lambda}$. Утверждение доказано.

ЗАмЕчАнИЕ 6.6. Утверждение 6.7 останется справедливым и при любом отрицательном $\alpha$, но если $-2<\alpha<0$, то вместо разности $f(x+y)-f(x)$ в $(6.59)$ нужно брать вторую разность $[f(x+y)-2 f(x)+f(x-y)]$, при $-3<\alpha<0$ - третью разность и т.д.

\section{Приложение}

Здесь мы докажем ряд вспомагательных утверждений, которые были использованы в основном тексте.

Следуюшие четыре леммы являются некоторыми вариантами классической теоремы Карлесона о короне. Мы приводим их в том виде, который нам был нужен 
в основном тексте. Идеи доказательства достаточно традиционны и содержатся, например, в [9] (см. также цитируемую там литературу).

ЛЕмма П.1 (о короне для пространства $Z$ ). Пусть $\Omega$ - произвольное множество индексов и задано семейство челых аналитических функиий $\left\{\chi_{\beta}(z)\right.$, $\beta \in \Omega\}$, удовлетворяющих следующим оченкам:

$$
\begin{gathered}
\left|\chi_{\beta}(z)\right| \leqslant A e^{a|y|}(1+|x|)^{Q}, \quad z=x+i y \in \mathbb{C}, \quad \beta \in \Omega, \\
\sup _{\beta \in \Omega}\left|\chi_{\beta}(z)\right| \geqslant \frac{c e^{-a|y|}}{(1+|z|)^{Q}}, \quad z \in \mathbb{C},
\end{gathered}
$$

для некоторых $A, a$, с и $Q$. Тогда существуют счетное множсество индексов $I \subset \Omega$, числа $\lambda_{\beta}>0, \quad \beta \in I, \quad \sum_{\beta \in I} \lambda_{\beta}=1$, и целье аналитические функции $\left\{g_{\beta}(z), \beta \in I\right\}$ maкие, что

$$
\left|g_{\beta}(z)\right| \leqslant A_{1} e^{a_{1}|y|}(1+|x|)^{Q_{1}}, \quad z \in \mathbb{C},
$$

для некоторых $A_{1}, a_{1}, Q_{1} u$

$$
\sum_{\beta \in I} \lambda_{\beta} g_{\beta}(z) \chi_{\beta}(z)=1, \quad z \in \mathbb{C}
$$

Докажем сначала следующее

УТВЕРЖДЕНИЕ П.1. Пусть $\Omega$ - произвольное множество индексов и задано семейство чельх аналитических функций $\left\{\chi_{\beta}(z), z \in \mathbb{C}, \beta \in \Omega\right\}$, удовлетворяющих оченкам (П.1) и (П.2). Тогда существуют счетное множество $I \subset \Omega$, числа $c_{2}, a_{2}, Q_{2}$ u $\lambda_{\beta}>0, \beta \in I, \sum_{\beta \in I} \lambda_{\beta}<\infty$, такие, что

$$
\sum_{\beta \in I} \lambda_{\beta}\left|\chi_{\beta}(z)\right| \geqslant \frac{c_{2} e^{-a_{2}|y|}}{(1+|x|)^{Q_{2}}}, \quad z=x+i y \in \mathbb{C} .
$$

ДокАЗАТЕЛЬСТво. Если $\Omega$ - конечное множество, то утверждение тривиально. Пусть $\Omega$ - бесконечное множество. Положим

$$
r(\beta, z)=\min \left\{1, \frac{d}{(2+|x|)^{Q}} e^{-a|y|}\left|\chi_{\beta}(z)\right|\right\}
$$

где $d$ - некоторая положительная постоянная. Покажем, что ее можно подобрать так, чтобы

$$
\left|\chi_{\beta}(\zeta)\right| \geqslant \frac{1}{2}\left|\chi_{\beta}(z)\right|, \quad|\zeta-z|<r(\beta, z)
$$


Действительно, учитывая, что при некотором $M$ вьполнено неравенство (легко вытекаюшее из формулы Коши) $\left|\chi_{\beta}^{\prime}(z)\right| \leqslant M e^{a|y|}(1+|x|)^{Q}$, имеем

$$
\begin{aligned}
\left|\chi_{\beta}(\zeta)\right| & =\left|\chi_{\beta}(z)+\int_{z}^{\zeta} \chi_{\beta}^{\prime}(\tau) d \tau\right| \geqslant\left|\chi_{\beta}(z)\right|-\left|\int_{z}^{\zeta}\right| \chi_{\beta}^{\prime}(\tau)|d| \tau|| \\
& \geqslant\left|\chi_{\beta}(z)\right|-M\left|\int_{z}^{\zeta} e^{a|\operatorname{Im} \tau|}\left(1+|\operatorname{Re} \tau|^{Q}\right) d\right| \tau|| \\
& \geqslant\left|\chi_{\beta}(z)\right|-M e^{a(|y|+1)}(2+|x|)^{Q}|\zeta-z| .
\end{aligned}
$$

Здесь интегрируем по прямой, соединяюшей точки $\zeta$ и $z$. Ясно, что (П.7) будет выполнено, если в (П.6) положить $d$, например, равным $\frac{1}{2 M e^{a}}$.

В силу (П.2) для каждой точки $z \in \mathbb{C}$ найдется $\beta_{z} \in \Omega$ такое, что

$$
\left|\chi_{\beta_{z}}(z)\right| \geqslant \frac{c}{2} e^{-a|y|} \frac{1}{(1+|x|)^{Q}}
$$

Покажем, что сушествует $d_{1}$ такое, что

$$
\left|\chi_{\beta_{z}}(\zeta)\right| \geqslant \frac{c}{4} e^{-a|y|} \frac{1}{(1+|x|)^{Q}}, \quad|\zeta-z| \leqslant d_{1} e^{-2 a|y|} \frac{1}{(2+|x|)^{2 Q}} .
$$

Действительно, при достаточно малом $d_{1}$

$$
\begin{aligned}
d_{1} e^{-2 a|y|} \frac{1}{(2+|x|)^{2 Q}} & \leqslant \min \left\{1, \frac{d}{(2+|x|)^{Q}} e^{-a|y|} \frac{c}{2} e^{-a|y|} \frac{1}{(1+|x|)^{Q}}\right\} \\
& \leqslant \min \left\{1, \frac{d}{(2+|x|)^{Q}} e^{-a|y|}\left|\chi_{\beta_{z}}(z)\right|\right\}=r(\beta, z),
\end{aligned}
$$

а потому $\left|\chi_{\beta_{z}}(\zeta)\right| \geqslant \frac{1}{2}\left|\chi_{\beta_{z}}(z)\right| \geqslant \frac{c}{4} e^{-a|y|} \frac{1}{(1+|x|)^{Q}}$.

Всю комплексную плоскость $\mathbb{C}$ разобьем на квадратики

$$
W_{n, m}=\{z \in \mathbb{C}: n-1 \leqslant x<n, \quad m-1 \leqslant y<m\}, \quad n, m \in \mathbb{Z} .
$$

Каждый квадратик $W_{n, m}$ окажется покрытым кружками с центрами в точках $z \in W_{n, m}$ радиуса $d_{1} e^{-2 a|y|} \frac{1}{(2+|x|)^{2 Q}}$. Из этого покрытия можно выбрать конечно кратное покрытие, причем его кратность не зависит от $n$ и $m$ (см. [5, с. 33]). Таким образом, каждый $W_{n, m}$ оказался покрытым не более чем $N_{n, m}=\frac{\text { const }}{\pi r_{\min }^{2}}$ кружками, где

$$
r_{\min }(n, m) \geqslant d_{1} e^{-2 a(|m|+1)} \frac{1}{(2+|n|+1)^{2 Q}} \geqslant \frac{d_{2}}{(1+|n|)^{2 Q}} e^{-2 a|m|} .
$$

Пусть $z_{i}, i=1,2, \ldots,-$ центры всех этих кружков, покрывающих все $W_{n, m}$ (а стало быть, и все $\mathbb{C}$ ) и $I=\left\{\beta_{z_{i}}, i=1,2, \ldots\right\}$ (см. (П.8)). Положим $\lambda_{i}=$ 
$\frac{1}{n^{2} m^{2} N_{n, m}}$, если кружок с центром $z_{i}$ является одним из кружков, покрывающих $W_{n, m}$. Проверим, что $I$ и $\lambda_{i}$ удовлетворяют условиям утверждения. Имеем

$$
\sum_{i \in I} \lambda_{i} \leqslant \sum_{n, m} \frac{1}{m^{2} n^{2} N_{n, m}} \cdot N_{n, m}=\sum_{n, m} \frac{1}{m^{2} n^{2}}<\infty .
$$

Проверим оценку (П.5). Пусть точка $z \in \mathbb{C}$ попадет в некоторый квадратик $W_{n_{0}, m_{0}}$, а следовательно, в некоторый кружок с центром $z_{i_{0}}$ (один из кружков его покрывающих). Имеем

$$
\begin{aligned}
& \sum_{i \in I} \lambda_{i}\left|\chi_{\beta_{z_{i}}}(z)\right| \geqslant \lambda_{i_{0}}\left|\chi_{\beta_{z_{i}}}(z)\right| \geqslant \frac{1}{n_{0}^{2} m_{0}^{2} N_{n_{0}, m_{0}}} \frac{c}{4} e^{-a\left|y_{i_{0}}\right|} \frac{1}{\left(1+\left|x_{i_{0}}\right|\right)^{Q}} \\
& \geqslant \frac{C_{1}}{n_{0}^{2} m_{0}^{2}} \frac{d_{2}^{2} e^{-4 a\left|m_{0}\right|}}{\left(1+\left|n_{0}\right|\right)^{4 Q}} \frac{c}{4} \frac{e^{-a\left|y_{i_{0}}\right|}}{\left(1+\left|x_{i_{0}}\right|\right)^{Q}} \geqslant \frac{C_{2} e^{-a_{2}|y|}}{(1+|x|)^{Q_{2}}},
\end{aligned}
$$

где $C_{1}, C_{2}$ - некоторые постоянные. Здесь мы учли, что $|y|-1 \leqslant\left|m_{0}\right| \leqslant|y|+1$, $|x|-1 \leqslant\left|n_{0}\right| \leqslant|x|+1,|y|-1 \leqslant\left|y_{i_{0}}\right| \leqslant|y|+1,|x|-1 \leqslant\left|x_{i_{0}}\right| \leqslant|x|+1$. Утверждение доказано.

ЗАмЕчАнИЕ П.1. В условиях утверждения П.1 существуют постоянные $d_{3}, Q_{3}$ и $a_{3}$ такие, что

$$
\sum_{\beta \in I} \lambda_{\beta}\left|\chi_{\beta}(z)\right|^{2} \geqslant \frac{d_{3}}{(1+|x|)^{Q_{3}}} e^{-a_{3}|y|}, \quad z \in \mathbb{C}
$$

Действительно, пользуясь неравенством Коши-Буняковского, имеем

$$
\sum_{\beta \in I} \lambda_{\beta} \sum_{\beta \in I} \lambda_{\beta}\left|\chi_{\beta}(z)\right|^{2} \geqslant\left(\sum_{\beta \in I} \lambda_{\beta}\left|\chi_{\beta}(z)\right|\right)^{2} \geqslant C \frac{e^{-2 a_{2}|y|}}{(1+|x|)^{2 Q_{2}}} .
$$

ЗАМЕЧАнИЕ П.2. В условиях утверждения П.1, не нарушая общности, можно считать, что $\sum_{\beta \in I} \lambda_{\beta}=1$.

ДокАЗАТЕЛЬСТво ЛЕммы П.1. Согласно уже доказанному сушествуют счетное множество $I \subset \Omega$, числа $\lambda_{\beta}>0, \beta \in I, \sum_{\beta \in I} \lambda_{\beta}=1$, и выполнена оценка (П.11). Рассмотрим семейство функций

$$
\psi_{\beta}(z, \bar{z})=\frac{\bar{\chi}_{\beta}(z)}{\sum_{\beta \in I}\left|\chi_{\beta}(z)\right|^{2} \lambda_{\beta}}, \quad \beta \in I .
$$

Очевидно,

$$
\sum_{\beta \in I} \chi_{\beta}(z) \psi_{\beta}(z, \bar{z}) \lambda_{\beta} \equiv 1
$$

Заметим, что

$$
\left|\left(\frac{\partial}{\partial z}\right)^{i}\left(\frac{\partial}{\partial \bar{z}}\right)^{j} \psi_{\beta}(z, \bar{z})\right| \leqslant A_{i, j} e^{a_{i, j}|y|}(1+|x|)^{Q_{i, j}}, \quad i, j=0,1, \ldots
$$


где $A_{i, j}, a_{i, j}$ и $Q_{i, j}$ не зависят от $\beta \in I$.

Рассмотрим множество функций $G_{\alpha \beta}(z, \bar{z})=\psi_{\alpha}(z, \bar{z}) \frac{\partial}{\partial \bar{z}} \psi_{\beta}(z, \bar{z}), \alpha, \beta \in I$. Нетрудно видеть, что $G_{\alpha \beta}(z, \bar{z})$ удовлетворяют оценкам (П.14). Поэтому существуют постоянные $A^{*}, a^{*}$ и $Q^{*}$ такие, что

$$
\ln \left[\left(1+|z|^{2}\right)^{2}\left|G_{\alpha \beta}(z, \bar{z})\right|^{2}\right] \leqslant A^{*}+a^{*}|y|+Q^{*} \ln \left(1+|z|^{2}\right), \quad z \in \mathbb{C} .
$$

Функция $\gamma(x, y)=A^{*}+a^{*}|y|+Q^{*} \ln \left(1+|x|^{2}+|y|^{2}\right)$ субгармоническая, и существует постоянная $C$, не зависящая от $\alpha$ и $\beta \in I$, такая, что

$$
\left\|G_{\alpha \beta}(z, \bar{z})\right\|_{L^{2}(\mathbb{C}, \gamma)}=\int\left|G_{\alpha \beta}(z, \bar{z})\right|^{2} e^{-\gamma(x, y)} d x d y \leqslant C .
$$

Согласно [6, теорема 4.4.2] существует обобщенное решение $\bar{\partial}$ задачи

$$
\frac{\partial}{\partial \bar{z}} b_{\alpha \beta}(z, \bar{z})=G_{\alpha \beta}(z, \bar{z}), \quad \alpha, \beta \in I, \quad z \in \mathbb{C},
$$

такое, что $\left\|\left(1+|z|^{2}\right)^{-1} b_{\alpha \beta}(z, \bar{z})\right\|_{L^{2}(\mathbb{C}, \gamma)}$ не превосходит некоторой постоянной, которая не зависит от $\alpha$ и $\beta$. Отсюда получаем

$$
\left\|b_{\alpha \beta}(z, \bar{z})\right\|_{L^{2}\left(\mathbb{C}, \gamma_{1}\right)}=\int\left|b_{\alpha \beta}(z, \bar{z})\right|^{2} e^{-\gamma_{1}(x, y)} d x d y \leqslant \text { const }
$$

где $\gamma_{1}(x, y)=\gamma(x, y)+2 \ln \left(1+|x|^{2}+|y|^{2}\right)$. Полагаем теперь

$$
g_{\beta}(z, \bar{z})=\psi_{\beta}(z, \bar{z})+\sum_{\alpha \in I}\left[b_{\beta \alpha}(z, \bar{z})-b_{\alpha \beta}(z, \bar{z})\right] \chi_{\alpha}(z) \lambda_{\alpha}, \quad z \in \mathbb{C} .
$$

Отметим, что из оценок (П.1) и (П.18) следует сходимость ряда (П.19) в пространстве $L^{2}\left(\mathbb{C}, \gamma_{2}\right)$, где $\gamma_{2}(x, y)=\gamma_{1}(x, y)+2 a|y|+2 Q \ln \left(1+|x|^{2}+|y|^{2}\right)$. Действительно, это легко вытекает из оценки

$$
\begin{gathered}
\left|\sum_{\alpha \in I}\left[b_{\beta \alpha}(z, \bar{z})-b_{\alpha \beta}(z, \bar{z})\right] \chi_{\alpha}(z) \lambda_{\alpha}\right| \leqslant \sup _{\alpha \in I}\left|\chi_{\alpha}(z)\right| \sum_{\alpha \in I}\left|b_{\beta \alpha}(z, \bar{z})-b_{\alpha \beta}(z, \bar{z})\right| \lambda_{\alpha} \\
\leqslant A e^{a|y|}(1+|x|)^{Q} \sum_{\alpha \in I}\left|b_{\beta \alpha}(z, \bar{z})-b_{\alpha \beta}(z, \bar{z})\right| \lambda_{\alpha} .
\end{gathered}
$$

Вычислим обобщенную производную по $\bar{z}$ от $g_{\beta}(z, \bar{z})$. Так как ряд (П.19) сходится в $L^{2}\left(\mathbb{C}, \gamma_{2}\right)$, то он сходится и в $\mathscr{D}^{\prime}$, а потому его можно дифференцировать почленно. Имеем

$$
\begin{aligned}
\frac{\partial}{\partial \bar{z}} g_{\beta}(z, \bar{z})= & \frac{\partial}{\partial \bar{z}} \psi_{\beta}(z, \bar{z})+\frac{\partial}{\partial \bar{z}} \sum_{\alpha \in I}\left[b_{\beta \alpha}(z, \bar{z})-b_{\alpha \beta}(z, \bar{z})\right] \chi_{\alpha}(z) \lambda_{\alpha} \\
= & \frac{\partial}{\partial \bar{z}} \psi_{\beta}(z, \bar{z})+\sum_{\alpha \in I} \frac{\partial b_{\beta \alpha}(z, \bar{z})}{\partial \bar{z}} \chi_{\alpha}(z) \lambda_{\alpha}-\sum_{\alpha \in I} \frac{\partial b_{\alpha \beta}(z, \bar{z})}{\partial \bar{z}} \chi_{\alpha}(z) \lambda_{\alpha} \\
= & \frac{\partial}{\partial \bar{z}} \psi_{\beta}(z, \bar{z})+\sum_{\alpha \in I} \lambda_{\alpha} \chi_{\alpha}(z)\left[G_{\beta \alpha}(z, \bar{z})-G_{\alpha \beta}(z, \bar{z})\right] \\
= & \frac{\partial}{\partial \bar{z}} \psi_{\beta}(z, \bar{z})+\psi_{\beta}(z, \bar{z}) \frac{\partial}{\partial \bar{z}} \sum_{\alpha \in I} \psi_{\alpha}(z, \bar{z}) \chi_{\alpha}(z) \lambda_{\alpha} \\
& -\left(\frac{\partial}{\partial \bar{z}} \psi_{\beta}(z, \bar{z})\right) \sum_{\alpha \in I} \psi_{\alpha}(z, \bar{z}) \chi_{\alpha}(z) \lambda_{\alpha}=0
\end{aligned}
$$


Мы учли (П.13) и аналитичность функций $\chi_{\alpha}(z)$. Таким образом, $g_{\beta}(z, \bar{z})=g_{\beta}(z)$ является целой аналитической функцией, причем $g_{\beta}(z) \in L^{2}\left(\mathbb{C}, \gamma_{2}\right)$.

Покажем, что для $g_{\beta}(z)$ выполнена оценка (П.3). Пусть $\chi_{z_{0}}(z)$ - характеристическая функция единичного круга с центром в точке $z_{0}$. Имеем

$$
\begin{aligned}
\left|g_{\beta}\left(z_{0}\right)\right| & =\left|\int g_{\beta}(z) \chi_{z_{0}}(z) d z d \bar{z}\right| \leqslant\left|\int g_{\beta}(z) e^{-\frac{1}{2} \gamma_{2}(z, \bar{z})} \chi_{z_{0}}(z) e^{\frac{1}{2} \gamma_{2}(z, \bar{z})} d z d \bar{z}\right| \\
& \leqslant \int\left|g_{\beta}(z)\right|^{2} e^{-\gamma_{2}(z, \bar{z})} d x d y \int_{\left|z-z_{0}\right|<1} e^{\gamma_{2}(z, \bar{z})} d x d y \\
& \leqslant C e^{\gamma_{2}\left(z_{0}, \bar{z}\right)} \leqslant A_{1} e^{a_{1}\left|y_{0}\right|}\left(1+\left|z_{0}\right|\right)^{Q_{1}}
\end{aligned}
$$

с некоторыми постоянными $a_{1}, A_{1}$ и $Q_{1}$.

Проверим, наконец, условие (П.4). Учитывая (П.13), имеем

$$
\begin{aligned}
\sum_{\beta \in I} \lambda_{\beta} g_{\beta}(z) \chi_{\beta}(z)= & \sum_{\beta \in I} \lambda_{\beta} \chi_{\beta}(z) \psi_{\beta}(z, \bar{z}) \\
& +\sum_{\beta \in I} \sum_{\alpha \in I} \lambda_{\beta} \lambda_{\alpha} \chi_{b}(z) \chi_{\alpha}(z)\left[b_{\beta \alpha}(z, \bar{z})-b_{\alpha \beta}(z, \bar{z})\right] \\
= & \sum_{\beta \in I} \lambda_{\beta} \chi_{\beta}(z) \psi_{\beta}(z, \bar{z})=1 .
\end{aligned}
$$

Лемма доказана.

ЛЕмма П.2 (о короне для пространства $\widetilde{E^{\uparrow}}$ ). Пусть $\Omega$ - произвольное множество индексов и задано семейство функиий $\left\{\chi_{\beta}(z) \in A_{L}\left(\Pi_{-a}^{a}\right), \beta \in \Omega\right\}$ для некоторого L, причем

$$
\sup _{\beta \in \Omega}\left\|\chi_{\beta}(z)\right\|_{A_{L}\left(\Pi_{-a}^{a}\right)}<\infty
$$

существуют постоянные $C$ и т такие, что

$$
\sup _{\beta \in \Omega}\left|\chi_{\beta}(z)\right| \geqslant \frac{C}{1+|z|^{m}}, \quad z \in \Pi_{-a}^{a}
$$

Тогда для любого $\varepsilon>0$ существуют счетное множество индексов $I \subset \Omega$, числа $\lambda_{\beta}>0, \beta \in I, \quad \sum_{\beta \in I} \lambda_{\beta}=1$, число $Q$ и семейство функиий

$$
\left\{g_{\beta}(z) \in A_{Q}\left(\Pi_{-a+\varepsilon}^{a-\varepsilon}\right), \beta \in I\right\}, \quad \sup _{\beta \in I}\left\|g_{\beta}(z)\right\|_{A_{Q}\left(\Pi_{-a+\varepsilon}^{a-\varepsilon}\right)}<\infty
$$

такие, что

$$
\sum_{\beta \in I} \lambda_{\beta} g_{\beta}(z) \chi_{\beta}(z)=1, \quad z \in \Pi_{-a+\varepsilon}^{a-\varepsilon} .
$$

Доказательство этой леммы содержится в работе [1, леммы П.0 и П.1]. 
ЛЕмма П.3 (о короне для пространства $\left.\widetilde{E^{\downarrow}}\right)$. Пусть $\Omega-$ произвольное множество индексов $и\left\{\chi_{\beta}(z), \beta \in \Omega\right\}$ - семейство иелых функиий таких, что для любого а $>0$ существуют числа $q, Q, c, C$ такие, что

$$
\begin{gathered}
\left|\chi_{\beta}(z)\right| \leqslant C(1+|z|)^{Q}, \quad z \in \Pi_{-a}^{a}, \quad \beta \in \Omega, \\
\sup _{\beta \in \Omega}\left|\chi_{\beta}(z)\right| \geqslant \frac{c}{1+|z|^{q}}, \quad z \in \Pi_{-a}^{a} .
\end{gathered}
$$

Тогда существуют счетное множество индексов $I \subset \Omega$, числа $\lambda_{\beta}>0, \beta \in I$, $\sum_{\beta \in I} \lambda_{\beta}=1$, и семейство иельх функиий $\left\{g_{\beta}(z), \beta \in I\right\}$ таких, что для любого а $>0$ найдутся числа $C_{1}$ и $Q_{1}$ такие, что

$$
\left|g_{\beta}(z)\right| \leqslant C_{1}(1+|z|)^{Q_{1}}, \quad z \in \Pi_{-a}^{a},
$$

причем

$$
\sum_{\beta \in I} \lambda_{\beta} g_{\beta}(z) \chi_{\beta}(z)=1, \quad z \in \mathbb{C}
$$

\section{Докажем сначала следующее}

УТВЕРЖДЕНИЕ П.2. Пусть $\Omega$ - произвольное множество индексов и задано семейство чельх аналитических функиий $\left\{\chi_{\beta}(z), z \in \mathbb{C}, \beta \in \Omega\right\}$, удовлетворяющих оченкам (П.24) и (П.25). Тогда существуют счетное множество $I \subset \Omega$, числа $\lambda_{\beta}>0, \beta \in I, \quad \sum_{\beta \in I} \lambda_{\beta}=1$, такие, что для любого а $>0$ найдутся числа $c_{*} u q_{*}$ такие, что

$$
\sum_{\beta \in I} \lambda_{\beta}\left|\chi_{\beta}(z)\right|^{2} \geqslant \frac{c_{*}}{(1+|z|)^{q_{*}}}, \quad z \in \Pi_{-a}^{a}
$$

Действительно, зададим последовательность $a_{j} \rightarrow+\infty$ при $j \rightarrow \infty$. Для полосы $\Pi_{-a_{j}}^{a_{j}}$ согласно $\left[1\right.$, лемма П.0 (и замечание $1 \mathrm{k}$ ней)] найдутся числа $\mu_{i}^{j}>0$, $i=1,2, \ldots, \sum_{i} \mu_{i}^{j}=1$, и функции $\chi_{i}^{j}(z)$ заданного семейства $\left\{\chi_{\beta}(z)\right\}$ такие, что $\sum_{i} \mu_{i}^{j}\left|\chi_{i}^{j}(z)\right|^{2} \geqslant \frac{c^{j}}{(1+|z|)^{j}}$ при $z \in \Pi_{-a_{j}}^{a_{j}}$ для некоторых $c^{j}$ и $q^{j}$. Положим $\lambda_{i, j}=\frac{1}{j^{2} \sigma} \mu_{i}^{j}$, где $\sigma=\sum_{j} \frac{1}{j^{2}}$. Отметим, что $\sum_{i, j} \lambda_{i, j}=1$. Теперь для любого $a>0$ сушествует $j_{0}$ такое, что $a_{j_{0}}>a$, и в полосе $\Pi_{-a}^{a}$ справедлива оценка

$$
\sum_{\substack{i=1 \\ j=1}}^{\infty} \lambda_{i, j}\left|\chi_{i}^{j}(z)\right|^{2} \geqslant \sum_{i=1}^{\infty} \lambda_{i, j_{0}}\left|\chi_{i}^{j_{0}}(z)\right|^{2}=\frac{1}{j^{2} \sigma} \sum_{i=1}^{\infty} \mu_{i}^{j_{0}}\left|\chi_{i}^{j_{0}}(z)\right|^{2} \geqslant \frac{1}{j_{0}^{2} \sigma} \frac{c^{j_{0}}}{(1+|z|)^{q^{j_{0}}}}
$$

Утверждение доказано. 
ДокАЗАТЕЛЬСТво ЛЕмМЫ П.3. Рассмотрим семейство функций $\left\{\psi_{\beta}(z, \bar{z}), \beta \in\right.$ $I\}$, определяемое формулой (П.12). Ясно, что для любого $a>0$ существуют числа $A_{i, j}$ и $Q_{i, j}$ такие, что

$$
\left|\left(\frac{\partial}{\partial z}\right)^{i}\left(\frac{\partial}{\partial \bar{z}}\right)^{j} \psi_{\beta}(z, \bar{z})\right| \leqslant A_{i, j}(1+|x|)^{Q_{i, j}}, \quad i, j=0,1, \ldots .
$$

Рассмотрим множество функций $G_{\alpha \beta}(z, \bar{z})=\psi_{\alpha}(z, \bar{z}) \frac{\partial}{\partial \bar{z}} \psi_{\beta}(z, \bar{z}), \alpha, \beta \in I$. Нетрудно видеть, что $G_{\alpha \beta}(z, \bar{z})$ удовлетворяют оценкам (П.28), а потому можно подобрать бесконечно дифференцируемые функции $A(y)$ и $Q(y)$ такие, чтобы выполнялась оценка

$$
\ln \left|G_{\alpha \beta}(z, \bar{z})\right|^{2} \leqslant A(y)+Q(y) \ln \left(1+x^{2}\right), \quad z \in \mathbb{C} .
$$

Увеличивая, если нужно, $A(y)$ и $Q(y)$, можно считать, что функция $\gamma(x, y)=$ $A(y)+Q(y) \ln \left(1+x^{2}\right)$ субгармоническая.

Действительно, например, вместо $Q(y)$ можно взять функцию

$$
Q_{1}(y)=\int_{0}^{y} d \tau \int_{0}^{\tau}\left|Q^{\prime \prime}(\xi)\right| d \xi+Q(0)+Q^{\prime}(0) y
$$

и вместо $A(y)$ - функцию

$$
A_{1}(y)=\int_{0}^{y} d \tau \int_{0}^{\tau}\left\{\left|A^{\prime \prime}(\xi)+2\right| Q_{1}(\xi) \mid\right\} d \xi+A(0)+A^{\prime}(0) y .
$$

Тогда $A_{1}(y) \geqslant A(y), Q_{1}(y) \geqslant Q(y), A_{1}^{\prime \prime}(y) \geqslant 0, Q_{1}^{\prime \prime}(y) \geqslant 0$ и $A_{1}^{\prime \prime}(y)>2\left|Q_{1}(y)\right|$. Откуда видно, что $\Delta\left(A_{1}(y)+Q_{1}(y) \ln \left(1+x^{2}\right)\right) \geqslant 0$. Далее доказательство проводится, как в лемме П.1.

ЛЕмма П.4 (о короне для пространства $\mathscr{S}$ ). Пусть $\Omega$ - произвольное множество индексов $и\left\{\chi_{\beta}(x) \in C^{\infty}\left(\mathbb{R}^{1}\right), \beta \in \Omega\right\}$ - семейство функций, удовлетворяющих условиям: существуют постоянные $q, c, Q_{j} u C_{j}, j=0,1,2, \ldots$, такие, что

$$
\begin{aligned}
& \left|\chi_{\beta}^{(j)}(x)\right| \leqslant C_{j}(1+|x|)^{Q_{j}}, \quad \beta \in \Omega, \\
& \sup _{\beta \in \Omega}\left|\chi_{\beta}(x)\right| \geqslant \frac{c}{1+|x|^{q}}, \quad x \in \mathbb{R}^{1} .
\end{aligned}
$$

Тогда существуют счетное множество индексов $I \subset \Omega$, числа $\lambda_{\beta}>0, \beta \in I$, $\sum_{\beta \in I} \lambda_{\beta}=1$, и семейство функиий $\left\{g_{\beta}(x) \in C^{\infty}\left(\mathbb{R}^{1}\right), \beta \in I\right\}$ такие, что для любого $j=0,1, \ldots$ найдутся числа $C_{j}^{1}$ и $Q_{j}^{1}$ такие, что

$$
\left|g_{\beta}^{(j)}(x)\right| \leqslant C_{j}^{1}(1+|x|)^{Q_{j}^{1}}, \quad \beta \in I,
$$

причем

$$
\sum_{\beta \in I} \lambda_{\beta} g_{\beta}(x) \chi_{\beta}(x)=1, \quad x \in \mathbb{R}^{1}
$$


ДоКАЗАТЕЛЬСТВО. Используя идеи доказательства утверждения П.1, легко показать, что существуют счетное множество $I$, числа $\lambda_{i}>0, i \in I$, такие, что $\sum_{i \in I} \lambda_{i}\left|\chi_{i}(x)\right|^{2} \geqslant \frac{d}{(1+|x|)^{\ell}}$ для некоторых $d$ и $\ell$.

Теперь положим

$$
g_{\beta}(x)=\frac{\bar{\chi}_{\beta}(x)}{\sum_{\beta \in I} \lambda_{\beta}\left|\chi_{\beta}(x)\right|^{2}} .
$$

Нетрудно проверить, что семейство $\left\{g_{\beta}(x), \beta \in I\right\}$ удовлетворяет требуемым условиям (П.30) и (П.31). Лемма доказана.

Лемма П.5. Пусть $\mathbb{B}$ - банахово пространство и $f(\xi) \in \mathbb{L}(\mathscr{D} \rightarrow \mathbb{B})$. Тогда для любого $а>0$ и $a_{1}>0$ существуют $C, N$ и $b$ такие, что

$$
\left\|L_{f}^{\varphi}(x, y)\right\|_{\mathbb{B}} \leqslant \frac{C}{y^{b}} \mathscr{P}_{N}[\varphi], \quad 0<y \leqslant 1, \quad|x|<a_{1}, \quad \forall \varphi \in \mathscr{D}_{a} .
$$

Пусть еще $\mathbb{J}$ - автомодельный функиионал порядка $\alpha$. Тогда для любых $а>0$ и $a_{1}>0$ существуют $C_{1}, N$ и $b_{1}$ такие, что

$$
\hat{\mathbb{J}}\left(y^{b_{1}}\left\|L_{f}^{\varphi}(x, y)\right\|_{\mathbb{B}}\right)=\mathbb{J}\left(\frac{1}{k^{b_{1}}}\left\|L_{f}^{\varphi}\left(x, \frac{1}{k}\right)\right\|_{\mathbb{B}}\right) \leqslant C_{1} \mathscr{P}_{N}[\varphi], \quad|x|<a_{1}, \quad \forall \varphi \in \mathscr{D}_{a} .
$$

ДоКАЗАТЕЛЬСТво. Пусть фиксированы произвольные $a>0$ и $a_{1}>0$. Заметим, что для любой $\varphi(\xi) \in \mathscr{D}_{a}$ множество основных функций $\left\{\frac{1}{y} \varphi\left(\frac{x-\xi}{y}\right): 0<y \leqslant 1\right.$, $\left.|x|<a_{1}\right\}$ принадлежит $\mathscr{D}_{a_{1}+a}$. В силу конечности порядка функционала $f$ на $\mathscr{D}_{a_{1}+a}$ сушествуют $c$ и $N$ такие, что

$$
\|(f(\xi), \psi(\xi))\|_{\mathbb{B}} \leqslant c \mathscr{P}_{N}[\psi] \quad \forall \psi \in \mathscr{D}_{a_{1}+a} .
$$

Отсюда при $|x|<a_{1}, 0<y \leqslant 1$ имеем

$$
\begin{aligned}
\left\|L_{f}^{\varphi}(x, y)\right\|_{\mathbb{B}} & =\left\|\left(f(\xi), \frac{1}{y} \varphi\left(\frac{x-\xi}{y}\right)\right)\right\|_{\mathbb{B}} \leqslant c \mathscr{P}_{N}\left[\frac{1}{y} \varphi\left(\frac{x-\xi}{y}\right)\right] \\
& =c \max _{0 \leqslant j \leqslant N} \sup _{\substack{|\xi|<a \\
|x|<a_{1}}}(1+|\xi|)^{N}\left|\left(\frac{d}{d \xi}\right)^{j} \frac{1}{y} \varphi\left(\frac{x-\xi}{y}\right)\right| \\
& \leqslant C_{2} \frac{1}{y^{N+1}} \mathscr{P}_{N}[\varphi(\xi)],
\end{aligned}
$$

откуда и следует (П.33). Далее, пользуясь (2.5), получаем

$$
\begin{aligned}
& \mathbb{J}\left(\frac{1}{k^{b_{1}}}\left\|L_{f}^{\varphi}\left(x, \frac{1}{k}\right)\right\|_{\mathbb{B}}\right) \leqslant C_{\varepsilon} \underset{k>1}{\operatorname{ess} \sup } \frac{1}{k^{\alpha-\varepsilon}} \frac{1}{k^{b_{1}}}\left\|L_{f}^{\varphi}\left(x, \frac{1}{k}\right)\right\|_{\mathbb{B}} \\
& \leqslant C_{3} \underset{k>1}{\operatorname{ess} \sup } k^{-b_{1}+\varepsilon-\alpha+N+1} \mathscr{P}_{N}[\varphi]<C_{1} \mathscr{P}_{N}[\varphi] \text {, }
\end{aligned}
$$

если $b_{1}>N+1-\alpha+\varepsilon$. Откуда вытекает (П.34). Лемма доказана. 
ЛЕмма П.6. Пусть $\mathbb{B}$ - банахово пространство, $\mu(k)>0$ - непрерывная функиия при $k \geqslant 1 u$

$$
H_{\mu}=\left\{\eta(k) \in C([1,+\infty) \rightarrow \mathbb{B}): \exists \lim _{k \rightarrow+\infty} \mu(k) \eta(k)\right\}
$$

- банахово пространство с нормой $\|\eta(k)\|_{H_{\mu}}=\sup _{k \geqslant 1} \mu(k)\|\eta(k)\|_{\mathbb{B}}$.

Пусть, далее, функция $f(\bar{x}, k) \in C(\Omega \times[1,+\infty) \rightarrow \mathbb{B})$, где $\Omega \subset \mathbb{R}^{n}$, такова, что в $\mathbb{B}$ существует

$$
\lim _{k \rightarrow+\infty} \mu(k) f(\bar{x}, k) \quad \text { для n.e. } \quad \bar{x} \in \Omega .
$$

Тогда $f(\bar{x}, k)$ измерима по $\bar{x} \in \Omega$ как функция со значениями в $H_{\mu}$.

ДокАЗАТЕЛЬСТво. Без ограничения обшности считаем $\mu(k) \equiv 1$, ибо отображение $\eta(k) \mapsto \mu(k) \eta(k)$ есть изоморфизм пространств $H=H_{\mu \equiv 1}$ на $H_{\mu}$. Положим $A(\bar{x})=\lim _{n \rightarrow+\infty} f(\bar{x}, n)$. Ясно, что $A(\bar{x})$ как предел последовательности непрерывных функций есть измеримая функция со значениями в $\mathbb{B}$, а стало быть, и в $H$ (каждому $\bar{x} \in \Omega$ сопостовляется постоянная по $k$ функция $A(\bar{x}) \in \mathbb{B})$. Так как $f(\bar{x}, k)=f(\bar{x}, k)-A(\bar{x})+A(\bar{x})$, то достаточно доказать измеримость $f(\bar{x}, k)-A(\bar{x})$.

Пусть $A_{n}(\bar{x})$ - последовательность непрерывных по $\bar{x} \in \Omega$ функций со значениями в $\mathbb{B}$ такая, что $A_{n}(\bar{x}) \rightarrow A(\bar{x})$ при п.в. $\bar{x} \in \Omega$. Рассмотрим последовательность

$$
\psi_{n}(\bar{x}, k)=\omega_{n}(k)\left[f(\bar{x}, k)-A_{n}(\bar{x})\right], \quad \omega_{n}(k)=\omega\left(\frac{k}{n}\right), \omega \in \mathscr{D}, \quad \omega(0)=1
$$

Нетрудно видеть, что $\psi_{n}(\bar{x}, k)$ - непрерывные функции по $\bar{x} \in \Omega$ со значениями в $H$ (ибо $f(\bar{x}, k)$ и $A_{n}(\bar{x})$ равномерно непрерывны на компактах по $(\bar{x}, k)$ ). Покажем, что

$$
\psi_{n}(\bar{x}, k) \underset{n \rightarrow+\infty}{\longrightarrow} f(\bar{x}, k)-A(\bar{x}) \text { в } H \text { при п.в. } \bar{x} \in \Omega .
$$

Имеем

$$
\begin{aligned}
& \sup _{k \geqslant 1}\left\|\psi_{n}(\bar{x}, k)-(f(\bar{x}, k)-A(\bar{x}))\right\|_{\mathbb{B}} \\
& \quad=\sup _{k \geqslant 1}\left\|\omega_{n}(k)\left[f(\bar{x}, k)-A_{n}(\bar{x})\right]-f(\bar{x}, k)+A(\bar{x})\right\|_{\mathbb{B}} \\
& \quad=\sup _{k \geqslant 1}\left\|\left(\omega_{n}(k)-1\right)[f(\bar{x}, k)-A(\bar{x})]+\omega_{n}(k)\left[A(\bar{x})-A_{n}(\bar{x})\right]\right\|_{\mathbb{B}} \\
& \left.\quad \leqslant \sup _{k \geqslant 1} \mid \omega_{n}(k)-1\right) \mid\|f(\bar{x}, k)-A(\bar{x})\|_{\mathbb{B}}+\left\|A(\bar{x})-A_{n}(\bar{x})\right\|_{\mathbb{B}} \underset{n \rightarrow+\infty}{\longrightarrow} 0
\end{aligned}
$$

при п.в. $\bar{x} \in \Omega$. 
Действительно, пусть $\varepsilon>0$. Сушествует $k_{0}$ такое, что $\|f(\bar{x}, k)-A(\bar{x})\|_{\mathbb{B}}<\varepsilon$ при $k>k_{0}$. В силу равномерной сходимости на компактах к нулю функций $\left(\omega_{n}(k)-1\right)$ существует $n_{0}$ такое, что $\left|\omega_{n}(k)-1\right| \cdot\|f(\bar{x}, k)-A(\bar{x})\|_{\mathbb{B}}<\varepsilon$ при $n>n_{0}$ и $1 \leqslant k \leqslant k_{0}$. Таким образом, первое слагаемое правой части неравенства (П.35) не превосходит $2 \varepsilon$ при $n>n_{0}$. Считая $\bar{x}$ таким, что $A_{n}(\bar{x}) \rightarrow A(\bar{x})$ при $n \rightarrow+\infty$, и увеличивая, если нужно, $n_{0}$, вьводим, что и второе слагаемое не превосходит $\varepsilon$ при $n>n_{0}$. Лемма доказана.

\section{Список литературы}

1. Дрожжинов Ю. Н., Завьялов Б. И. Теоремы тауберова типа для обобщенной мультипликативной свертки // Изв. РАН. Сер. матем. 2000. Т. 64. № 1. С. 37-94.

2. Гельфанд И. М., Шилов Г. Е. Обобщенные функции и действия над ними. М.: Физматгиз, 1959.

3. Владимиров В.С., Дрожсжинов Ю.Н., Завьялов Б.И. Многомерные тауберовы теоремы для обобщенных функций. М.: Наука, 1986.

4. Сенета Е. Правильно меняющиеся функции. М.: Наука, 1985.

5. Мазъя В. Г. Пространства С. Л. Соболева. Л.: Изд-во ЛГУ, 1985.

6. Хермандер Л. Введение в теорию функций нескольких комплексных переменных. М.: Мир, 1968.

7. Дрожжинов Ю. Н., Завьялов Б. И. Тауберова теорема типа Винера для обобщенных функций медленного роста // Матем. сб. 1998. Т. 189. № 7. С. 91-130.

8. Muramatu T. On Besov Spaces and Sobolev Spaces of Generalized Functions Defined on a General Region // Publ. Res. Inst. Math. Sci. Kyoto University. 1974. V. 9. № 2. P. 325-396.

9. Гарнет Дж. Ограниченные аналитические функции. М.: Мир, 1984.

10. Иосида К. Функциональный анализ. М.: Мир, 1967.

11. Данфорд Н., Швари, Джс. Т. Линейные операторы. Т. 1. Общая теория. М.: Мир, 1962.

12. Бесов О.В., Ильин В. П., Никольский С. М. Интегральные представления функций и теоремы вложения. М.: Наука, 1996.

13. Стейн И. Сингулярные интегралы и дифференциальные свойства функций. М.: Мир, 1973.

Математический институт им. В. А. Стеклова РАН, Москва

E-mail: drozzin@mi.ras.ru

Поступило в редакцию

30.VIII. 2001 